\title{
Specht modules and semisimplicity criteria for Brauer and Birman-Murakami-Wenzl algebras
}

\author{
John Enyang
}

Received: 26 April 2005 / Accepted: 8 January 2007 /

Published online: 7 April 2007

(C) Springer Science+Business Media, LLC 2007

\begin{abstract}
A construction of bases for cell modules of the Birman-Murakami-Wenzl (or B-M-W) algebra $B_{n}(q, r)$ by lifting bases for cell modules of $B_{n-1}(q, r)$ is given. By iterating this procedure, we produce cellular bases for B-M-W algebras on which a large Abelian subalgebra, generated by elements which generalise the Jucys-Murphy elements from the representation theory of the Iwahori-Hecke algebra of the symmetric group, acts triangularly. The triangular action of this Abelian subalgebra is used to provide explicit criteria, in terms of the defining parameters $q$ and $r$, for $\mathrm{B}-\mathrm{M}-\mathrm{W}$ algebras to be semisimple. The aforementioned constructions provide generalisations, to the algebras under consideration here, of certain results from the Specht module theory of the Iwahori-Hecke algebra of the symmetric group.
\end{abstract}

Keywords Birman-Murakami-Wenzl algebra $\cdot$ Brauer algebra $\cdot$ Specht module Cellular algebra $\cdot$ Jucys-Murphy operators

\section{Introduction}

Using a recursive procedure which lifts bases of $B_{i-1}(q, r)$ to bases for $B_{i}(q, r)$, for $i=1,2, \ldots, n$, we obtain new cellular bases (in the sense of [5]) for the B-M-W algebra $B_{n}(q, r)$, indexed by paths in an appropriate Bratteli diagram, whereby

1. each cell module for $B_{n}(q, r)$ admits a filtration by cell modules for $B_{n-1}(q, r)$, and

2. certain commuting elements in $B_{n}(q, r)$, which generalise the Jucys-Murphy elements in the Iwahori-Hecke algebra of the symmetric group, act triangularly on each cell module for the algebra $B_{n}(q, r)$.

Research supported by Japan Society for Promotion of Science.

\footnotetext{
J. Enyang

Graduate School of Mathematics, Nagoya University, Chikusa-ku, Nagoya 464-8602, Japan
} 
The triangular action of the generalised Jucys-Murphy elements, combined with the machinery of cellular algebras from [5], allows us to obtain explicit criteria, in terms of defining parameters, for any given B-M-W algebra to be semisimple. The aforementioned provide generalisations of classical results from the representation theory of the Iwahori-Hecke algebra of the symmetric group to the algebras under investigation here.

The contents of this article are presented as follows.

1. Definitions concerning partitions and tableaux, along with standard facts from the representation theory of the Iwahori-Hecke algebra of the symmetric group are stated in Sect. 2.

2. In Sect. 3, we define a generic version of the B-M-W algebras and restate in a more transparent notation the main results of [4] on cellular bases of the same algebras.

3. In Sect. 4, we state for reference some consequences following from the statements in Sect. 3 and the theory of cellular algebras given in [5].

4. In Sect. 5, an explicit description of the behaviour of the cell modules for generic $\mathrm{B}-\mathrm{M}-\mathrm{W}$ algebras under restriction is obtained.

5. In Sect. 6, the results of Sect. 5 are used to construct new bases for B-M-W algebras, indexed by pairs of paths in the Bratteli diagram associated with B-M-W algebras and generalising Murphy's construction [9] of bases for the Iwahori-Hecke algebras of the symmetric group. A demonstration of the iterative procedure is given in detail in Examples 6.2 and 6.3.

6. Certain results of R. Dipper and G. James on the Jucys-Murphy operators of the Iwahori-Hecke algebra of the symmetric group are extended to generic B-M-W algebras in Theorem 7.8 .

7. Theorems 8.2 and 8.5 use the above mentioned results to give sufficient criteria for the B-M-W algebras over a field to be semisimple.

8. Theorem 10.7 shows that the Jucys-Murphy elements act triangularly on each cell module of the Brauer algebra, while the semisimplicity criterion of Theorem 11.1 is a weak version of a result of H. Rui [11].

9. Some conjectures on the semisimplicity of the Brauer algebras are given in Sect. 12 .

The author is indebted to B. Srinivasan for guidance, to A. Ram for remarks on a previous version of this paper, and to I. Terada for discussions during the period this work was undertaken. The author is grateful to T. Shoji and H. Miyachi for comments and thanks the referees for numerous suggestions and corrections.

\section{Preliminaries}

\subsection{Combinatorics and tableaux}

Throughout, $n$ will denote a positive integer and $\mathfrak{S}_{n}$ will be the symmetric group acting on $\{1, \ldots, n\}$ on the right. For $i$ an integer, $1 \leq i<n$, let $s_{i}$ denote the transposition $(i, i+1)$. Then $\mathfrak{S}_{n}$ is generated as a Coxeter group by $s_{1}, s_{2}, \ldots, s_{n-1}$, which 
satisfy the defining relations

$$
\begin{array}{ll}
s_{i}^{2}=1 & \text { for } 1 \leq i<n ; \\
s_{i} s_{i+1} s_{i}=s_{i+1} s_{i} s_{i+1} & \text { for } 1 \leq i<n-1 ; \\
s_{i} s_{j}=s_{j} s_{i} & \text { for } 2 \leq|i-j| .
\end{array}
$$

An expression $w=s_{i_{1}} s_{i_{2}} \cdots s_{i_{k}}$ in which $k$ is minimal is called a reduced expression for $w$, and $\ell(w)=k$ is the length of $w$.

Let $f$ be an integer, $0 \leq f \leq[n / 2]$. If $n-2 f>0$, a partition of $n-2 f$ is a non-increasing sequence $\lambda=\left(\lambda_{1}, \ldots, \lambda_{k}\right)$ of integers, $\lambda_{i} \geq 0$, such that $\sum_{i=1}^{k} \lambda_{i}=$ $n-2 f$; otherwise, if $n-2 f=0$, write $\lambda=\varnothing$ for the empty partition. The fact that $\lambda$ is a partition of $n-2 f$ will be denoted by $\lambda \vdash n-2 f$. We will also write $|\lambda|=\sum_{i \geq 1} \lambda_{i}$. The integers $\left\{\lambda_{i}\right.$ : for $\left.i \geq 1\right\}$ are the parts of $\lambda$. If $\lambda$ is a partition of $n-2 f$, the Young diagram of $\lambda$ is the set

$$
[\lambda]=\left\{(i, j): \lambda_{i} \geq j \geq 1 \text { and } i \geq 1\right\} \subseteq \mathbb{N} \times \mathbb{N} .
$$

The elements of $[\lambda]$ are the nodes of $\lambda$ and more generally a node is a pair $(i, j) \in$ $\mathbb{N} \times \mathbb{N}$. The diagram $[\lambda]$ is traditionally represented as an array of boxes with $\lambda_{i}$ boxes on the $i$-th row. For example, if $\lambda=(3,2)$, then $[\lambda]=\square+\square$. Let $[\lambda]$ be the diagram of a partition. A node $(i, j)$ is an addable node of $[\lambda]$ if $(i, j) \notin[\lambda]$ and $[\mu]=[\lambda] \cup\{(i, j)\}$ is the diagram of a partition; in this case $(i, j)$ is also referred to as a removable node of $[\mu]$.

For our purposes, a dominance order on partitions is defined as follows: if $\lambda$ and $\mu$ are partitions, then $\lambda \unrhd \mu$ if either

1. $|\mu|>|\lambda|$ or

2. $|\mu|=|\lambda|$ and $\sum_{i=1}^{k} \lambda_{i} \geq \sum_{i=1}^{k} \mu_{i}$ for all $k>0$.

We will write $\lambda \triangleright \mu$ to mean that $\lambda \unrhd \mu$ and $\lambda \neq \mu$. Although the definition of the dominance order on partitions employed here differs from the conventional definition [7] of the dominance order on partitions, when restricted to the partitions of the odd integers $\{1,3, \ldots, n\}$ or to partitions of the even integers $\{0,2, \ldots, n\}$, depending as $n$ is odd or even, the order $\unrhd$ as defined above is compatible with a cellular structure of the Birman-Murakami-Wenzl and Brauer algebras, as shown in [4], [5] and [13].

Let $f$ be an integer, $0 \leq f \leq[n / 2]$, and $\lambda$ be a partition of $n-2 f$. A $\lambda$-tableau labeled by $\{2 f+1,2 f+2, \ldots, n\}$ is a bijection $\mathfrak{t}$ from the nodes of the diagram $[\lambda]$ to the integers $\{2 f+1,2 f+2, \ldots, n\}$. A given $\lambda$-tableau $\mathfrak{t}:[\lambda] \rightarrow\{2 f+1,2 f+$ $2, \ldots, n\}$ can be visualised by labeling the nodes of the diagram $[\lambda]$ with the integers $2 f+1,2 f+2, \ldots, n$. For example, if $n=10, f=2$ and $\lambda=(3,2,1)$,

$$
\mathfrak{t}=\begin{array}{|l|l|l|}
\hline 5 & 8 & 10 \\
\hline 6 & 7 & \\
\cline { 1 - 1 } 9 & \multicolumn{2}{|l}{} \\
\end{array}
$$

represents a $\lambda$-tableau. A $\lambda$-tableau $\mathfrak{t}$ labeled by $\{2 f+1,2 f+2, \ldots, n\}$ is said to be standard if

$$
\mathfrak{t}\left(i_{1}, j_{1}\right) \geq \mathfrak{t}\left(i_{2}, j_{2}\right), \quad \text { whenever } i_{1} \geq i_{2} \text { and } j_{1} \geq j_{2} .
$$


If $\lambda$ is a partition of $n-2 f$, write $\operatorname{Std}_{n}(\lambda)$ for the set of standard $\lambda$-tableaux labeled by the integers $\{2 f+1,2 f+2, \ldots, n\}$. We let $\mathfrak{t}^{\lambda}$ denote the element of $\operatorname{Std}_{n}(\lambda)$ in which $2 f+1,2 f+2, \ldots, n$ are entered in increasing order from left to right along the rows of $[\lambda]$. Thus in the above example where $n=10, f=2$ and $\lambda=(3,2,1)$,

$$
\mathfrak{t}^{\lambda}=\begin{array}{|l|l|l|}
\hline 5 & 6 & 7 \\
\hline 8 & 9 & \\
\hline 10 &
\end{array} .
$$

The tableau $\mathfrak{t}^{\lambda}$ is referred to as the superstandard tableau in $\operatorname{Std}_{n}(\lambda)$. If $\mathfrak{t} \in \operatorname{Std}_{n}(\lambda)$, we will write $\lambda=\operatorname{Shape}(\mathfrak{t})$ and, abiding by the convention used in the literature, $\operatorname{Std}(\lambda)$ will be used to denote the set of standard tableaux $t:[\lambda] \rightarrow\{1,2, \ldots,|\lambda|\}$; we will refer to elements of $\operatorname{Std}(\lambda)$ simply as standard $\lambda$-tableaux. If $\mathfrak{s} \in \operatorname{Std}_{n}(\lambda)$, we will write $\hat{\mathfrak{s}}$ for the tableau in $\operatorname{Std}(\lambda)$ which is obtained by relabelling the nodes of $\mathfrak{s}$ by the map $i \mapsto i-2 f$.

If $\mathfrak{t} \in \operatorname{Std}_{n}(\lambda)$ and $i$ is an integer $2 f<i \leq n$, define $\left.\mathfrak{t}\right|_{i}$ to be the tableau obtained by deleting each entry $k$ of $\mathfrak{t}$ with $k>i$ (compare Example 5.1 below). The set $\operatorname{Std}_{n}(\lambda)$ admits an order $\unrhd$ wherein $\mathfrak{s} \unrhd \mathfrak{t}$ if Shape $\left(\left.\mathfrak{s}\right|_{i}\right) \unrhd \operatorname{Shape}\left(\left.\mathfrak{t}\right|_{i}\right)$ for each integer $i$ with $2 f<i \leq n$. We adopt the usual convention of writing $\mathfrak{s} \triangleright \mathfrak{t}$ to mean that $\mathfrak{s} \unrhd \mathfrak{t}$ and $\mathfrak{s} \neq \mathfrak{t}$.

The subgroup $\mathfrak{S}_{n-2 f}=\left\langle s_{i}: 2 f<i<n\right\rangle \subset \mathfrak{S}_{n}$ acts on the set of $\lambda$-tableaux on the right in the usual manner, by permuting the integer labels of the nodes of $[\lambda]$. For example,

$$
\begin{array}{|l|l|l|}
\hline 5 & 6 & 7 \\
\hline 8 & 9 & (6,8)(7,10,9)=\begin{array}{|l|l|l|}
\hline 5 & 8 & 10 \\
\hline 10 &
\end{array} \quad \begin{array}{ll}
\hline 6 & 7 \\
\hline 9 &
\end{array} \\
\hline
\end{array}
$$

If $\lambda$ is a partition of $n-2 f$, then for our purposes the Young subgroup $\mathfrak{S}_{\lambda}$ is defined to be the row stabiliser of $\mathfrak{t}^{\lambda}$ in $\mathfrak{S}_{n-2 f}$. For instance, when $n=10, f=2$ and $\lambda=(3,2,1)$, as in (2.2) above, then $\mathfrak{S}_{\lambda}=\left\langle s_{5}, s_{6}, s_{8}\right\rangle$. To each $\lambda$-tableau $\mathfrak{t}$, associate a unique permutation $d(\mathfrak{t}) \in \mathfrak{S}_{n-2 f}$ by the condition $\mathfrak{t}=\mathfrak{t}^{\lambda} d(\mathfrak{t})$. If we refer to the tableau $\mathfrak{t}$ in $(2.1)$ above for instance, then $d(\mathfrak{t})=(6,8)(7,10,9)$ by $(2.3)$.

\subsection{The Iwahori-Hecke algebra of the symmetric group}

For the purposes of this section, let $R$ denote an integral domain and $q$ be a unit in $R$. The Iwahori-Hecke algebra (over $R$ ) of the symmetric group is the unital associative $R$-algebra $\mathscr{H}_{n}\left(q^{2}\right)$ with generators $X_{1}, X_{2}, \ldots, X_{n-1}$, which satisfy the defining relations

$$
\begin{array}{ll}
\left(X_{i}-q\right)\left(X_{i}+q^{-1}\right)=0 & \text { for } 1 \leq i<n ; \\
X_{i} X_{i+1} X_{i}=X_{i+1} X_{i} X_{i+1} & \text { for } 1 \leq i<n-1 ; \\
X_{i} X_{j}=X_{j} X_{i} & \text { for } 2 \leq|i-j| .
\end{array}
$$

If $w \in \mathfrak{S}_{n}$ and $s_{i_{1}} s_{i_{2}} \cdots s_{i_{k}}$ is a reduced expression for $w$, then

$$
X_{w}=X_{i_{1}} X_{i_{2}} \cdots X_{i_{k}}
$$


is a well defined element of $\mathscr{H}_{n}\left(q^{2}\right)$ and the set $\left\{X_{w}: w \in \mathfrak{S}_{n}\right\}$ freely generates $\mathscr{H}_{n}\left(q^{2}\right)$ as an $R$-module (Theorems 1.8 and 1.13 of [8]).

Below we state for later reference standard facts from the representation theory of the Iwahori-Hecke algebra of the symmetric group, of which details can be found in [8] or [9]. If $\mu$ is a partition of $n$, define the element

$$
c_{\mu}=\sum_{w \in \mathfrak{S}_{\mu}} q^{l(w)} X_{w} .
$$

In this section, let $*$ denote the algebra anti-involution of $\mathscr{H}_{n}\left(q^{2}\right)$ mapping $X_{w} \mapsto$ $X_{w^{-1}}$. If $\lambda$ is a partition of $n, \check{\mathscr{H}}_{n}^{\lambda}$ is defined to be the two-sided ideal in $\mathscr{H}_{n}\left(q^{2}\right)$ generated by

$$
\left\{c_{\mathfrak{u v}}=X_{d(\mathfrak{u})}^{*} c_{\mu} X_{d(\mathfrak{v})}: \mathfrak{u}, \mathfrak{v} \in \operatorname{Std}(\mu), \text { where } \mu \triangleright \lambda\right\}
$$

The next statement is due to E. Murphy in [9].

Theorem 2.1 The Iwahori-Hecke algebra $\mathscr{H}_{n}\left(q^{2}\right)$ is free as an $R$-module with basis

$$
\mathcal{M}=\left\{\begin{array}{l|l}
c_{\mathfrak{u} \mathfrak{v}}=X_{d(\mathfrak{u})}^{*} c_{\lambda} X_{d(\mathfrak{v})} & \begin{array}{c}
\text { for } \mathfrak{u}, \mathfrak{v} \in \operatorname{Std}(\lambda) \text { and } \\
\lambda \text { a partition of } n
\end{array}
\end{array}\right\} .
$$

Moreover, the following statements hold.

1. The $R$-linear anti-involution $*$ satisfies $*: c_{\mathfrak{s t}} \mapsto c_{\mathfrak{t s}}$ for all $\mathfrak{s}, \mathfrak{t} \in \operatorname{Std}(\lambda)$.

2. Suppose that $h \in \mathscr{H}_{n}\left(q^{2}\right)$, and that $\mathfrak{s}$ is a standard $\lambda$-tableau. Then there exist $a_{\mathfrak{u}} \in R$, for $\mathfrak{u} \in \operatorname{Std}(\lambda)$, such that for all $\mathfrak{v} \in \operatorname{Std}(\lambda)$,

$$
c_{\mathfrak{v} \mathfrak{s}} h \equiv \sum_{\mathfrak{u} \in \operatorname{Std}(\lambda)} a_{\mathfrak{u}} c_{\mathfrak{v} \mathfrak{u}} \bmod \check{\mathscr{H}}_{n}^{\lambda}
$$

The basis $\mathcal{M}$ is cellular in the sense of [5]. If $\lambda$ is a partition of $n$, the cell (or Specht) module $C^{\lambda}$ for $\mathscr{H}_{n}\left(q^{2}\right)$ is the $R$-module freely generated by

$$
\left\{c_{\mathfrak{s}}=c_{\lambda} X_{d(\mathfrak{s})}+\check{\mathscr{H}}_{n}^{\lambda}: \mathfrak{s} \in \operatorname{Std}(\lambda)\right\}
$$

and given the right $\mathscr{H}_{n}\left(q^{2}\right)$-action

$$
c_{\mathfrak{s}} h=\sum_{\mathfrak{u} \in \operatorname{Std}(\lambda)} a_{\mathfrak{u}} c_{\mathfrak{u}}, \quad \text { for } h \in \mathcal{H}_{n}\left(q^{2}\right),
$$

where the coefficients $a_{\mathfrak{u}} \in R$, for $\mathfrak{u} \in \operatorname{Std}(\lambda)$, are determined by the expression (2.4). The basis (2.5) is referred to as the Murphy basis for $C^{\lambda}$ and $\mathcal{M}$ is the Murphy basis for $\mathscr{H}_{n}\left(q^{2}\right)$.

Remark 2.1 The $\mathcal{H}_{n}\left(q^{2}\right)$-module $C^{\lambda}$ is the contragradient dual of the Specht module defined in [2]. 
Let $\lambda$ and $\mu$ be partitions of $n$. A $\lambda$-tableau of type $\mu$ is a map $\mathrm{T}:[\lambda] \rightarrow$ $\{1,2, \ldots, d\}$ such that $\mu_{i}=|\{y \in[\lambda]: \mathrm{T}(y)=i\}|$ for $i \geq 1$. A $\lambda$-tableau $\mathrm{T}$ of type $\mu$ is said to be semistandard if (i) the entries in each row of $T$ are non-decreasing, and (ii) the entries in each column of $\mathrm{T}$ are strictly increasing. If $\mu$ is a partition, the semistandard tableau $\mathrm{T}^{\mu}$ is defined to be the tableau of type $\mu$ with $\mathrm{T}^{\mu}(i, j)=i$ for $(i, j) \in[\mu]$.

Example 2.1 Let $\mu=(3,2,1)$. Then the semistandard tableaux of type $\mu$ are

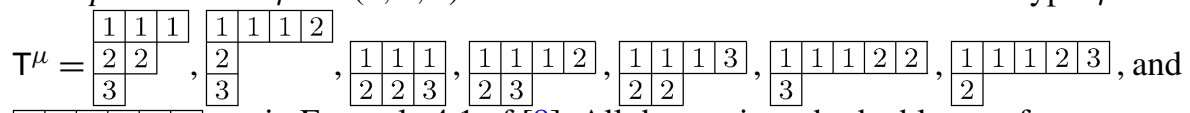
\begin{tabular}{|l|l|l|l|l|l|l}
\hline 1 & 1 & 1 & 2 & 2 & 3
\end{tabular} , as in Example 4.1 of [8]. All the semistandard tableaux of type $\mu$ are obtainable from $\mathrm{T}^{\mu}$ by "moving nodes up" in $\mathrm{T}^{\mu}$.

If $\lambda$ and $\mu$ are partitions of $n$, the set of semistandard $\lambda$-tableaux of type $\mu$ will be denoted by $\mathcal{T}_{0}(\lambda, \mu)$. Further, given a $\lambda$-tableau $\mathfrak{t}$ and a partition $\mu$ of $n$, then $\mu(\mathfrak{t})$ is defined to be the $\lambda$-tableau of type $\mu$ obtained from $\mathfrak{t}$ by replacing each entry $i$ in $\mathfrak{t}$ with $k$ if $i$ appears in the $k$-th row of the superstandard tableau $\mathfrak{t}^{\mu} \in \operatorname{Std}(\mu)$.

Example 2.2 Let $n=7$, and $\mu=(3,2,1,1)$, so that $\mathfrak{t}^{\mu}=$\begin{tabular}{|l|l|l|}
\hline 1 & 2 & 3 \\
\hline 4 & 5 & \\
\cline { 1 - 1 } &
\end{tabular}$\quad$. If $v=(4,3)$ and $\mathfrak{t}=$\begin{tabular}{|l|l|l|l}
\hline 1 & 2 & 3 & 7 \\
\hline 4 & 5 & 6
\end{tabular}, then $\mu(\mathfrak{t})=$\begin{tabular}{|l|l|l|l|}
\hline 1 & 1 & 1 & 4 \\
\hline 2 & 2 & 3
\end{tabular}.

Let $\mu$ and $\nu$ be partitions of $n$. If $\mathrm{S}$ is a semistandard $\nu$-tableau of type $\mu$, and $\mathfrak{t}$ is a standard $\nu$-tableau, define in $\mathscr{H}_{n}\left(q^{2}\right)$ the element

$$
c_{\mathrm{St}}=\sum_{\substack{\mathfrak{s} \in \operatorname{Std}(\nu) \\ \mu(\mathfrak{s})=\mathrm{S}}} q^{\ell(d(\mathfrak{s}))} c_{\mathfrak{s t}}
$$

Given a partition $\mu$ of $n$, let $M^{\mu}$ be the right $\mathscr{H}_{n}\left(q^{2}\right)$-module generated by $c_{\mu}$. The next statement is a special instance of a theorem of E. Murphy (Theorem 4.9 of [8]).

Theorem 2.2 Let $\mu$ be a partition of $n$. Then the collection

$$
\left\{c_{\mathrm{S} \mathfrak{t}}: \mathrm{S} \in \mathcal{T}_{0}(v, \mu), \mathfrak{t} \in \mathrm{Std}(v), \text { for } v \text { a partition of } n\right\}
$$

freely generates $M^{\mu}$ as an $R-$ module.

If $\mu$ and $\lambda$ are partitions of $n-1$ and $n$ respectively, for the purposes of the present Sect. 2.2, we write $\mu \rightarrow \lambda$ to mean that the diagram $[\lambda]$ is obtained by adding a node to the diagram $[\mu]$, as exemplified by the truncated Bratteli diagram associated with 
$\mathscr{H}_{n}\left(q^{2}\right)$ displayed in (2.7) below (Sect. 4 of [6]).

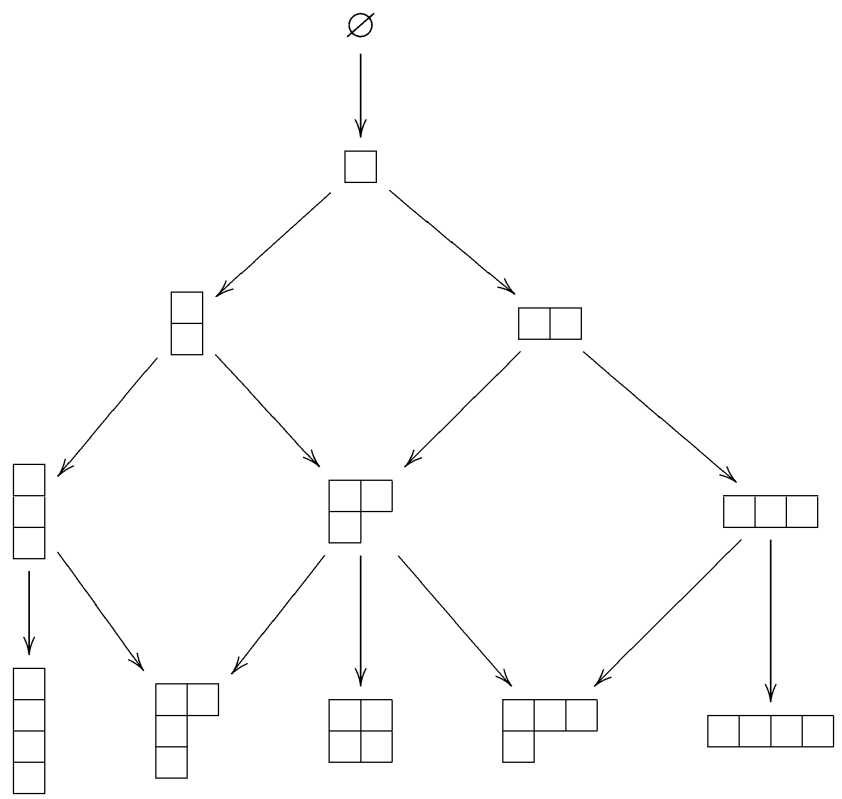

If $\lambda$ is a partition of $n$ then, as in [6], define a path of shape $\lambda$ in the Bratteli diagram associated with $\mathscr{H}_{n}\left(q^{2}\right)$ to be a sequence of partitions

$$
\left(\lambda^{(0)}, \lambda^{(1)}, \ldots, \lambda^{(n)}\right)
$$

satisfying the conditions that $\lambda^{(0)}=\varnothing$ is the empty partition, $\lambda^{(n)}=\lambda$, and $\lambda^{(i-1)} \rightarrow$ $\lambda^{(i)}$, for $1 \leq i \leq n$. As observed in Sect. 4 of [6], there is a natural correspondence between the paths in the Bratteli diagram associated with $\mathscr{H}_{n}\left(q^{2}\right)$ and the elements of $\operatorname{Std}(\lambda)$ whereby $\mathfrak{t} \mapsto\left(\lambda^{(0)}, \lambda^{(1)}, \ldots, \lambda^{(n)}\right)$ and $\lambda^{(i)}=\operatorname{Shape}\left(\left.\mathfrak{t}\right|_{i}\right)$ for $1 \leq i \leq n$.

Example 2.3 Let $n=6$ and $\lambda=(3,2,1)$. Then the identification of standard $\lambda$ tableau with paths of shape $\lambda$ in the Bratteli diagram associated with $\mathscr{H}_{n}\left(q^{2}\right)$ maps

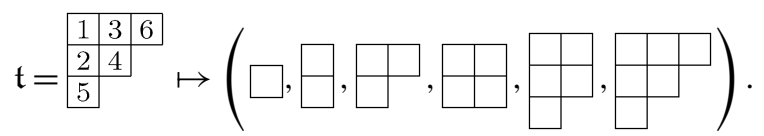

Taking advantage of the bijection between the standard $\lambda$-tableaux and the paths of shape $\lambda$ in the Bratteli diagram of $\mathscr{H}_{n}\left(q^{2}\right)$, we will have occasion to write

$$
\mathfrak{t}=\left(\lambda^{(0)}, \lambda^{(1)}, \ldots, \lambda^{(n)}\right)
$$

explicitly identifying each standard $\lambda$-tableau $\mathfrak{t}$ with a path of shape $\lambda$ in the Bratteli diagram. 
For each integer $i$ with $1 \leq i \leq n$, consider $\mathscr{H}_{i}\left(q^{2}\right)$ as the subalgebra of $\mathscr{H}_{n}\left(q^{2}\right)$ generated by the elements $X_{1}, X_{2}, \ldots, X_{i-1}$, thereby obtaining the tower of algebras

$$
R=\mathscr{H}_{1}\left(q^{2}\right) \subseteq \mathscr{H}_{2}\left(q^{2}\right) \subseteq \cdots \subseteq \mathscr{H}_{n}\left(q^{2}\right)
$$

Given a right $\mathscr{H}_{n}\left(q^{2}\right)$-module $V$, write $\operatorname{Res}(V)$ for the restriction of $V$ to $\mathscr{H}_{n-1}\left(q^{2}\right)$ by the identifications (2.8). Lemma 2.3 below, which is a consequence of Theorem 7.2 of [9], shows that the Bratteli diagram associated with $\mathscr{H}_{n}\left(q^{2}\right)$ describes the behaviour of the cell modules for $\mathscr{H}_{n}\left(q^{2}\right)$ under restriction to $\mathscr{H}_{n-1}\left(q^{2}\right)$.

Lemma 2.3 Let $\lambda$ be a partition of $n$. For each partition $\mu$ of $n-1$ with $\mu \rightarrow \lambda$, let $A^{\mu}$ denote the $R$-submodule of $C^{\lambda}$ freely generated by

$$
\left\{c_{\mathfrak{v}}: \mathfrak{v} \in \operatorname{Std}(\lambda) \text { and } \operatorname{Shape}\left(\left.\mathfrak{v}\right|_{n-1}\right) \unrhd \mu\right\}
$$

and write $\check{A}^{\mu}$ for the $R$-submodule of $S^{\lambda}$ freely generated by

$$
\left\{c_{\mathfrak{v}}: \mathfrak{v} \in \operatorname{Std}(\lambda) \text { and } \operatorname{Shape}\left(\left.\mathfrak{v}\right|_{n-1}\right) \triangleright \mu\right\}
$$

If $\mathfrak{v} \in \operatorname{Std}_{n}(\lambda)$ and $\left.\mathfrak{v}\right|_{n-1}=\mathfrak{t}^{\mu}$, then the $R$-linear map determined on generators by

$$
c_{\mathfrak{v}} X_{d(\mathfrak{u})}+\check{A}^{\mu} \mapsto c_{\mathfrak{u}}, \quad \text { for } \mathfrak{u} \in \operatorname{Std}(\mu),
$$

is an isomorphism $A^{\mu} / \check{A}^{\mu} \cong C^{\mu}$ of $\mathscr{H}_{n-1}\left(q^{2}\right)$-modules.

The Jucys-Murphy operators $\tilde{D}_{i}$ in $\mathscr{H}_{n}\left(q^{2}\right)$ are usually defined (Sect. 3 of [8]) by $\tilde{D}_{1}=0$ and

$$
\tilde{D}_{i}=\sum_{k=1}^{i-1} X_{(k, i)}, \quad \text { for } i=1, \ldots, n .
$$

As per an exercise in [8], we define $D_{1}=1$ and set $D_{i}=X_{i-1} D_{i-1} X_{i-1}$. Since $D_{i}=1+\left(q-q^{-1}\right) \tilde{D}_{i}$, and the $\tilde{D}_{i}$ can be cumbersome, we work with the $D_{i}$ rather than the $\tilde{D}_{i}$. We also refer to the $D_{i}$ as Jucys-Murphy elements; this should cause no confusion. The following proposition is well known.

Proposition 2.4 Let $i$ and $k$ be integers, $1 \leq i<n$ and $1 \leq k \leq n$.

1. $X_{i}$ and $D_{k}$ commute if $i \neq k-1, k$.

2. $D_{i}$ and $D_{k}$ commute.

3. $X_{i}$ commutes with $D_{i} D_{i+1}$ and $D_{i}+D_{i+1}$.

Let $\mathfrak{t}=\left(\lambda^{(0)}, \lambda^{(1)}, \ldots, \lambda^{(n)}\right)$ be a standard $\lambda$-tableau identified with the corresponding path in the Bratteli diagram of $\mathscr{H}_{n}\left(q^{2}\right)$. For each integer $k$ with $1 \leq k \leq n$, define

$$
P_{\mathfrak{t}}(k)=q^{2(j-i)} \quad \text { where }\left[\lambda^{(k)}\right]=\left[\lambda^{(k-1)}\right] \cup\{(i, j)\} .
$$

The next statement is due to R. Dipper and G. James (Theorem 3.32 of [8]). 
Theorem 2.5 Suppose that $\lambda$ is a partition of $n$ and let $\mathfrak{s}$ be a standard $\lambda$-tableau. If $k$ is an integer, $1 \leq k \leq n$, then there exist $a_{\mathfrak{v}} \in R$, for $\mathfrak{v} \triangleright \mathfrak{s}$, such that

$$
c_{\mathfrak{s}} D_{k}=P_{\mathfrak{s}}(k) c_{\mathfrak{s}}+\sum_{\substack{\mathfrak{v} \in \operatorname{Std}(\lambda) \\ \mathfrak{v} \triangleright \mathfrak{s}}} a_{\mathfrak{v}} c_{\mathfrak{v}} .
$$

One objective at hand is to provide an extension of Lemma 2.3 and Theorem 2.5 to the Brauer and Birman-Murakami-Wenzl algebras.

\section{The Birman-Murakami-Wenzl algebras}

Let $q, r$ be indeterminates over $\mathbb{Z}$ and $R=\mathbb{Z}\left[q^{ \pm 1}, r^{ \pm 1},\left(q-q^{-1}\right)^{-1}\right]$. The BirmanMurakami-Wenzl algebra $B_{n}(q, r)$ over $R$ is the unital associative $R$-algebra generated by the elements $T_{1}, T_{2}, \ldots, T_{n-1}$, which satisfy the defining relations

$$
\begin{array}{ll}
\left(T_{i}-q\right)\left(T_{i}+q^{-1}\right)\left(T_{i}-r^{-1}\right)=0 & \text { for } 1 \leq i<n ; \\
T_{i} T_{i+1} T_{i}=T_{i+1} T_{i} T_{i+1} & \text { for } 1 \leq i \leq n-2 ; \\
T_{i} T_{j}=T_{j} T_{i} & \text { for } 2 \leq|i-j| ; \\
E_{i} T_{i-1}^{ \pm 1} E_{i}=r^{ \pm 1} E_{i} & \text { for } 2 \leq i \leq n-1 ; \\
E_{i} T_{i+1}^{ \pm 1} E_{i}=r^{ \pm 1} E_{i} & \text { for } 1 \leq i \leq n-2 ; \\
T_{i} E_{i}=E_{i} T_{i}=r^{-1} E_{i} & \text { for } 1 \leq i \leq n-1,
\end{array}
$$

where $E_{i}$ is the element defined by the expression

$$
\left(q-q^{-1}\right)\left(1-E_{i}\right)=T_{i}-T_{i}^{-1}
$$

Writing

$$
z=\frac{(q+r)(q r-1)}{r(q+1)(q-1)},
$$

then (Sect. 3 of [12]) one derives additional relations

$$
\begin{aligned}
& E_{i}^{2}=z E_{i}, \\
& E_{i} T_{i}^{ \pm 1}=r^{\mp 1} E_{i}=T_{i}^{ \pm 1} E_{i}, \\
& T_{i}^{2}=1+\left(q-q^{-1}\right)\left(T_{i}-r^{-1} E_{i}\right) \\
& E_{i \pm 1} T_{i} T_{i \pm 1}=T_{i} T_{i \pm 1} E_{i} \\
& E_{i} T_{i \pm 1} E_{i}=r E_{i} \\
& E_{i} T_{i \pm 1}^{-1} E_{i}=r^{-1} E_{i} \\
& E_{i} E_{i \pm 1} E_{i}=E_{i} \\
& E_{i} E_{i \pm 1}=E_{i} T_{i \pm 1} T_{i}=T_{i \pm 1} T_{i} E_{i \pm 1} .
\end{aligned}
$$


If $w \in \mathfrak{S}_{n}$ is a permutation and $w=s_{i_{1}} s_{i_{2}} \cdots s_{i_{k}}$ is a reduced expression for $w$, then

$$
T_{w}=T_{i_{1}} T_{i_{2}} \cdots T_{i_{k}}
$$

is a well defined element of $B_{n}(q, r)$.

Remark 3.1 The generator $T_{i}$ above differs by a factor of $q$ from the generator used in [4] but coincides with the element $g_{i}$ of [6] and [12].

If $f$ is an integer, $0 \leq f \leq[n / 2]$, define $B_{n}^{f}$ to be the two sided ideal of $B_{n}(q, r)$ generated by the element $E_{1} E_{3} \cdots E_{2 f-1}$. Then

$$
\text { (0) } \subseteq B_{n}^{[n / 2]} \subseteq B_{n}^{[n / 2]-1} \subseteq \cdots \subseteq B_{n}^{1} \subseteq B_{n}^{0}=B_{n}(q, r)
$$

gives a filtration of $B_{n}(q, r)$. As in Theorem 4.1 of [4] (see also [13]), refining the filtration (3.2) gives the cell modules, in the sense of [5], for the algebra $B_{n}(q, r)$. If $f$ is an integer, $0 \leq f \leq[n / 2]$, and $\lambda$ is a partition of $n-2 f$, define the element

$$
x_{\lambda}=\sum_{w \in \mathfrak{S}_{\lambda}} q^{\ell(w)} T_{w},
$$

where $\mathfrak{S}_{\lambda}$ is row stabiliser in the subgroup $\left\langle s_{i}: 2 f<i<n\right\rangle$ of the superstandard tableau $\mathfrak{t}^{\lambda} \in \operatorname{Std}_{n}(\lambda)$ as defined in Sect. 2; finally define

$$
m_{\lambda}=E_{1} E_{3} \cdots E_{2 f-1} x_{\lambda}
$$

which is the analogue to the element $c_{\lambda}$ in the Iwahori-Hecke algebra of the symmetric group.

Example 3.1 Let $n=10$ and $\lambda=(3,2,1)$. From the $\lambda$-tableau displayed in (2.2) comes the subgroup $\mathfrak{S}_{\lambda}=\left\langle s_{5}, s_{6}, s_{8}\right\rangle$, and

$$
\begin{aligned}
m_{\lambda} & =E_{1} E_{3} \sum_{w \in \mathfrak{S}_{\lambda}} q^{\ell(w)} T_{w} \\
& =E_{1} E_{3}\left(1+q T_{5}\right)\left(1+q T_{6}+q^{2} T_{6} T_{5}\right)\left(1+q T_{8}\right) .
\end{aligned}
$$

If $f$ is an integer, $0 \leq f \leq[n / 2]$, define

$$
\mathbb{D}_{f, n}=\left\{v \in \mathfrak{S}_{n} \mid \begin{array}{c}
(2 i+1) v<(2 j+1) v \text { for } 0 \leq i<j<f \\
(2 i+1) v<(2 i+2) v \text { for } 0 \leq i<f \\
\text { and }(i) v<(i+1) v \text { for } 2 f<i<n
\end{array}\right\} .
$$

As shown in Sect. 3 of [4], the collection $\mathscr{D}_{f, n}$ is a complete set of right coset representatives for the subgroup $\mathfrak{B}_{f} \times \mathfrak{S}_{n-2 f}$ in $\mathfrak{S}_{n}$, where $\mathfrak{S}_{n-2 f}$ is identified with the subgroup $\left\langle s_{i}: 2 f<i<n\right\rangle$ of $\mathfrak{S}_{n}$ and $\mathfrak{B}_{0}=\langle 1\rangle, \mathfrak{B}_{1}=\left\langle s_{1}\right\rangle$ and, for $f>1$,

$$
\mathfrak{B}_{f}=\left\langle s_{2 i-1}, s_{2 i} s_{2 i+1} s_{2 i-1} s_{2 i}: 1 \leq i \leq f\right\rangle .
$$

Additionally, it is evident (Proposition 3.1 of [4]) that if $v$ is an element of $\mathscr{D}_{f, n}$, then $\ell(u v)=\ell(u)+\ell(v)$ for all $u$ in $\left\langle s_{i}: 2 f<i<n\right\rangle$. 
Remark 3.2 After fixing a choice of over and under crossings, there is a natural bijection between the coset representatives $\mathcal{D}_{f, n}$ and the $(n, n-2 f)$-dangles of Definition 3.3 of [13].

For each partition $\lambda$ of $n-2 f$, define $\mathcal{I}_{n}(\lambda)$ to be the set of ordered pairs

$$
\mathcal{I}_{n}(\lambda)=\left\{(\mathfrak{s}, v): \mathfrak{s} \in \operatorname{Std}_{n}(\lambda) \text { and } v \in \mathscr{D}_{f, n},\right\}
$$

and define $B_{n}^{\lambda}$ to be the two-sided ideal in $B_{n}(q, r)$ generated by $m_{\lambda}$ and let

$$
\check{B}_{n}^{\lambda}=\sum_{\mu \triangleright \lambda} B_{n}^{\mu}
$$

so that $B_{n}^{f+1} \subseteq \breve{B}_{n}^{\lambda}$, by the definition of the dominance order on partitions given in Sect. 2. Let $*$ be the algebra anti-involution of $B_{n}(q, r)$ which maps $T_{w} \mapsto T_{w^{-1}}$ and $E_{i} \mapsto E_{i}$.

That $B_{n}(q, r)$ is cellular in the sense of [5] was shown in [13]; the next statement which is Theorem 4.1 of [4], gives an explicit cellular basis for $B_{n}(q, r)$.

Theorem 3.1 The algebra $B_{n}(q, r)$ is freely generated as an $R$-module by the collection

$$
\left\{\begin{array}{l|l}
T_{v}^{*} T_{d(\mathfrak{s})}^{*} m_{\lambda} T_{d(\mathfrak{t})} T_{u} & \begin{array}{c}
(\mathfrak{s}, v),(\mathfrak{t}, u) \in \mathcal{I}_{n}(\lambda), \text { for } \lambda \text { a partition } \\
\text { of } n-2 f, \text { and } 0 \leq f \leq[n / 2]
\end{array}
\end{array}\right\}
$$

Moreover, the following statements hold.

1. The algebra anti-involution $*$ satisfies

$$
*: T_{v}^{*} T_{d(\mathfrak{s})}^{*} m_{\lambda} T_{d(\mathfrak{t})} T_{u} \mapsto T_{u}^{*} T_{d(\mathfrak{t})}^{*} m_{\lambda} T_{d(\mathfrak{s})} T_{v}
$$

for all $(\mathfrak{s}, v),(\mathfrak{t}, u) \in \mathcal{I}_{n}(\lambda)$.

2. Suppose that $b \in B_{n}(q, r)$ and let $f$ be an integer, $0 \leq f \leq[n / 2]$. If $\lambda$ is a partition of $n-2 f$ and $(\mathfrak{t}, u) \in \mathcal{I}_{n}(\lambda)$, then there exist $a_{(\mathfrak{u}, w)} \in R$, for $(\mathfrak{u}, w) \in \mathcal{I}_{n}(\lambda)$, such that for all $(\mathfrak{s}, v) \in \mathcal{I}_{n}(\lambda)$,

$$
T_{v}^{*} T_{d(\mathfrak{s})}^{*} m_{\lambda} T_{d(\mathfrak{t})} T_{u} b \equiv \sum_{(\mathfrak{u}, w)} a_{(\mathfrak{u}, w)} T_{v}^{*} T_{d(\mathfrak{s})}^{*} m_{\lambda} T_{d(\mathfrak{u})} T_{w} \quad \bmod \check{B}_{n}^{\lambda} .
$$

As a consequence of the above theorem, $\check{B}_{n}^{\lambda}$ is the $R$-module freely generated by the collection

$$
\left\{T_{v}^{*} T_{d(\mathfrak{s})}^{*} m_{\mu} T_{d(\mathfrak{t})} T_{u}:(\mathfrak{s}, v),(\mathfrak{t}, u) \in \mathcal{I}_{n}(\mu), \text { for } \mu \triangleright \lambda\right\}
$$

If $f$ is an integer, $0 \leq f \leq[n / 2]$, and $\lambda$ is a partition of $n-2 f$, the cell module $S^{\lambda}$ is defined to be the $R$-module freely generated by

$$
\left\{m_{\lambda} T_{d(\mathfrak{t})} T_{u}+\check{B}_{n}^{\lambda}:(\mathfrak{t}, u) \in \mathcal{I}_{n}(\lambda)\right\}
$$


and given the right $B_{n}(q, r)$ action

$$
m_{\lambda} T_{d(\mathfrak{t})} T_{u} b+\check{B}_{n}^{\lambda}=\sum_{(\mathfrak{u}, w)} a_{(\mathfrak{u}, w)} m_{\lambda} T_{d(\mathfrak{u})} T_{w}+\check{B}_{n}^{\lambda} \quad \text { for } b \in B_{n}(q, r),
$$

where the coefficients $a_{(\mathfrak{u}, w)} \in R$, for $(\mathfrak{u}, w)$ in $\mathcal{I}_{n}(\lambda)$, are determined by the expression (3.4).

Example 3.2 Let $n=6, f=1$, and $\lambda=(3,1)$. If $i, j$ are integers with $1 \leq i<j \leq n$, write $v_{i, j}=s_{2} s_{3} \cdots s_{j-1} s_{1} s_{2} \cdots s_{i-1}$, so that

$$
\mathscr{D}_{f, n}=\left\{v_{i, j}: 1 \leq i<j \leq n\right\} .
$$

Since

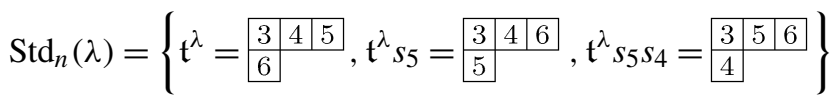

and $m_{\lambda}=E_{1}\left(1+q T_{4}\right)\left(1+q T_{3}+q^{2} T_{3} T_{4}\right)$, the basis for $S^{\lambda}$, of the form displayed in $(3.5)$, is

$$
\left\{m_{\lambda} T_{d(\mathfrak{s})} T_{v_{i, j}}+\check{B}_{n}^{\lambda}: \mathfrak{s} \in \operatorname{Std}_{n}(\lambda) \text { and } 1 \leq i<j \leq n\right\}
$$

As in Proposition 2.4 of [5], the cell module $S^{\lambda}$ for $B_{n}(q, r)$ admits a symmetric associative bilinear form $\langle\rangle:, S^{\lambda} \times S^{\lambda} \rightarrow R$ defined by

$$
\left\langle m_{\lambda} T_{d(\mathfrak{u})} T_{v}, m_{\lambda} T_{d(\mathfrak{v})} T_{w}\right\rangle m_{\lambda} \equiv m_{\lambda} T_{d(\mathfrak{u})} T_{v} T_{w}^{*} T_{d(\mathfrak{v})}^{*} m_{\lambda} \quad \bmod \check{B}_{n}^{\lambda} .
$$

We return to the question of using the bilinear form (3.6) to extract explicit information about the structure of the B-W-W algebras in Sect. 8, but record the following example for later reference.

Example 3.3 Let $n=3$ and $\lambda=(1)$ so that $\check{B}_{n}^{\lambda}=(0)$ and $m_{\lambda}=E_{1}$. We order the basis (3.5) for the module $S^{\lambda}$ as $\mathbf{v}_{1}=E_{1}, \mathbf{v}_{2}=E_{1} T_{2}$ and $\mathbf{v}_{3}=E_{1} T_{2} T_{1}$ and, with respect to this ordered basis, the Gram matrix $\left\langle\mathbf{v}_{i}, \mathbf{v}_{j}\right\rangle$ of the bilinear form (3.6) is

$$
\left[\begin{array}{lcc}
z & r & 1 \\
r z+\left(q-q^{-1}\right)\left(r-r^{-1}\right) & r^{-1} \\
1 & r^{-1} & z
\end{array}\right]
$$

The determinant of the Gram matrix given above is

$$
\frac{(r-1)^{2}(r+1)^{2}\left(q^{3}+r\right)\left(q^{3} r-1\right)}{r^{3}(q-1)^{3}(q+1)^{3}} .
$$

Remark 3.3 (i) Let $\kappa$ be a field and $\hat{r}, \hat{q},\left(\hat{q}-\hat{q}^{-1}\right)$ be units in $\kappa$. The assignments $\varphi: r \mapsto \hat{r}$ and $\varphi: q \mapsto \hat{q}$ determine a homomorphism $R \rightarrow \kappa$, giving $\kappa$ an $R$-module structure. We refer to the specialisation $B_{n}(\hat{q}, \hat{r})=B_{n}(q, r) \otimes_{R} \kappa$ as a B-M-W algebra over $\kappa$. If $0 \leq f \leq[n / 2]$ and $\lambda$ is a partition of $n-2 f$ then the cell module 
$S^{\lambda} \otimes_{R} \kappa$ for $B_{n}(\hat{q}, \hat{r})$ admits a symmetric associative bilinear form which is related to the generic form (3.6) in an obvious way.

(ii) Whenever the context is clear and no possible confusion will arise, the abbreviation $S^{\lambda}$ will be used for the $B_{n}(\hat{q}, \hat{r})$-module $S^{\lambda} \otimes_{R} \kappa$.

The proof of Theorem 3.1 given in [4] rests upon the following facts, respectively Proposition 3.2 of [12] and Proposition 3.3 of [4], stated below for later reference.

Lemma 3.2 Let $f$ be an integer, $0 \leq f \leq[n / 2]$, write $C_{f}$ for the subalgebra of $B_{n}(q, r)$ generated by the elements $T_{2 f+1}, \ldots, T_{n-1}$, and $I_{f}$ for the two sided ideal of $C_{f}$ generated by the element $E_{2 f+1}$. Then the map defined on algebra generators of $\mathscr{H}_{n-2 f}\left(q^{2}\right)$ by

$$
\phi: X_{i} \mapsto T_{2 f+i}+I_{f}, \quad \text { for } 1 \leq i<n-2 f,
$$

and extended to all of $\mathscr{H}_{n-2 f}$ by $\phi\left(h_{1} h_{2}\right)=\phi\left(h_{1}\right) \phi\left(h_{2}\right)$ whenever $h_{1}, h_{2} \in \mathscr{H}_{n-2 f}$, is an algebra isomorphism $\mathscr{H}_{n-2 f}\left(q^{2}\right) \cong C_{f} / I_{f}$.

Lemma 3.3 Let $f$ be an integer, $0 \leq f<[n / 2]$, and $C_{f}$ and $I_{f}$ be as in Lemma 3.2 above. If $i$ is an integer, $2 f<i<n$, and $b \in C_{f}$, then

$$
E_{1} E_{3} \cdots E_{2 f-1} b E_{i} \equiv E_{1} E_{3} \cdots E_{2 f-1} E_{i} b \equiv 0 \quad \bmod B_{n}^{f+1}
$$

Since $\mathscr{H}_{n-2 f}\left(q^{2}\right) \subseteq \mathscr{H}_{n}\left(q^{2}\right)$ is generated by $\left\{X_{j}: 1 \leq j<n-2 f\right\}$, from Lemmas 3.2 and 3.3 we obtain Corollary 3.4 ; cf. Sect. 3 of [4].

Corollary 3.4 If $f$ is an integer, $0 \leq f<[n / 2]$, then there is a well defined $R$ module homomorphism $\vartheta_{f}: \mathscr{H}_{n-2 f}\left(q^{2}\right) \rightarrow B_{n}^{f} / B_{n}^{f+1}$, determined by

$$
\vartheta_{f}: X_{\hat{v}} \rightarrow E_{1} E_{3} \cdots E_{2 f-1} T_{v}+B_{n}^{f+1},
$$

where $v=s_{i_{1}} s_{i_{2}} \cdots s_{i_{d}}$ is a permutation in $\left\langle s_{i}: 2 f<i<n\right\rangle$ and $\hat{w}$ is the permutation $\hat{v}=s_{i_{1}-2 f} s_{i_{2}-2 f} \cdots s_{i_{d}-2 f}$. Additionally, the map $\vartheta_{f}$ satisfies the property

$$
\vartheta_{f}\left(X_{\hat{v}} X_{j}\right)=\vartheta_{f}\left(X_{\hat{v}}\right) T_{2 f+j}
$$

whenever $1 \leq j<n-2 f$.

Remark 3.4 The fact that $\vartheta_{f}$ is an isomorphism of $R$-modules was not used in the proof of Theorem 3.1; however it may be deduced from Theorem 3.1 which implies that the dimension over $R$ of the image space of $\vartheta_{f}$ is equal to the dimension of $\mathscr{H}_{n-2 f}\left(q^{2}\right)$ over $R$.

Lemma 3.5 Let $f$ be an integer, $0<f \leq[n / 2]$. If $b \in B_{n}(q, r), w \in \mathscr{D}_{f, n}$, and $1 \leq i<n$, then there exist $a_{u, v}$ in $R$, for $u$ in $\left\langle s_{i}: 2 f<i<n\right\rangle$ and $v$ in $\mathscr{D}_{f, n}$, uniquely determined by

$$
E_{1} E_{3} \cdots E_{2 f-1} T_{w} b \equiv \sum_{u, v} a_{u, v} E_{1} E_{3} \cdots E_{2 f-1} T_{u} T_{v} \quad \bmod B_{n}^{f+1}
$$


Proof For the uniqueness of the expression (3.9), observe that there is a one-to-one map

$$
E_{1} E_{3} \cdots E_{2 f-1} T_{u} T_{v}+B_{n}^{f+1} \mapsto \sum_{\substack{\mathfrak{s}, \mathfrak{t} \in \operatorname{Std}_{n}(\lambda) \\ \lambda \vdash n-2 f}} a_{\mathfrak{s}, \mathfrak{t}} T_{d(\mathfrak{s})}^{*} m_{\lambda} T_{d(\mathfrak{t})} T_{v}+B_{n}^{f+1},
$$

for $u \in\left\langle s_{j}: 2 f<j<n\right\rangle$ and $v \in \mathcal{D}_{f, n}$, determined by the map $\vartheta_{f}$ and the transition between the basis $\left\{X_{w}: w \in \mathfrak{S}_{n-2 f}\right\}$ and the Murphy basis for $\mathscr{H}_{n-2 f}\left(q^{2}\right)$, where the expression on the right hand side above is an $R$-linear sum of the basis elements for $B_{n}^{f} / B_{n}^{f+1}$ given by Theorem 3.1.

The proof of the lemma makes repeated use of the following fact. If $u^{\prime} \in\left\langle s_{i}: 2 f<\right.$ $i<n\rangle$ and $v^{\prime} \in \mathfrak{S}_{n}$, then $E_{1} E_{3} \cdots E_{2 f-1} T_{u^{\prime}} T_{v^{\prime}}$ is expressible as a sum of the form that appears on the right hand side of (3.9). To see this, first note that, given an integer $i$ with $2 f<i<n$ and $(i+1) v^{\prime}<(i) v^{\prime}$,

$$
T_{u^{\prime}} T_{v^{\prime}}= \begin{cases}T_{u^{\prime} s_{i}} T_{s_{i} v^{\prime}}, & \text { if } \ell\left(u^{\prime}\right)<\ell\left(u^{\prime} s_{i}\right) \\ \left(T_{u^{\prime} s_{i}}+\left(q-q^{-1}\right)\left(T_{u^{\prime}}-r^{-1} T_{u^{\prime} s_{i}} E_{i}\right)\right) T_{s_{i} v}, & \text { otherwise. }\end{cases}
$$

Thus, using Lemma 3.3, we have $a_{u, v} \in R$, for $u \in\left\langle s_{i}: 2 f<i<n\right\rangle$ and $v \in \mathfrak{S}_{n}$, such that

$$
E_{1} E_{3} \cdots E_{2 f-1} T_{u^{\prime}} T_{v^{\prime}} \equiv \sum_{u, v} a_{u, v} E_{1} E_{3} \cdots E_{2 f-1} T_{u} T_{v} \quad \bmod B_{n}^{f+1},
$$

where $(i) v<(i+1) v$, for $2 f<i<n$, whenever $a_{u, v} \neq 0$ in the above expression. Noting that $E_{1} E_{3} \cdots E_{2 f-1} T_{v}=r^{-1} E_{1} E_{3} \cdots E_{2 f-1} T_{s_{2 i-1} v}$ if $1 \leq i \leq f$ and $\ell\left(s_{2 i-1} v\right)<\ell(v)$, and applying Proposition 3.7 or Corollary 3.1 of [4], we may assume that $v \in \mathscr{D}_{f, n}$, whenever $a_{u, v} \neq 0$ in the above expression.

Proceeding with the proof of the lemma, first consider the case where $b=E_{i}$ for some $1 \leq i<n$. Let $k=(i) w^{-1}$ and $l=(i+1) w^{-1}$. If $(i+1) w^{-1}<(i) w^{-1}$, then $T_{w} E_{i}=r^{-1} T_{w s_{i}} E_{i}$, where $w s_{i} \in \mathcal{D}_{f, n}$. We may therefore suppose that $k<l$. Using Proposition 3.4 of [4],

$$
T_{w} E_{i}= \begin{cases}E_{k} T_{w}, & \text { if } l=k+1 \\ T_{l-1}^{\varepsilon_{l-1}} T_{l-2}^{\varepsilon_{l-2}} \cdots T_{k+1}^{\varepsilon_{k+1}} E_{k} T_{w^{\prime}}, & \text { otherwise }\end{cases}
$$

where $w^{\prime}=s_{k+1} s_{k+2} \cdots s_{l-1} w$ and, for $k<j<l$,

$$
\varepsilon_{j}= \begin{cases}1, & \text { if } i+1<(j) w \\ -1, & \text { otherwise }\end{cases}
$$

Considering the two cases in (3.10) separately, multiply both sides of the expression (3.10) by $E_{1} E_{3} \cdots E_{2 f-1}$. If $l=k+1$, then

$$
E_{1} E_{3} \cdots E_{2 f-1} T_{w} E_{i}= \begin{cases}z E_{1} E_{3} \cdots E_{2 f-1} T_{w}, & \text { if } k<2 f \text { and } k \text { is odd; } \\ E_{1} E_{3} \cdots E_{2 f-1} T_{k} T_{k-1} T_{w}, & \text { if } k \leq 2 f \text { and } k \text { is even; } \\ E_{1} E_{3} \cdots E_{2 f-1} E_{k} T_{w}, & \text { if } 2 f<k\end{cases}
$$


By Proposition 3.8 of [4], there exist $a_{v^{\prime}} \in R$, for $v^{\prime} \in \mathfrak{S}_{n}$ such that, given $w^{\prime} \in \mathfrak{S}_{n}$ satisfying $(2 j) w^{\prime}+1=(2 j+1) w^{\prime}$, together with $\varepsilon_{2 j-1}, \varepsilon_{2 j} \in\{ \pm 1\}$,

$$
E_{2 j-1} T_{2 j}^{\varepsilon_{2 j}} T_{2 j-1}^{\varepsilon_{2 j-1}} T_{w^{\prime}}=\sum_{v^{\prime} \in \mathfrak{S}_{n}} a_{v^{\prime}} E_{2 j-1} T_{v^{\prime}}
$$

Using (3.12) with $k=2 j$, the term appearing in the second case on the right hand side of (3.11) can be rewritten as

$$
E_{1} E_{3} \cdots E_{2 f-1} T_{k} T_{k-1} T_{w^{\prime}}=\sum_{v^{\prime} \in \mathfrak{S}_{n}} a_{v^{\prime}} E_{1} E_{3} \cdots E_{2 j-1} T_{v^{\prime}}
$$

As already noted, the right hand side of the above expression may be rewritten modulo $B_{n}^{f+1}$ as an $R$-linear combination of the required form. On the other hand, the term appearing on the right in the last case in (3.11) above is zero modulo $B_{n}^{f+1}$.

The second case on the right hand side of (3.10) gives rise to three sub-cases as follows. First, if $2 f<k<n$, then

$$
E_{1} E_{3} \cdots E_{2 f-1} T_{l-1}^{\varepsilon_{l-1}} T_{l-2}^{\varepsilon_{l-2}} \cdots T_{k+1}^{\varepsilon_{k+1}} E_{k} T_{w^{\prime}} \equiv 0 \quad \bmod B_{n}^{f+1} ;
$$

if $1 \leq k<2 f$ and $k$ is odd, then

$$
E_{k} T_{l-1}^{\varepsilon_{l-1}} T_{l-2}^{\varepsilon_{l-2}} \cdots T_{k+1}^{\varepsilon_{k+1}} E_{k} T_{w^{\prime}}=r^{\varepsilon_{k+1}} E_{k} T_{l-1}^{\varepsilon_{l-1}} T_{l-2}^{\varepsilon_{l-2}} \cdots T_{k+2}^{\varepsilon_{k+2}} T_{w^{\prime}} ;
$$

if $1<k \leq 2 f$ and $k$ is even, then

$$
E_{k-1} T_{l-1}^{\varepsilon_{l-1}} T_{l-2}^{\varepsilon_{l-2}} \cdots T_{k+1}^{\varepsilon_{k+1}} E_{k} T_{w^{\prime}}=E_{k-1} T_{l-1}^{\varepsilon_{l-1}} T_{l-2}^{\varepsilon_{l-2}} \cdots T_{k+1}^{\varepsilon_{k+1}} T_{k} T_{k-1} T_{w^{\prime}} .
$$

When $1 \leq k<2 f$ and $k$ is odd, using (3.10) and (3.13), and successively applying (3.12) with $j=k, k-2, \ldots$, we obtain

$$
\begin{aligned}
& E_{1} E_{3} \cdots E_{2 f-1} T_{l-1}^{\varepsilon_{l-1}} T_{l-2}^{\varepsilon_{l-2}} \cdots T_{k+1}^{\varepsilon_{k+1}} E_{k} T_{w^{\prime}} \\
& \quad=\sum_{v^{\prime} \in \mathfrak{S}_{n}} a_{v^{\prime}} T_{l-1}^{\varepsilon_{l-1}} T_{l-2}^{\varepsilon_{l-2}} \cdots T_{2 f+1}^{\varepsilon_{2 f+1}} E_{1} E_{3} \cdots E_{2 f-1} T_{v^{\prime}}
\end{aligned}
$$

where $T_{l-1}^{\varepsilon_{l-1}} T_{l-2}^{\varepsilon_{l-2}} \cdots T_{2 f+1}^{\varepsilon_{2 f+1}}$ can be expressed as a sum

$$
T_{l-1}^{\varepsilon_{l-1}} T_{l-2}^{\varepsilon_{l-2}} \cdots T_{2 f+1}^{\varepsilon_{2 f+1}}=\sum_{u^{\prime} \in\left\langle s_{j}: 2 f<j<n\right\rangle} a_{u^{\prime}} T_{u^{\prime}}+b^{\prime},
$$

and $b^{\prime}$ lies in the two sided ideal of $\left\langle T_{j}: 2 f<j<n\right\rangle$ generated by $E_{2 f+1}$. Since $b^{\prime}$ satisfies $E_{1} E_{3} \cdots E_{2 f-1} b^{\prime} \in B_{n}^{f+1}$, it follows that

$$
\begin{aligned}
& E_{1} E_{3} \cdots E_{2 f-1} T_{l-1}^{\varepsilon_{l-1}} T_{l-2}^{\varepsilon_{l-2}} \cdots T_{k+1}^{\varepsilon_{k+1}} E_{k} T_{w^{\prime}} \\
& \equiv \sum_{\substack{v^{\prime} \in \mathfrak{S}_{n} \\
u^{\prime} \in\left\langle s_{j}: 2 f<j<n\right\rangle}} a_{u^{\prime}, v^{\prime}} E_{1} E_{3} \cdots E_{2 f-1} T_{u^{\prime}} T_{v^{\prime}} \bmod B_{n}^{f+1} .
\end{aligned}
$$


As already noted, the right hand side of the above expression may be rewritten modulo $B_{n}^{f+1}$ as an $R$-linear combination of the required form. In the same way, if $1<k \leq 2 f$ and $k$ is even, then using (3.14), we obtain the product

$$
E_{1} E_{3} \cdots E_{2 f-1} T_{l-1}^{\varepsilon_{l-1}} T_{l-2}^{\varepsilon_{l-2}} \cdots T_{k+1}^{\varepsilon_{k+1}} T_{k} T_{k-1} T_{w^{\prime}}
$$

which is also expressible as a sum of the required form using the arguments above. Thus we have shown that the lemma holds in case $1 \leq i<n$ and $b=E_{i}$.

Let $w \in \mathscr{D}_{f, n}$. If $1 \leq i<n$, and $\ell(w)<\ell\left(w s_{i}\right)$ then

$$
E_{1} E_{3} \cdots E_{2 f-1} T_{w} T_{i}=E_{1} E_{3} \cdots E_{2 f-1} T_{w s_{i}},
$$

and, if $\ell\left(w s_{i}\right)<\ell(w)$, then

$$
E_{1} E_{3} \cdots E_{2 f-1} T_{w} T_{i}=E_{1} E_{3} \cdots E_{2 f-1}\left(T_{w s_{i}}+\left(q-q^{-1}\right)\left(T_{w}-r^{-1} T_{w s_{i}} E_{i}\right)\right) .
$$

We have already observed that the terms appearing on the right hand side in each of the two above expressions may be expressed as an $R$-linear combination of the required form. Thus we have shown that the lemma holds when $b \in\left\{T_{i}: 1 \leq i<n\right\}$.

Now, given that the lemma holds when $b \in\left\{T_{i}: 1 \leq i<n\right\}$, Lemma 3.3 shows that any product

$$
E_{1} E_{3} \cdots E_{2 f-1} T_{u} T_{v} T_{i}, \quad \text { for } u \in\left\langle s_{i}: 2 f<i<n\right\rangle \text { and } v \in \mathscr{D}_{f, n},
$$

can also be written as an $R$-linear combination of the form appearing on the right hand side of (3.9). Since $\left\{T_{i}: 1 \leq i<n\right\}$ generates $B_{n}(q, r)$, the proof of the lemma is complete.

If $f$ is an integer, $0 \leq f \leq[n / 2]$, and $\mu$ is a partition of $n-2 f$, define $L^{\mu}$ to be the right $B_{n}(q, r)$-submodule of $B_{n}^{f} / B_{n}^{f+1}$ generated by the element $m_{\mu}+B_{n}^{f+1}$. The next result will be used in Sect. 5 below; the element $m_{\text {St }}$ defined in the next lemma is an analogue to the element $c_{\mathrm{St}} \in \mathcal{H}_{n}\left(q^{2}\right)$ given in (2.6).

Lemma 3.6 Let $f$ be an integer, $0 \leq f \leq[n / 2]$, and given partitions $\lambda, \mu$ of $n-2 f$, with $\lambda \unrhd \mu$, define

$$
m_{\mathrm{St}}=\sum_{\substack{\mathfrak{s} \in \operatorname{Std}_{n}(\lambda) \\ \mu(\hat{\mathfrak{s}})=\mathrm{S}}} q^{\ell(d(\mathfrak{s}))} T_{d(\mathfrak{s})}^{*} m_{\lambda} T_{d(\mathfrak{t})}, \quad \text { for } \mathrm{S} \in \mathcal{T}_{0}(\lambda, \mu) \text { and } \mathfrak{t} \in \operatorname{Std}_{n}(\lambda) .
$$

Then the collection

$$
\left\{\begin{array}{l|l}
m_{\mathrm{St}} T_{v}+B_{n}^{f+1} & \begin{array}{c}
\text { for } \mathrm{S} \in \mathcal{T}_{0}(\lambda, \mu), \mathfrak{t} \in \operatorname{Std}_{n}(\lambda), \\
\lambda \vdash n-2 f \text { and } v \in \mathscr{D}_{f, n}
\end{array}
\end{array}\right\}
$$

freely generates $L^{\mu}$ as an $R-$ module.

Proof If $b \in B_{n}(q, r)$ and $w \in \mathscr{D}_{f, n}$, then by the previous lemma, there exist $a_{u, v} \in$ $R$, for $u \in\left\langle s_{i}: 2 f<i<n\right\rangle$ and $v \in \mathscr{D}_{f, n}$ such that

$$
E_{1} E_{3} \cdots E_{2 f-1} T_{w} b \equiv \sum_{u, v} a_{u, v} E_{1} E_{3} \cdots E_{2 f-1} T_{u} T_{v} \quad \bmod B_{n}^{f+1} .
$$


Multiplying both sides of the above expression by $x_{\mu}$ on the left, and using the property (3.8) and Theorem 2.2, we obtain $a_{\mathrm{S}, \mathrm{t}} \in R$, for $\mathrm{S} \in \mathcal{T}_{0}(\lambda, \mu), \mathfrak{t} \in \operatorname{Std}_{n}(\lambda)$ and $\lambda \vdash n-2 f$, such that

$$
\begin{aligned}
m_{\mu} T_{w} b+B_{n}^{f+1} & =\sum_{u, v} a_{u, v} E_{1} E_{3} \cdots E_{2 f-1} x_{\mu} T_{u} T_{v}+B_{n}^{f+1} \\
& =\sum_{u, v} a_{u, v} \vartheta_{f}\left(c_{\mu} X_{\hat{u}}\right) T_{v}=\sum_{u, v} a_{u, v} \sum_{\substack{\mathrm{S} \in \mathcal{T}_{0}(\lambda, \mu) \\
\mathfrak{t} \in \operatorname{Std}_{n}(\lambda)}} a_{\mathrm{S}, \mathfrak{t}} \vartheta_{f}\left(c_{\mathrm{S} \hat{\mathfrak{t}}}\right) T_{v} \\
& =\sum_{u, v} a_{u, v} \sum_{\substack{\mathrm{S} \in \mathcal{T}_{0}(\lambda, \mu) \\
\mathfrak{t} \in \operatorname{Std}_{n}(\lambda)}} a_{\mathrm{S}, \mathfrak{t}} m_{\mathrm{St}} T_{v}+B_{n}^{f+1} .
\end{aligned}
$$

This proves the spanning property of the set (3.15). The fact that each element of the set (3.15) lies in $L^{\mu}$ follows from an argument similar to the above, using Theorem 2.2 and the property (3.8). We now outline the proof of the linear independence of the elements of (3.15) over $R$.

(i) Let $\left\{\mathrm{S}_{i}: 1 \leq i \leq k\right\}$ be the semistandard tableaux of type $\mu$, ordered so that $\mathrm{S}_{i} \in \mathcal{T}_{0}\left(\lambda_{i}, \mu\right)$ and $j \geq i$ whenever $\lambda_{i} \unrhd \lambda_{j}$, and take $L_{i}$ to denote the $R$-module generated by

$$
\left\{m_{\mathrm{S}_{j}} T_{v}+B_{n}^{f+1}: 1 \leq j \leq i, \mathfrak{t} \in \operatorname{Std}_{n}\left(\lambda_{j}\right) \text { and } v \in \mathscr{D}_{f, n}\right\}
$$

(ii) Using the property (3.8) and Theorem 2.2 as above, it is shown that the $R-$ module homomorphism $L_{i} / L_{i-1} \rightarrow S^{\lambda_{i}}$ defined, for $\mathfrak{t} \in \operatorname{Std}_{n}\left(\lambda_{i}\right)$ and $w \in \mathscr{D}_{f, n}$, by

$$
m_{\mathrm{S}_{i} \mathfrak{t}} T_{w}+L_{i-1} \mapsto m_{\lambda_{i}} T_{d(\mathfrak{t})} T_{w}+\check{B}_{n}^{\lambda_{i}}
$$

is an isomorphism of right $B_{n}(q, r)$-modules. Thus, analogous to the filtration of each permutation module of the Iwahori-Hecke algebra of the symmetric group given in Corollary 4.10 of [8], there is a filtration of $L^{\mu}$ by $B_{n}(q, r)$-modules

$$
(0)=L_{0} \subseteq L_{1} \subseteq \cdots \subseteq L_{k}=L^{\mu}
$$

wherein each factor $L_{i} / L_{i-1}$ is isomorphic to a cell module $S^{\lambda_{i}}$ for $B_{n}(q, r)$.

(iii) From (3.17), it is deduced that $\operatorname{dim}_{R}\left(L^{\mu}\right)=\sum_{i=1}^{k} \operatorname{dim}_{R}\left(S^{\lambda_{i}}\right)$. Since this sum coincides with the order of the set (3.15) obtained by simply counting, the linear independence over $R$ of the elements of (3.15) now follows.

\section{Representation theory over a field}

We state for later reference some consequences, for B-M-W algebras over a field, of the theory of cellular algebras constructed in [5]. These results of C.C. Xi appeared in [13]. 
Proposition 4.1 Let $B_{n}(\hat{q}, \hat{r})$ be a $B-M-W$ algebra over a field $\kappa$. If $f$ is an integer, $0 \leq f \leq[n / 2]$, and $\lambda$ is a partition of $n-2 f$, then the radical

$$
\operatorname{rad}\left(S^{\lambda}\right)=\left\{\mathbf{v} \in S^{\lambda}:\langle\mathbf{v}, \mathbf{u}\rangle=0 \text { for all } \mathbf{u} \in S^{\lambda}\right\}
$$

of the form on $S^{\lambda}$ determined by (3.6) is a $B_{n}(\hat{q}, \hat{r})$-submodule of $S^{\lambda}$.

Proposition 4.2 Let $B_{n}(\hat{q}, \hat{r})$ be a $B-M-W$ algebra over a field $\kappa$, and suppose that $f, f^{\prime}$ are integers $0 \leq f, f^{\prime} \leq[n / 2]$, and $\lambda, \mu$ are partitions of $n-2 f$ and $n-2 f^{\prime}$ respectively. If $M$ is a $B_{n}(\hat{q}, \hat{r})-$ submodule of $S^{\lambda}$, and $\psi: S^{\mu} \rightarrow S^{\lambda} / M$ is a nontrivial $B_{n}(\hat{q}, \hat{r})$-module homomorphism, then $\lambda \unrhd \mu$.

Let $B_{n}(\hat{q}, \hat{r})$ be a B-M-W algebra over a field $\kappa$. If $f$ is an integer with $0 \leq f \leq$ [n/2], and $\lambda$ is a partition of $n-2 f$, define the $B_{n}(\hat{q}, \hat{r})$-module $D^{\lambda}=S^{\lambda} / \operatorname{rad}\left(S^{\lambda}\right)$.

Theorem 4.3 If $\kappa$ is a field and $B_{n}(\hat{q}, \hat{r})$ is a $B-M-W$ algebra over $\kappa$, then $\left\{D^{\lambda}\right.$ : $D^{\lambda} \neq 0, \lambda \vdash n-2 f$ and $\left.0 \leq f \leq[n / 2]\right\}$ is a complete set of pairwise inequivalent irreducible $B_{n}(\hat{q}, \hat{r})$-modules.

Theorem 4.4 Let $\kappa$ be a field and $B_{n}(\hat{q}, \hat{r})$ be a $B-M-W$ algebra over $\kappa$. Then the following statements are equivalent.

1. $B_{n}(\hat{q}, \hat{r})$ is (split) semisimple.

2. $S^{\lambda}=D^{\lambda}$ for all $\lambda \vdash n-2 f$ and $0 \leq f \leq[n / 2]$.

3. $\operatorname{rad}\left(S^{\lambda}\right)=0$ for all $\lambda \vdash n-2 f$ and $0 \leq f \leq[n / 2]$.

\section{Restriction}

Given an integer, $1 \leq i \leq n$, regard $B_{i}(q, r)$ as the subalgebra of $B_{n}(q, r)$ generated by the elements $T_{1}, T_{2}, \cdots, T_{i-1}$, thereby obtaining the tower

$$
R=B_{1}(q, r) \subseteq B_{2}(q, r) \subseteq \cdots \subseteq B_{n}(q, r) .
$$

If $V$ is a $B_{n}(q, r)$-module, using the identification (5.1), we write $\operatorname{Res}(V)$ for the restriction of $V$ to $B_{n-1}(q, r)$.

In order to construct a basis for the cell module $S^{\lambda}$ which behaves well with respect to both restriction in the tower (5.1) and with respect to the action of the JucysMurphy operators in $B_{n}(q, r)$, we first consider in this section the behaviour of the cell module $S^{\lambda}$ under restriction from $B_{n}(q, r)$ to $B_{n-1}(q, r)$. This description of the restriction functor on the cell modules for the $\mathrm{B}-\mathrm{M}-\mathrm{W}$ algebras given here will be used in Sect. 6 to construct a basis for the cell module $S^{\lambda}$ which behaves regularly with respect to restriction in the tower (5.1) and with respect to the Jucys-Murphy operators in $B_{n}(q, r)$.

The material of this section is motivated by the Wedderburn decomposition of the semisimple B-M-W algebras over a field $\mathbb{C}(\hat{q}, \hat{r})$ given by $\mathrm{H}$. Wenzl in [12], and by the bases for the B-M-W algebras indexed by paths in the Bratteli diagram associated with the B-M-W algebras, constructed in the semisimple setting over $\mathbb{C}(\hat{q}, \hat{r})$, by 
R. Leduc and A. Ram in [6]. As made clear by [6] and [12], paths in the Bratteli diagram associated with the $\mathrm{B}-\mathrm{M}-\mathrm{W}$ algebras provide the most natural generalisation to our setting of the standard tableaux from the representation theory of the symmetric group. However, while the bases constructed in Sect. 6 and in [6] are both indexed by paths in the appropriate Bratteli diagram, we have sought a generic basis over a ring $R=\mathbb{Z}\left[q^{ \pm 1}, r^{ \pm 1},\left(q-q^{-1}\right)^{-1}\right]$. Thus the construction here will not require the assumptions about semisimplicity which are necessary in [6].

Let $f$ be an integer, $0 \leq f \leq[n / 2]$, and $\lambda$ be a partition of $n-2 f$. Henceforth, write $\mu \rightarrow \lambda$ to mean that either

1. $\mu$ is a partition of $n-2 f+1$ and the diagram $[\mu]$ is obtained by adding a node to the diagram $[\lambda]$ or,

2. $\mu$ is a partition of $n-2 f-1$ and the diagram $[\mu]$ is obtained by deleting a node from the diagram $[\lambda]$,

as illustrated in the truncated Bratteli diagram associated with $B_{n}(q, r)$ displayed in (5.2) below (Sect. 5 of [6]).

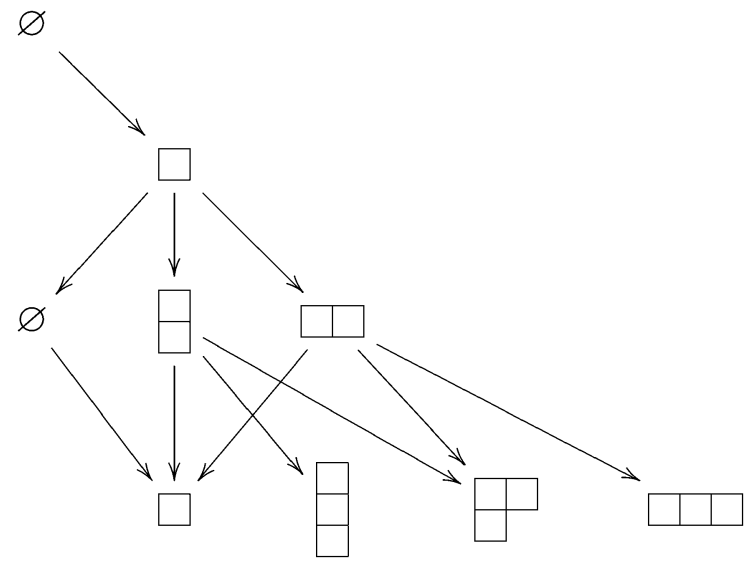

Let $f$ be an integer, $0 \leq f \leq[n / 2]$, and $\lambda$ be a partition of $n-2 f$ with $t$ removable nodes and suppose that

$$
\mu^{(1)} \triangleright \mu^{(2)} \triangleright \cdots \triangleright \mu^{(t)}
$$

is the ordering of the set $\{\mu: \mu \rightarrow \lambda$ and $|\lambda|>|\mu|\}$ by dominance order on partitions. For each partition $\mu^{(k)}$ in the list (5.3), define an element

$$
y_{\mu^{(k)}}^{\lambda}=m_{\lambda} T_{d(\mathfrak{s})}+\check{B}_{n}^{\lambda} \quad \quad \text { where }\left.\mathfrak{s}\right|_{n-1}=\mathfrak{t}^{\mu^{(k)}},
$$

and let $N^{\mu^{(k)}}$ denote the $B_{n-1}(q, r)$-submodule of $S^{\lambda}$ generated by

$$
\left\{y_{\mu^{(k)}}^{\lambda} T_{d(\mathfrak{u})}: \mathfrak{u} \in \operatorname{Std}_{n-1}\left(\mu^{(k)}\right)\right\}
$$

write $\check{N}^{\mu^{(k)}}$ for the $B_{n-1}(q, r)$-submodule of $S^{\lambda}$ generated by

$$
\left\{y_{\mu^{(j)}}^{\lambda} T_{d(\mathfrak{u})}: \mathfrak{u} \in \operatorname{Std}_{n-1}\left(\mu^{(j)}\right) \text { and } j<k\right\} .
$$


Example 5.1 Let $n=10, f=2$ and $\lambda=(3,2,1)$. Then

$$
m_{\lambda}=E_{1} E_{3} \sum_{w \in \mathfrak{S}_{\lambda}} q^{\ell(w)} T_{w}=E_{1} E_{3}\left(1+q T_{5}\right)\left(1+q T_{6}+q^{2} T_{6} T_{5}\right)\left(1+q T_{8}\right)
$$

and the elements $y_{\mu^{(k)}}^{\lambda}$, for each partition $\mu^{(k)} \rightarrow \lambda$ with $|\lambda|>\left|\mu^{(k)}\right|$, are as follows.

1. If $\mu^{(1)}=(3,2)$, then $\mathfrak{t}^{\mu}=\left.\mathfrak{s}\right|_{n-1}$, where $\mathfrak{s}=$\begin{tabular}{|l|l|l|}
\hline 5 & 6 & 7 \\
\hline 8 & 9 & , so \\
\cline { 1 - 1 } 10 &
\end{tabular}

$$
y_{\mu^{(1)}}^{\lambda}=m_{\lambda}+\check{B}_{n}^{\lambda} \text {. }
$$

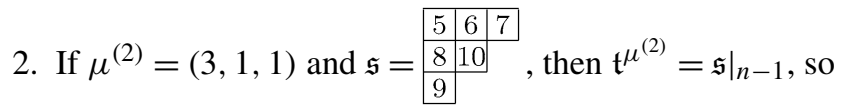

$$
y_{\mu^{(2)}}^{\lambda}=m_{\lambda} T_{d(\mathfrak{s})}+\check{B}_{n}^{\lambda}=m_{\lambda} T_{9}+\check{B}_{n}^{\lambda}
$$

3. If $\mu^{(3)}=(2,2,1)$, then $\mathfrak{t}^{\mu^{(3)}}=\left.\mathfrak{s}\right|_{n-1}$, where $\mathfrak{s}=$\begin{tabular}{|l|l|l|}
\hline 5 & 6 & 10 \\
\hline 7 & 8 \\
\hline 9 & , so
\end{tabular}

$$
y_{\mu^{(3)}}^{\lambda}=m_{\lambda} T_{d(\mathfrak{s})}+\check{B}_{n}^{\lambda}=m_{\lambda} T_{7} T_{8} T_{9}+\check{B}_{n}^{\lambda} .
$$

Write $\mathscr{D}_{f, n-1}=\left\{v \in \mathscr{D}_{f, n}:(n) v=n\right\}$, so identifying $\mathscr{D}_{f, n-1} \subseteq \mathscr{D}_{f, n}$.

Lemma 5.1 Let $f$ be an integer, $0 \leq f \leq[n / 2]$, and $\lambda$ be a partition of $n-2 f$. If $\mu$ is a partition with $|\lambda|>|\mu|$ and $\mu \rightarrow \lambda$, then $N^{\mu} / \check{N}^{\mu}$ is the $R$-module freely generated by

$$
\left\{y_{\mu}^{\lambda} T_{d(\mathfrak{u})} T_{w}+\check{N}^{\mu}: \mathfrak{u} \in \operatorname{Std}_{n-1}(\mu) \text { and } w \in \mathscr{D}_{f, n-1}\right\} .
$$

Additionally, the map defined, for $\mathfrak{u} \in \operatorname{Std}_{n-1}(\mu)$ and $w \in \mathscr{D}_{f, n-1}$, by

$$
y_{\mu}^{\lambda} T_{d(\mathfrak{u})} T_{w}+\check{N}^{\mu} \mapsto m_{\mu} T_{d(\mathfrak{u})} T_{w}+\check{B}_{n-1}^{\mu}
$$

determines an isomorphism $N^{\mu} / \check{N}^{\mu} \cong S^{\mu}$ of $B_{n-1}(q, r)$-modules.

Proof Let $b \in B_{n-1}(q, r)$ and $w \in \mathscr{D}_{f, n-1}$. By Lemma 3.5, there exist $a_{u, v} \in R$, for $u \in\left\langle s_{i}: 2 f<i<n-1\right\rangle$ and $v \in \mathscr{D}_{f, n-1}$, determined uniquely by

$$
E_{1} E_{3} \cdots E_{2 f-1} T_{w} b \equiv \sum_{u, v} a_{u, v} E_{1} E_{3} \cdots E_{2 f-1} T_{u} T_{v} \quad \bmod B_{n-1}^{f+1} .
$$

Let $\mathfrak{v} \in \operatorname{Std}_{n}(\lambda)$ satisfy $\left.\mathfrak{v}\right|_{n-1}=\mathfrak{t}^{\mu}$ so that $y_{\mu}^{\lambda}=m_{\lambda} T_{d(\mathfrak{v})}+\check{B}_{n}^{\lambda}$, and let $\mathfrak{u} \in \operatorname{Std}_{n-1}(\mu)$. Since $B_{n-1}^{f+1} \subset B_{n}^{f+1}$, we use (5.6) and Lemma 2.3 to obtain $a_{\mathfrak{s}}, a_{\mathfrak{t}} \in R$, for $\mathfrak{s} \in$ Springer 
$\operatorname{Std}_{n-1}(\mu)$ and $\mathfrak{t} \in \operatorname{Std}_{n}(\lambda)$ such that

$$
\begin{aligned}
m_{\lambda} T_{d(\mathfrak{v})} T_{d(\mathfrak{u})} T_{w} b+B_{n}^{f+1} & \\
= & \sum_{u, v} a_{u, v} \vartheta_{f}\left(c_{\lambda} X_{d(\hat{\mathfrak{v}})} X_{d(\hat{\mathfrak{u}})} X_{\hat{u}}\right) T_{v} \\
= & \sum_{u, v} a_{u, v} \sum_{\mathfrak{s} \in \operatorname{Std}_{n-1}(\mu)} a_{\mathfrak{s}} \vartheta_{f}\left(c_{\lambda} X_{d(\hat{\mathfrak{v}})} X_{d(\hat{\mathfrak{s}})}\right) T_{v} \\
& +\sum_{u, v} a_{u, v} \sum_{\substack{\mathfrak{t} \in \operatorname{Std}_{n}(\lambda) \\
\operatorname{Shape}\left(\mathfrak{t}_{n-1}\right) \triangleright \mu}} a_{\mathfrak{t}} \vartheta_{f}\left(c_{\lambda} X_{d(\hat{\mathfrak{t}})}\right) T_{v}+\sum_{u, v} a_{u, v} \vartheta_{f}(h) T_{v},
\end{aligned}
$$

where $h \in \check{\mathscr{H}}_{n-2 f}^{\lambda}$ and $\vartheta_{f}(h) \subseteq \check{B}_{n}^{\lambda}$. We thus obtain,

$$
\begin{aligned}
m_{\lambda} T_{d(\mathfrak{v})} T_{d(\mathfrak{u})} T_{w} b+B_{n}^{f+1}= & \sum_{u, v} a_{u, v} \sum_{\mathfrak{s} \in \operatorname{Std}_{n-1}(\mu)} a_{\mathfrak{s}} m_{\lambda} T_{d(\mathfrak{v})} T_{d(\mathfrak{s})} T_{v} \\
& +\sum_{u, v} a_{u, v} \sum_{\substack{\mathfrak{t} \in \operatorname{Std}_{n}(\lambda) \\
\operatorname{Shape}\left(\left.\mathfrak{t}\right|_{n-1}\right) \triangleright \mu}} a_{\mathfrak{t}} m_{\lambda} T_{d(\mathfrak{t})} T_{v}+b^{\prime}
\end{aligned}
$$

where $b^{\prime} \in \check{B}_{n}^{\lambda}$. Since $\check{N}^{\mu}$ is generated as a $B_{n-1}(q, r)$ module by $\left\{m_{\lambda} T_{d(\mathfrak{t})}+\check{B}_{n}^{\lambda}\right.$ : $\mathfrak{t} \in \operatorname{Std}_{n}(\lambda)$ and Shape $\left.\left(\left.\mathfrak{t}\right|_{n-1}\right) \triangleright \mu\right\}$, it follows that

$$
y_{\mu}^{\lambda} T_{d(\mathfrak{u})} T_{w} b \equiv \sum_{u, v} a_{u, v} \sum_{\mathfrak{s} \in \operatorname{Std}_{n-1}(\mu)} a_{\mathfrak{s}} y_{\mu}^{\lambda} T_{d(\mathfrak{s})} T_{v} \quad \bmod \check{N}^{\mu} .
$$

Using (5.6) and Lemma 2.3 again the $a_{\mathfrak{s}}$, for $\mathfrak{s} \in \operatorname{Std}_{n-1}(\mu)$, given above also satisfy

$$
\begin{aligned}
m_{\mu} T_{d(\mathfrak{u})} T_{w} b+B_{n-1}^{f+1} & =\sum_{u, v} a_{u, v} \vartheta_{f}\left(c_{\mu} X_{d(\hat{\mathfrak{u}})} X_{\hat{u}}\right) T_{v} \\
& =\sum_{u, v} a_{u, v} \sum_{\mathfrak{s} \in \operatorname{Std}_{n-1}(\mu)} a_{\mathfrak{s}} \vartheta_{f}\left(c_{\mu} X_{d(\hat{\mathfrak{s}})}\right) T_{v}+\sum_{u, v} a_{u, v} \vartheta_{f}\left(h^{\prime}\right) T_{v},
\end{aligned}
$$

where $h^{\prime} \in \check{\mathscr{H}}_{n-2 f-1}^{\mu}$. Since $\vartheta_{f}\left(h^{\prime}\right) \subseteq \check{B}_{n-1}^{\mu}$,

$$
m_{\mu} T_{d(\mathfrak{u})} T_{w} b+\check{B}_{n-1}^{\mu}=\sum_{u, v} a_{u, v} \sum_{\mathfrak{s} \in \operatorname{Std}_{n-1}(\mu)} a_{\mathfrak{s}} m_{\mu} T_{d(\mathfrak{s})} T_{v}+\check{B}_{n-1}^{\mu} .
$$

Comparing coefficients in (5.7) and (5.8) shows that the $R$-module isomorphism (5.5) is also a $B_{n-1}(q, r)$-module homomorphism.

Corollary 5.2 Let $f$ be an integer, $0 \leq f \leq[n / 2]$, and $\lambda$ be a partition of $n-2 f$. If $\mu$ is a partition of $n-2 f-1$ with $\mu \rightarrow \lambda$, then $N^{\mu}$ is the $R$-module freely generated by

$$
\left\{m_{\lambda} T_{d(\mathfrak{s})} T_{v}+\check{B}_{n}^{\lambda}: \mathfrak{s} \in \operatorname{Std}_{n}(\lambda), \operatorname{Shape}\left(\left.\mathfrak{s}\right|_{n-1}\right) \unrhd \mu \text { and } v \in \mathscr{D}_{f, n-1}\right\}
$$


Let $f$ be an integer, $0<f \leq[n / 2]$, with $\lambda$ a partition of $n-2 f$ having $t$ removable nodes and $(p-t)$ addable nodes, and suppose that

$$
\mu^{(t+1)} \triangleright \mu^{(t+2)} \triangleright \cdots \triangleright \mu^{(p)}
$$

is the ordering of $\{\mu: \mu \rightarrow \lambda$ and $|\mu|>|\lambda|\}$ by dominance order on partitions. By the definition of the dominance order on partitions which we use here, the list (5.3) can be extended as

$$
\mu^{(1)} \triangleright \mu^{(2)} \triangleright \cdots \triangleright \mu^{(t)} \triangleright \mu^{(t+1)} \triangleright \mu^{(t+2)} \triangleright \cdots \triangleright \mu^{(p)} .
$$

In the manner of Lemma 5.1, we seek to assign to each partition $\mu^{(k)}$, with $k>t$, in the list (5.9), a $B_{n-1}(q, r)$-submodule $N^{\mu^{(k)}}$ of $S^{\lambda}$, and an associated generator $y_{\mu^{(k)}}^{\lambda}+\check{N}^{\mu^{(k)}}$ in $S^{\lambda} / \check{N}^{\mu^{(k)}}$. To this end, first let

$$
w_{p}=s_{n-2} s_{n-3} \cdots s_{2 f-1} s_{n-1} s_{n-2} \cdots s_{2 f}
$$

and write $N^{\mu^{(p)}}$ for the $B_{n-1}(q, r)$-submodule of $S^{\lambda}$ generated by the element

$$
y_{\mu}^{\lambda(p)}=m_{\lambda} T_{w_{p}}^{-1}+\check{B}_{n}^{\lambda} .
$$

From the defining relations for $B_{n}(q, r)$, or using the presentation for $B_{n}(q, r)$ in terms of tangles given in [1], it is readily observed that $E_{2 f-1} T_{w_{p}}^{-1}=E_{2 f-1} T_{w_{p}^{-1}}$, and consequently that $m_{\lambda} T_{w_{p}}^{-1}=m_{\lambda} T_{w_{p}^{-1}}$. Since $w_{p}^{-1}$ is an element of $\mathscr{D}_{f, n}$ with (2f) $w_{p}^{-1}=n$, Corollary 5.2 implies that the element $m_{\lambda} T_{w_{p}^{-1}}+\check{B}_{n}^{\lambda}$ is contained in the complement of $N^{\mu^{(t)}}$ in $S^{\lambda}$. Furthermore, using the relation $E_{i} T_{i+1} T_{i}=T_{i+1} T_{i} E_{i+1}$ it can be seen that

$$
\begin{aligned}
E_{2 f-1} T_{w_{p}^{-1}} & =E_{2 f-1} T_{2 f} T_{2 f+1} \cdots T_{n-2} T_{n-1} T_{2 f-1} T_{2 f} \cdots T_{n-3} T_{n-2} \\
& =T_{2 f} T_{2 f-1} T_{2 f+1} T_{2 f} \cdots T_{n-2} T_{n-3} E_{n-2} T_{n-1} T_{n-2},
\end{aligned}
$$

whence, if $\mathfrak{s} \in \operatorname{Std}_{n}(\lambda)$,

$$
\begin{aligned}
m_{\lambda} T_{d(\mathfrak{s})} T_{w_{p}}^{-1} & =m_{\lambda} T_{d(\mathfrak{s})} T_{w_{p}^{-1}}=E_{1} E_{3} \cdots E_{2 f-3} E_{2 f-1} x_{\lambda} T_{d(\mathfrak{s})} T_{w_{p}^{-1}} \\
& =E_{1} E_{3} \cdots E_{2 f-3} x_{\lambda} T_{d(\mathfrak{s})} T_{v} E_{n-2} T_{n-1} T_{n-2}
\end{aligned}
$$

where $v=w_{p}^{-1} s_{n-2} s_{n-1}$ lies in $\mathscr{D}_{f, n-1}$. From the defining relations of $B_{n}(q, r)$,

$$
E_{n-2} T_{n-1} T_{n-2} E_{n-2}=E_{n-2},
$$

and, multiplying both sides of (5.13) on the right by the element $E_{n-2}$,

$$
m_{\lambda} T_{d(\mathfrak{s})} T_{w_{p}^{-1}} E_{n-2}=m_{\lambda} T_{d(\mathfrak{s})} T_{v}, \quad \text { where } v=w_{p}^{-1} s_{n-2} s_{n-1} .
$$

Since $v \in \mathscr{D}_{f, n-1}$, Corollary 5.2 implies a strict inclusion $N^{\mu^{(t)}} \subseteq N^{\mu^{(p)}}$ of $B_{n-1}(q, r)$-modules. 
Recall that if $\lambda$ is a partition of $n-2 f$ and $\mathfrak{s} \in \operatorname{Std}_{n}(\lambda)$, then $\hat{\mathfrak{s}}$ is defined as the standard tableau obtained after relabelling the entries of $\mathfrak{s}$ by $i \mapsto i-2 f$ and $d(\mathfrak{s})$ is the permutation in $\left\langle s_{i}: 2 f<i<n\right\rangle$ defined by the condition that $\mathfrak{s}=\mathfrak{t}^{\lambda} d(\mathfrak{s})$. For the lemmas following, we also recall the definition of the permutation $w_{p}$ in (5.11) above.

Lemma 5.3 Let $f$ be an integer, $0<f \leq[n / 2]$, and $\lambda$ be a partition of $n-2 f$. Suppose that $\mu^{(p)}$ is minimal in $\{v: v \rightarrow \lambda$ and $|v|>|\lambda|\}$ with respect to the dominance order on partitions, let $\mu$ be a partition of $n-2 f+1$ with $\mu \unrhd \mu^{(p)}$ and $\mathfrak{s} \in \operatorname{Std}_{n-1}(\mu)$ be a tableau such that $\mu^{(p)}(\hat{\mathfrak{s}}) \in \mathcal{T}_{0}\left(\mu, \mu^{(p)}\right)$. If $\tau=\operatorname{Shape}\left(\left.\mathfrak{s}\right|_{n-2}\right) \triangleright \lambda$, then

$$
E_{2 f-1} T_{w_{p}}^{-1} T_{d(\mathfrak{s})}^{*} m_{\mu}=E_{1} E_{3} \cdots E_{2 f-1} T_{w_{p}}^{-1} T_{d(\mathfrak{s})}^{*} x_{\mu} \equiv 0 \quad \bmod \check{B}_{n}^{\lambda} .
$$

Proof Recall that $x_{\mu}=\sum_{w \in \mathfrak{S}_{\mu}} q^{\ell(w)} T_{w}$ where $\mathfrak{S}_{\mu}$ is the row stabiliser of $\mathfrak{t}^{\mu} \in$ $\operatorname{Std}_{n-1}(\mu)$ in $\left\langle s_{i}: 2 f-1 \leq i<n-1\right\rangle$. Let

$$
k=\min \left\{i: 2 f-1 \leq i \leq n-2 \text { and }(n-1) d(\mathfrak{s})^{-1} \leq(i) d(\mathfrak{s})^{-1}\right\},
$$

So that

$$
\ell\left(d(\mathfrak{s}) s_{n-2} s_{n-3} \cdots s_{k}\right)=\ell(d(\mathfrak{s}))-n+k+1 .
$$

If we write $v=d(\mathfrak{s}) s_{n-2} s_{n-3} \cdots s_{k}$ and $u=s_{k} s_{k+1} \cdots s_{n-2} w_{p}$, then

$$
\begin{aligned}
E_{2 f-1} T_{w_{p}}^{-1} T_{d(\mathfrak{s})}^{*} m_{\mu} & =E_{2 f-1} T_{w_{p}}^{-1} E_{1} E_{3} \cdots E_{2 f-3} T_{d(\mathfrak{s})}^{*} x_{\mu} \\
& =E_{1} E_{3} \cdots E_{2 f-1} T_{w_{p}}^{-1} T_{d(\mathfrak{s})}^{*} x_{\mu}=E_{1} E_{3} \cdots E_{2 f-1} T_{u}^{-1} T_{v}^{*} x_{\mu} .
\end{aligned}
$$

Since $v$ has a reduced expression $v=s_{i_{1}} s_{i_{2}} \cdots s_{i_{l}}$ in the subgroup $\left\langle s_{i}: 2 f-1 \leq i<\right.$ $n-2\rangle$, we define $v^{\prime}=s_{i_{1}+2} s_{i_{2}+2} \cdots s_{i_{l}+2}$ and, using the braid relation $T_{i}^{-1} T_{i+1}^{-1} T_{i}=$ $T_{i+1} T_{i}^{-1} T_{i+1}^{-1}$, obtain

$$
T_{u}^{-1} T_{i}= \begin{cases}T_{i+2} T_{u}^{-1} & \text { if } 2 f-1 \leq i<k ; \\ T_{i+1} T_{u}^{-1} & \text { if } k<i<n,\end{cases}
$$

which allows us to rewrite (5.14) as

$$
E_{2 f-1} T_{w_{p}}^{-1} T_{d(\mathfrak{s})}^{*} m_{\mu}=E_{1} E_{3} \cdots E_{2 f-1} T_{v^{\prime}}^{*} T_{u}^{-1} x_{\mu} .
$$

Now, to each row $i$ of $\mathfrak{t}^{\mu} \in \operatorname{Std}_{n-1}(\mu)$, associate the subgroup

$$
\mathfrak{R}_{\mathfrak{t}^{\mu}, i}=\left\langle s_{i^{\prime}}: i^{\prime}, i^{\prime}+1 \text { appear in row } i \text { of } \mathfrak{t}^{\mu}\right\rangle
$$

and define $\mathfrak{R}_{\mathfrak{t}^{\tau}, i}$ analogously for $\mathfrak{t}^{\tau} \in \operatorname{Std}_{n}(\tau)$. Let us suppose that $n-1$ appears as an entry in row $j$ of $\mathfrak{s}$; if $i \neq j$, then by (5.15)

$$
\sum_{w \in \Re_{\mathrm{t}} \mu, i} q^{\ell(w)} T_{u}^{-1} T_{w}=\sum_{w \in \mathfrak{R}_{\mathrm{t}^{\tau}, i}} q^{\ell(w)} T_{w} T_{u}^{-1} .
$$


On the other hand, within $\Re_{\mathfrak{t}}{ }^{\mu}, j$ take the parabolic subgroup

$$
\mathfrak{P}_{\mathfrak{t}^{\mu}, j}=\left\langle w \in \mathfrak{R}_{\mathfrak{t}^{\mu}, j}:(k) w=k\right\rangle
$$

and, noting that the set of distinguished right coset representatives for $\mathfrak{P}_{\mathfrak{t}^{\mu}, j}$ in $\mathfrak{R}_{\mathfrak{t}}{ }_{, j}$ (Proposition 3.3 of [8]) is

$$
\mathscr{D}=\left\{v_{i}: v_{0}=1 \text { and } v_{i}=v_{i-1} s_{k-i} \text { for } 0<i \leq \tau_{j}\right\},
$$

we write

$$
\sum_{w \in \Re_{\mathrm{t}^{\mu}, j}} q^{\ell(w)} T_{u}^{-1} T_{w}=\sum_{w \in \mathfrak{P}^{\mu}, j} q^{\ell(w)} T_{u}^{-1} T_{w} \sum_{v \in \mathcal{D}} q^{\ell(v)} T_{v} .
$$

Using the last expression and (5.15), we obtain

$$
T_{u}^{-1} \sum_{w \in \mathfrak{P}_{\mathbf{t}^{\mu}, j}} q^{\ell(w)} T_{w}=\sum_{w \in \mathfrak{R}_{\mathbf{t}^{\tau}, j}} q^{\ell(w)} T_{w} T_{u}^{-1}
$$

which, together with (5.17), implies that

$$
\begin{aligned}
T_{v^{\prime}}^{*} T_{u}^{-1} x_{\mu} & =T_{v^{\prime}}^{*} \sum_{i \geq 1} \sum_{w \in \mathfrak{R}_{\mathfrak{t} \tau}, i} q^{\ell(w)} T_{w} T_{u}^{-1} \sum_{v \in \mathscr{D}} q^{\ell(v)} T_{v} \\
& =T_{v^{\prime}}^{*} x_{\tau} T_{u}^{-1} \sum_{v \in \mathscr{D}} q^{\ell(v)} T_{v} .
\end{aligned}
$$

Since $v^{\prime} \in\left\langle s_{i}: 2 f<i<n\right\rangle$, multiplying both sides of the last expression by $E_{1} E_{3} \cdots E_{2 f-1}$ on the left and referring to (5.16), we obtain

$$
E_{2 f-1} T_{w_{p}}^{-1} T_{d(\mathfrak{s})}^{*} m_{\mu}=T_{v^{\prime}}^{*} E_{1} E_{3} \cdots E_{2 f-1} x_{\tau} T_{u}^{-1} \sum_{v \in \mathscr{D}} q^{\ell(v)} T_{v} .
$$

As the term on the right hand side of the above expression lies in $\check{B}_{n}^{\lambda}$, the result now follows.

The next example illustrates Lemma 5.3.

Example 5.2 In parts (a) and (b) below, let $n=10, f=2$ and $\lambda=(3,2,1)$. Since $\lambda$ has three removable nodes and four addable nodes, the partitions $\mu^{(i)}$ with $\mu^{(i)} \rightarrow \lambda$ and $\left|\mu^{(i)}\right|>|\lambda|$ are

$$
\mu^{(4)}=(4,2,1) \triangleright \mu^{(5)}=(3,3,1) \triangleright \mu^{(6)}=(3,2,2) \triangleright \mu^{(7)}=(3,2,1,1) .
$$

(a) Taking $p=7$, we have $w_{p}=s_{8} s_{7} s_{6} s_{5} s_{4} s_{3} s_{9} s_{8} s_{7} s_{6} s_{5} s_{4}$,

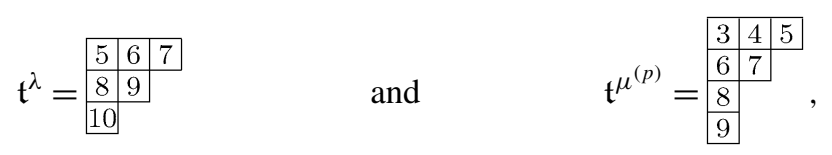


so that $x_{\mu(p)}=\left(1+q T_{3}\right)\left(1+q T_{4}+q^{2} T_{4} T_{3}\right)\left(1+q T_{6}\right)$. Using the braid relation $T_{j}^{-1} T_{j+1}^{-1} T_{j}=T_{j+1} T_{j}^{-1} T_{j+1}^{-1}$, it is verified that

$$
E_{3} T_{w_{p}}^{-1} m_{\mu^{(p)}}=m_{\lambda} T_{w_{p}}^{-1} .
$$

(b) Let $\mu=(4,3)$ and $\mathfrak{s}=$\begin{tabular}{l|l|l|l|l|}
\hline 3 & 4 & 5 & 9 \\
6 & 7 & 8
\end{tabular}$\quad$ so $d(\mathfrak{s})=s_{6} s_{7} s_{8}$. Then

$$
\hat{\mathfrak{s}}=\begin{array}{|l|l|l|l}
1 & 2 & 3 & 7
\end{array} \quad \text { and } \quad \mu^{(p)}(\hat{\mathfrak{s}})=\begin{array}{|l|l|l|l}
\hline 1 & 1 & 1 & 4 \\
\hline 2 & 5 & 6 & 2
\end{array}
$$

as shown in Example 2.2. Now,

$$
6=\min \left\{i \mid 2 f-1 \leq i \leq n-2 \text { and }(n-1) d(\mathfrak{s})^{-1} \leq(i) d(\mathfrak{s})^{-1}\right\},
$$

hence, writing $u=s_{5} s_{4} s_{3} s_{9} s_{8} s_{7} s_{6} s_{5} s_{4}$, one obtains

$$
E_{3} T_{w_{p}}^{-1} T_{d(\mathfrak{s})}^{*} m_{\mu}=E_{3} T_{u}^{-1} m_{\mu}=E_{3} T_{u}^{-1} E_{1} x_{\mu}
$$

where

$$
\begin{aligned}
x_{\mu}=\left(1+q T_{3}\right)\left(1+q T_{4}+q^{2} T_{4} T_{3}\right)\left(1+q T_{5}\right. & \left.+q^{2} T_{5} T_{4}+q^{3} T_{5} T_{4} T_{3}\right) \\
& \times\left(1+q T_{7}\right)\left(1+q T_{8}+q^{2} T_{8} T_{7}\right) .
\end{aligned}
$$

Using the braid relation,

$$
T_{u}^{-1} x_{\mu}=x_{\tau} T_{u}^{-1}\left(1+q T_{5}+q^{2} T_{5} T_{4}+q^{3} T_{5} T_{4} T_{3}\right),
$$

where $\mathfrak{t}^{\tau}=$\begin{tabular}{|l|l|l|}
\hline 5 & 6 & 7 \\
\hline 8 & 9 & 10 \\
\hline
\end{tabular} and

$$
x_{\tau}=\left(1+q T_{5}\right)\left(1+q T_{6}+q^{2} T_{6} T_{5}\right)\left(1+q T_{8}\right)\left(1+q T_{9}+q^{2} T_{9} T_{8}\right) .
$$

As $\tau \triangleright \lambda$, it follows that

$$
\begin{gathered}
E_{3} T_{w_{p}}^{-1} T_{d(\mathfrak{s})}^{*} m_{\mu}=E_{1} E_{3} x_{\tau} T_{u}^{-1}\left(1+q T_{5}+q^{2} T_{5} T_{4}+q^{3} T_{5} T_{4} T_{3}\right) \\
=m_{\tau} T_{u}^{-1}\left(1+q T_{5}+q^{2} T_{5} T_{4}+q^{3} T_{5} T_{4} T_{3}\right) \equiv 0 \bmod \check{B}_{n}^{\lambda} .
\end{gathered}
$$

Corollary 5.4 Let $f$ be an integer $0<f \leq[n / 2]$ and $\lambda$ be a partition of $n-2 f$ with $(p-t)$ addable nodes. Suppose that $\mu^{(1)} \unrhd \mu^{(2)} \unrhd \cdots \unrhd \mu^{(p)}$ is the ordering of $\{\mu: \mu \rightarrow \lambda\}$ by the dominance order on partitions. If $\mu$ is a partition of $n-2 f+1$ such that $\mu \triangleright \mu^{(t+1)}$, and $\mathrm{S} \in \mathcal{T}_{0}\left(\mu, \mu^{(p)}\right)$, then

$$
E_{2 f-1} T_{w_{p}}^{-1} m_{\mathrm{St}} \equiv 0 \quad \bmod B_{n}^{\lambda}, \quad \text { for all } \mathfrak{t} \in \operatorname{Std}_{n-1}(\mu) .
$$

Proof There are $p-t$ standard tableaux $\mathfrak{s}$ labelled by the integers $\{2 f-1,2 f, \ldots$, $n-1\}$ which satisfy the conditions (i) Shape $\left(\left.\mathfrak{s}\right|_{n-2}\right)=\lambda$, and (ii) $\mu^{(p)}(\mathfrak{s}) \in$ $\mathcal{T}_{0}\left(\nu, \mu^{(p)}\right)$, for some partition $\nu$ of $n-2 f+1$; each such tableau $\mathfrak{s}$ additionally 
satisfies the condition that Shape $(\mathfrak{s})=\mu^{(i)}$ for some $i$ with $t<i \leq p$ (the precise form that any such $d(\mathfrak{s})$ must take is given in (5.19) below). Thus if $\mu$ is as given in the statement of the corollary and $\mathfrak{s} \in \operatorname{Std}_{n-1}(\mu)$ satisfies $\mu^{(p)}(\hat{\mathfrak{s}}) \in \mathcal{T}_{0}\left(\mu, \mu^{(p)}\right)$, then $\tau=\operatorname{Shape}\left(\left.\mathfrak{s}\right|_{n-2}\right) \triangleright \lambda$, so by Lemma 5.3,

$$
E_{2 f-1} T_{w_{p}}^{-1} T_{d(\mathfrak{s})}^{*} m_{\mu} \equiv 0 \quad \bmod B_{n}^{\lambda} .
$$

Using the definition of $m_{\mathrm{St}}$, the result now follows.

Lemma 5.5 Let $f$ be an integer, $0<f \leq[n / 2]$, and $\lambda \vdash n-2 f, \mu \vdash n-2 f+1$ be partitions such that $\mu \rightarrow \lambda$. If $\mu^{(p)}$ is minimal with respect to dominance order among $\{v: v \rightarrow \lambda$ and $|v|>|\lambda|\}$, and $\mathfrak{s} \in \operatorname{Std}_{n-1}(\mu)$ is a tableau such that $\mu^{(p)}(\hat{\mathfrak{s}}) \in$ $\mathcal{T}_{0}\left(\mu, \mu^{(p)}\right)$, then there exist $a_{(\mathfrak{t}, w)} \in R$, for $(\mathfrak{t}, w) \in \mathcal{I}_{n}(\lambda)$, such that

$$
E_{2 f-1} T_{w_{p}}^{-1} T_{d(\mathfrak{s})}^{*} m_{\mu} \equiv \sum_{(\mathfrak{t}, w) \in \mathcal{I}_{n}(\lambda)} a_{(\mathfrak{t}, w)} m_{\lambda} T_{d(\mathfrak{t})} T_{w} \quad \bmod \check{B}_{n}^{\lambda} .
$$

Proof There is a unique tableau $\mathfrak{s} \in \operatorname{Std}_{n-1}(\mu)$ satisfying the hypotheses of the lemma, namely the tableau with $\left.\mathfrak{s}\right|_{n-1}=\mathfrak{t}^{\lambda} \in \operatorname{Std}_{n-2}(\lambda)$. Furthermore,

$$
d(\mathfrak{s})=s_{k} s_{k+1} \cdots s_{n-2} \quad \text { where } \quad k=(n-1) d(\mathfrak{s})^{-1} .
$$

Suppose that $k$ appears as an entry in the row $j$ of $\mathfrak{s}$. As in the proof of Lemma 5.3, we associate to row $j$ of $\mathfrak{t}^{\mu}$ the subgroup

$$
\mathfrak{R}_{\mathfrak{t}}{ }^{\mu}, j=\left\langle s_{i}: i, i+1 \text { appear in row } j \text { of } \mathfrak{t}^{\mu}\right\rangle
$$

and take the parabolic subgroup $\mathfrak{P}_{\mathfrak{t}^{\mu}, j}=\left\langle w \in \mathfrak{R}_{\mathfrak{t} \mu, j}:(k) w=k\right\rangle \subseteq \mathfrak{R}_{\mathfrak{t}^{\mu}, j}$. The set of distinguished right coset representatives for $\mathfrak{P}_{\mathfrak{t}^{\mu}, j}$ in $\mathfrak{R}_{\mathfrak{t}^{\mu}, j}$ is

$$
\mathscr{D}=\left\{v_{i}: v_{0}=1 \text { and } v_{i}=v_{i-1} s_{k-i} \text { for } 0<i \leq \lambda_{j}\right\} .
$$

As in the proof of Lemma 5.3, the coset representatives $\mathcal{D}$ enable us to write

$$
E_{2 f-1} T_{w_{p}}^{-1} T_{d(\mathfrak{s})}^{*} m_{\mu}=m_{\lambda} T_{u}^{-1} \sum_{v \in \mathscr{D}} q^{\ell(v)} T_{v}
$$

where $u=s_{k} s_{k+1} \cdots s_{n-2} w_{p}=s_{k-1} s_{k-2} \cdots s_{2 f-1} s_{n-1} s_{n-2} \cdots s_{2 f}$.

Let $f$ be an integer, $0 \leq f \leq[n / 2]$, and $\lambda$ be a partition of $n-2 f$ with $t$ removable and $p-t$ addable nodes. Take $\mu^{(t+1)} \triangleright \mu^{(t+2)} \triangleright \cdots \triangleright \mu^{(p)}$ as the ordering of the set $\{\mu: \mu \rightarrow \lambda$ and $|\mu|>|\lambda|\}$ by dominance order on partitions and, for $t<k \leq p$, suppose that $[\lambda]$ is the diagram obtained by deleting a node from the row $j_{k}$ of $\left[\mu^{(k)}\right]$. There exists for each $\mu^{(k)}$ with $\mu^{(k)} \rightarrow \lambda$ and $\left|\mu^{(k)}\right|>|\lambda|$, a unique tableau $\mathfrak{s}_{k} \in$ $\operatorname{Std}_{n-1}\left(\mu^{(k)}\right)$ such that $\mu^{(p)}\left(\mathfrak{s}_{k}\right) \in \mathcal{T}_{0}\left(\mu^{(k)}, \mu^{(p)}\right)$ and Shape $\left(\left.\mathfrak{s}_{k}\right|_{n-2}\right)=\lambda$. To wit, $\mathfrak{s}_{k}$ is determined by

$$
d\left(\mathfrak{s}_{k}\right)=s_{a_{k}} s_{a_{k}+1} \cdots s_{n-2} \quad \text { where } \quad a_{k}=2(f-1)+\sum_{i=1}^{j_{k}} \mu_{i}^{(k)} .
$$


Thus we let

$$
w_{k}=d\left(\mathfrak{s}_{k}\right)^{-1} w_{p}=s_{a_{k}-1} s_{a_{k}-2} \cdots s_{2 f-1} s_{n-1} s_{n-2} \cdots s_{2 f},
$$

and write

$$
y_{\mu^{(k)}}^{\lambda}=E_{2 f-1} T_{w_{k}}^{-1} m_{\mu^{(k)}}+\check{B}_{n}^{\lambda} .
$$

By Lemma 5.5, we note that $y_{\mu^{(k)}}^{\lambda}$ is a well defined element in the $B_{n}(q, r)$-module $S^{\lambda}$. We define $N^{\mu^{(k)}}$, for $t<k \leq p$, to be the $B_{n-1}(q, r)$-submodule of $S^{\lambda}$ generated by $y_{\mu^{(k)}}^{\lambda}$.

Example 5.3 Let $n=4, f=1$. If $\lambda=(1,1)$, and $\mu=(2,1)$, then $\mathfrak{s}=$\begin{tabular}{|l|l|}
\hline & 3 \\
2
\end{tabular} is the unique tableau with $\left.\mathfrak{s}\right|_{n-1}=\mathfrak{t}^{\lambda} \in \operatorname{Std}_{n-2}(\lambda)$. Thus $y_{\mu}^{\lambda}=E_{1} T_{w_{p}}^{-1} T_{d(\mathfrak{s})}^{*} m_{\mu}+\check{B}_{4}^{\lambda}=$ $E_{1} T_{2}^{-1} T_{1}^{-1} T_{3}^{-1}\left(1+q T_{1}\right)+\check{B}_{4}^{\lambda}$.

Recall that $N^{\mu^{(t)}} \subseteq N^{\mu^{(p)}}$ is a strict inclusion of $B_{n-1}(q, r)$-modules.

Lemma 5.6 Let $f$ be an integer, $0<f \leq[n / 2]$, and $\lambda$ be a partition of $n-2 f$ with $t$ removable nodes and $(p-t)$ addable nodes. Suppose that $\mu^{(t+1)} \triangleright \mu^{(t+2)} \triangleright \cdots \triangleright \mu^{(p)}$ $i$ the ordering of $\{\mu: \mu \rightarrow \lambda$ and $|\mu|>|\lambda|\}$ by dominance order on partitions. Then the right $B_{n-1}(q, r)$-module $N^{\mu^{(p)}} / N^{\mu^{(t)}}$ is generated as an $R$-module by

$$
\left\{y_{\mu^{(k)}}^{\lambda} T_{d(\mathfrak{t})} T_{w}+N^{\mu^{(t)}}:(\mathfrak{t}, w) \in \mathcal{I}_{n-1}\left(\mu^{(k)}\right) \text { and } t<k \leq p\right\} .
$$

Proof From the expression (5.21), observe that the $B_{n-1}(q, r)-$ module $N^{\mu^{(p)}}$ is generated as an $R$-module by elements of the form

$$
y_{\mu^{(p)}}^{\lambda} b=m_{\lambda} T_{w_{p}}^{-1} b+\check{B}_{n}^{\lambda}=E_{2 f-1} T_{w_{p}}^{-1} m_{\mu^{(p)}} b+\check{B}_{n}^{\lambda}, \quad \text { for } b \in B_{n-1}(q, r) .
$$

Let $b \in B_{n-1}(q, r)$. Then, by Lemma 3.6, there exist $\mathrm{S} \in \mathcal{T}_{0}\left(\mu, \mu^{(p)}\right)$, for $\mu \unrhd \mu^{(p)}$ and $|\mu|=\left|\mu^{(p)}\right|$, together and $a_{\mathrm{S}, \mathfrak{t}, w}$, for $(\mathfrak{t}, w) \in \mathcal{I}_{n-1}(\mu)$, such that

$$
m_{\mu^{(p)}} b=\sum_{\substack{\mu \unrhd \mu^{(p)} \\(\mathfrak{t}, w) \in \mathcal{I}_{n-1}(\mu) \\ \mathbf{S} \in \mathcal{T}_{0}\left(\mu, \mu^{(p)}\right)}} a_{\mathrm{S}, \mathfrak{t}, w} m_{\mathrm{St}} T_{w}+b^{\prime},
$$

where $b^{\prime} \in B_{n-1}^{f}$. Since the process of rewriting a product

$$
E_{1} E_{3} \cdots E_{2 f-3} T_{u} T_{v} b, \quad \text { for } u \in\left\langle s_{i}: 2 f-2<i<n-1\right\rangle, v \in \mathcal{D}_{f-1, n-1},
$$

in terms of the basis (3.5) depends only on (3.12), Proposition 3.7 of [4] and operations in the subalgebra $\left\langle T_{i}: 2 f-2<i<n-1\right\rangle \subseteq B_{n-1}(q, r)$, we note that the term $b^{\prime}$ in (5.22) satisfies

$$
b^{\prime} \in\left(E_{1} E_{3} \cdots E_{2 f-3}\right) B_{n-1}(q, r) \cap B_{n-1}^{f} .
$$


By decomposing the set $\left\{\mu:|\mu|=n-2 f+1\right.$ and $\left.\mu \unrhd \mu^{(p)}\right\}$ and using Lemma 5.3, we obtain, for each $w \in \mathscr{D}_{f-1, n-1}$, an expression:

$$
\sum_{\substack{\mu \unrhd \mu^{(p)} \\ \mathfrak{t} \in \operatorname{Std}_{n-1}(\mu) \\ \mathbf{S} \in \mathcal{T}_{0}\left(\mu, \mu^{(p)}\right)}} a_{\mathrm{S}, \mathfrak{t}, w} m_{\mathrm{St}} T_{w}=\sum_{\substack{t<k \leq p \\ \mathfrak{t} \in \operatorname{Std}_{n-1}\left(\mu^{(k)}\right) \\ \mathbf{S} \in \mathcal{T}_{0}\left(\mu^{(k)}, \mu^{(p)}\right)}} a_{\mathrm{S}, \mathfrak{t}, w} m_{\mathrm{St}} T_{w}+\sum_{\substack{\mu \triangleright \mu^{(t+1)} \\ \mathfrak{t} \in \operatorname{Std}_{n-1}(\mu) \\ \mathbf{S} \in \mathcal{T}_{0}\left(\mu, \mu^{(p)}\right)}} a_{\mathrm{S}, \mathfrak{t}, w} m_{\mathrm{St}} T_{w} .
$$

Hence, multiplying both sides of (5.22) by $E_{2 f-1} T_{w_{p}}^{-1}$ on the left, and using (5.23) together with Corollary 5.4, we obtain:

$$
\begin{aligned}
& E_{2 f-1} T_{w_{p}}^{-1} m_{\mu^{(p)}} b+\check{B}_{n}^{\lambda} \\
& =E_{2 f-1} T_{w_{p}}^{-1} \sum_{\substack{t<k \leq p \\
(\mathfrak{t}, w) \in \mathcal{I}_{n-1}\left(\mu^{(k)}\right) \\
\mathrm{S} \in \mathcal{T}_{0}\left(\mu^{(k)}, \mu^{(p)}\right)}} a_{\mathrm{S}, \mathfrak{t}, w} m_{\mathrm{St}} T_{w}+E_{2 f-1} T_{w_{p}}^{-1} b^{\prime}+\check{B}_{n}^{\lambda} .
\end{aligned}
$$

We recall the definition of the tableaux $\mathfrak{s}_{k} \in \operatorname{Std}_{n-1}\left(\mu^{(k)}\right)$, for $t<k \leq p$, in (5.19), and also that the $w_{k}$ defined, for $t<k \leq p$, by (5.20), are chosen so that $T_{w_{p}}^{-1} T_{d\left(\mathfrak{s}_{k}\right)}^{*}=$ $T_{w_{k}}^{-1}$. Thus

$$
\begin{aligned}
& E_{2 f-1} T_{w_{p}}^{-1} m_{\mu^{(p)}} b+\check{B}_{n}^{\lambda} \\
& =\sum_{\substack{t<k \leq p \\
(\mathfrak{t}, w) \in \mathcal{I}_{n-1}\left(\mu^{(k)}\right)}} a_{k, \mathfrak{t}, w} E_{2 f-1} T_{w_{k}}^{-1} m_{\mu^{(k)}} T_{d(\mathfrak{t})} T_{w}+E_{2 f-1} T_{w_{p}}^{-1} b^{\prime}+\check{B}_{n}^{\lambda},
\end{aligned}
$$

where $a_{k, \mathfrak{t}, w}=q^{\ell\left(d\left(\mathfrak{s}_{k}\right)\right)} a_{\mathrm{S}, \mathfrak{t}, w}$ whenever $\mu^{(p)}\left(\hat{\mathfrak{s}}_{k}\right)=\mathrm{S}$. Thus we have shown that

$$
E_{2 f-1} T_{w_{p}}^{-1} m_{\mu^{(p)}} b+\check{B}_{n}^{\lambda}=\sum_{\substack{t<k \leq p \\(\mathfrak{t}, w) \in \mathcal{I}_{n-1}\left(\mu^{(k)}\right)}} a_{k, \mathfrak{t}, w} y_{\mu^{(k)}}^{\lambda} T_{d(\mathfrak{t})} T_{w}+E_{2 f-1} T_{w_{p}}^{-1} b^{\prime}+\check{B}_{n}^{\lambda} .
$$

It now remains to show that $E_{2 f-1} T_{w_{p}}^{-1} b^{\prime}+\check{B}_{n}^{\lambda} \in N^{\mu^{(t)}}$. Noting the characterisation of the $B_{n-1}(q, r)$-module $N^{\mu^{(t)}}$ given in Corollary 5.2, to complete the proof of the lemma, it suffices to demonstrate the statement following.

Claim 5.7 If $b \in\left(E_{1} E_{3} \cdots E_{2 f-3}\right) B_{n-1}(q, r) \cap B_{n-1}^{f}$ then there exist $a_{\mathfrak{s}, \mathfrak{t}, w} \in R$, for $\mathfrak{s}, \mathfrak{t} \in \operatorname{Std}_{n}(v), w \in \mathscr{D}_{f, n-1}$ and $v \vdash n-2 f$, such that

$$
E_{2 f-1} T_{w_{p}}^{-1} b \equiv \sum_{\substack{\nu \vdash n-2 f \\ \mathfrak{s}, \mathfrak{t} \in \operatorname{Std}_{n}(v) \\ w \in \mathcal{D}_{f, n-1}}} a_{\mathfrak{s}, \mathfrak{t}, w} T_{d(\mathfrak{s})}^{*} m_{\nu} T_{d(\mathfrak{t})} T_{w} \quad \bmod B_{n}^{f+1}
$$


We now prove the claim. Let $b \in\left(E_{1} E_{3} \cdots E_{2 f-3}\right) B_{n-1}(q, r) \cap B_{n-1}^{f}$. As in the proof of Lemma 3.5, we may write $b$, modulo $B_{n-1}^{f+1} \subset B_{n}^{f+1}$, as an $R$-linear combination of elements of the form

$$
\left\{T_{v}^{*} E_{1} E_{3} \cdots E_{2 f-1} T_{u} T_{w} \mid \begin{array}{c}
v, w \in \mathcal{D}_{f, n-1}, u \in\left\langle s_{i}: 2 f<i<n-1\right\rangle \\
\text { and } v \in\left\langle s_{i}: 2 f-2<i<n-1\right\rangle
\end{array}\right\} .
$$

Multiplying an element of the above set on the left by $E_{2 f-1} T_{w_{p}}^{-1}$, we obtain:

$$
E_{1} E_{3} \cdots E_{2 f-3} E_{2 f-1} T_{w_{p}}^{-1} T_{v}^{*} E_{2 f-1} T_{u} T_{w} .
$$

There are two cases following. In the first case, suppose that $v$ has a reduced expression $v=s_{i_{1}} s_{i_{2}} \cdots s_{i_{l}}$ in $\left\langle s_{i}: 2 f-2<i<n-2\right\rangle$. Applying the relations

$$
T_{i}^{-1} T_{i+1}^{-1} T_{i}=T_{i+1} T_{i}^{-1} T_{i+1}^{-1} \quad \text { and } \quad T_{i}^{-1} T_{i+1}^{-1} E_{i}=E_{i+1} T_{i}^{-1} T_{i+1}^{-1},
$$

we obtain $T_{w_{p}}^{-1} T_{v}^{*} E_{2 f-1}=T_{v^{\prime \prime}}^{*} E_{2 f+1} T_{w_{p}}^{-1}$, where $v^{\prime \prime}=s_{i_{1}+2} s_{i_{2}+2} \cdots s_{i_{l}+2}$. As $T_{v^{\prime \prime}}^{*}$ commutes with $E_{1} E_{3} \cdots E_{2 f-1}$, substitution into (5.26) yields:

$$
E_{1} E_{3} \cdots E_{2 f-1} T_{w_{p}}^{-1} T_{v}^{*} E_{2 f-1} T_{u} T_{w}=T_{v^{\prime \prime}}^{*} E_{1} E_{3} \cdots E_{2 f+1} T_{w_{p}}^{-1} T_{u} T_{w}
$$

which is visibly a term in $B_{n}^{f+1}$.

In the second case, suppose that $v$ does not have a reduced expression in $\left\langle s_{i}\right.$ : $2 f-2<i<n-2\rangle$. To obtain an explicit expression for such $v$, we first enumerate the elements of

$$
\mathcal{D}_{f, n-1} \cap\left\langle s_{i}: 2 f-2<i<n-1\right\rangle .
$$

As in Example 3.2, the elements of the set (5.27) take the form

$$
v_{i, j}=s_{2 f} s_{2 f+1} \cdots s_{j-1} s_{2 f-1} s_{2 f} \cdots s_{i-1}, \quad \text { for } 2 f-2<i<j<n .
$$

Now, $v_{i, j}$ does not have a reduced expression in $\left\langle s_{i}: 2 f-2<i<n-2\right\rangle$ if and only if $v_{i, j}$ does not stabilise $n-1$; thus $v_{i, j}=v_{i, n-1}$, for some $2 f-2<i<n-1$. Define

$$
v_{i}=v_{i, n-1}=s_{2 f} s_{2 f+1} \cdots s_{n-2} s_{2 f-1} s_{2 f} \cdots s_{i-1}, \quad \text { for } 2 f-2<i<n-1,
$$

so the elements of the set (5.27) which do not stabilise $n-1$ are precisely

$$
\left\{v_{i}: 2 f-1 \leq i \leq n-2\right\} .
$$

Let $j$ be an integer, $2 f-1 \leq j \leq n-2$, and calculate $E_{2 f-1} T_{w_{p}}^{-1} T_{v_{j}}^{*} E_{2 f-1}$ explicitly, beginning with:

$$
\begin{aligned}
& E_{2 f-1} T_{w_{p}}^{-1} T_{v_{j}}^{*} E_{2 f-1} \\
& \quad=E_{2 f-1} T_{w_{p}}^{-1}\left(T_{j-1} T_{j-2} \cdots T_{2 f-1}\right)\left(T_{n-2} T_{n-3} \cdots T_{2 f}\right) E_{2 f-1} \\
& \quad=E_{2 f-1} T_{w_{p}}^{-1}\left(T_{n-2} T_{n-3} \cdots T_{j+1}\right)\left(T_{j-1} T_{j-2} \cdots T_{2 f-1}\right)\left(T_{j} T_{j-1} \cdots T_{2 f}\right) E_{2 f-1} \\
& \quad=E_{2 f-1}\left(T_{2 f}^{-1} T_{2 f+1}^{-1} \cdots T_{n-1}^{-1}\right)
\end{aligned}
$$




$$
\begin{aligned}
& \times\left(T_{2 f-1}^{-1} T_{2 f}^{-1} \cdots T_{j}^{-1}\right)\left(T_{j-1} T_{j-2} \cdots T_{2 f-1}\right)\left(T_{j} T_{j-1} \cdots T_{2 f}\right) E_{2 f-1} \\
= & E_{2 f-1}\left(T_{2 f}^{-1} T_{2 f+1}^{-1} \cdots T_{j+1}^{-1}\right)\left(T_{2 f-1}^{-1} T_{2 f}^{-1} \cdots T_{j}^{-1}\right)\left(T_{j-1} T_{j-2} \cdots T_{2 f-1}\right) \\
& \times\left(T_{j} T_{j-1} \cdots T_{2 f}\right) E_{2 f-1}\left(T_{j+2}^{-1} T_{j+3}^{-1} \cdots T_{n-1}^{-1}\right) .
\end{aligned}
$$

Using the relations

$$
E_{2 f-1}\left(T_{2 f}^{-1} T_{2 f+1}^{-1} \cdots T_{j+1}^{-1}\right)\left(T_{2 f-1}^{-1} T_{2 f}^{-1} \cdots T_{j}^{-1}\right)=E_{2 f-1} E_{2 f} \cdots E_{j+1}
$$

and

$$
\left(T_{j-1} T_{j-2} \cdots T_{2 f-1}\right)\left(T_{j} T_{j-1} \cdots T_{2 f}\right) E_{2 f-1}=E_{j} E_{j-1} \cdots E_{2 f-1},
$$

we now obtain:

$$
\begin{aligned}
E_{2 f-1} T_{w_{p}}^{-1} T_{v_{j}}^{*} E_{2 f-1}= & \left(E_{2 f-1} E_{2 f} \cdots E_{j} E_{j+1}\right)\left(E_{j} E_{j-1} \cdots E_{2 f-1}\right) \\
& \times\left(T_{j+2}^{-1} T_{j+3}^{-1} \cdots T_{n-1}^{-1}\right)
\end{aligned}
$$

Further applying relations like $E_{i}\left(E_{i+1} E_{i+2} E_{i+1}\right) E_{i}=E_{i} E_{i+1} E_{i}=E_{i}$ in the right hand side of the above expression gives:

$$
E_{2 f-1} T_{w_{p}}^{-1} T_{v_{j}}^{*} E_{2 f-1}=E_{2 f-1}\left(T_{j+2}^{-1} T_{j+3}^{-1} \cdots T_{n-1}^{-1}\right) .
$$

Multiplying both sides of (5.28) by $E_{1} E_{3} \cdots E_{2 f-3}$ on the left and by $T_{u} T_{w}$ on the right, the term (5.26), with $v_{j}$ substituted for $v$, becomes

$$
E_{1} E_{3} \cdots E_{2 f-1} T_{w_{p}}^{-1} T_{v_{j}}^{*} E_{2 f-1} T_{u} T_{w}=E_{1} E_{3} \cdots E_{2 f-1}\left(T_{j+2}^{-1} T_{j+3}^{-1} \cdots T_{n-1}^{-1}\right) T_{u} T_{w} .
$$

Now $\left(T_{j+2}^{-1} T_{j+3}^{-1} \cdots T_{n-1}^{-1}\right) T_{u}$ lies in $\left\langle T_{2 f+1}, T_{2 f+2}, \ldots, T_{n-1}\right\rangle \subseteq B_{n}(q, r)$ and consequently, using Theorem 3.1, can be expressed as an $R$-linear sum of elements from the set $\left\{T_{u^{\prime}}: u^{\prime} \in\left\langle s_{i}: 2 f<i<n\right\rangle\right\}$ together with an element $b^{\prime}$ from the two-sided ideal of $\left\langle T_{2 f+1}, T_{2 f+2}, \ldots, T_{n-1}\right\rangle$ generated by $E_{2 f+1}$. By Lemma 3.3, the element labelled $b^{\prime}$ immediately preceding satisfies

$$
E_{1} E_{3} \cdots E_{2 f-1} E_{2 f-1} b^{\prime} T_{w} \in B_{n}^{f+1},
$$

and can be safely ignored in any calculation modulo $\check{B}_{n}^{\lambda}$. If $w \in \mathscr{D}_{f, n-1}$, then straightening a term

$$
E_{1} E_{3} \cdots E_{2 f-1} T_{u^{\prime}} T_{w}, \quad \text { for } u^{\prime} \in\left\langle s_{i}: 2 f<i<n\right\rangle,
$$

into linear combinations of the basis elements given in Theorem 3.1, is achieved using relations in $\mathscr{H}_{n-2 f}\left(q^{2}\right)$, via the map $\vartheta_{f}$, and does not involve any transformation of $T_{w}$; it follows that there exist $a_{\mathfrak{u}, \mathfrak{v}, w}$, for $\mathfrak{u}, \mathfrak{v} \in \operatorname{Std}_{n}(v)$ and $v \vdash n-2 f$, such that the term (5.29) can be expressed as

$$
E_{1} E_{3} \cdots E_{2 f-1} T_{u^{\prime}} T_{w} \equiv \sum_{\substack{\nu \vdash n-2 f \\ \mathfrak{u}, \mathfrak{v} \in \operatorname{Std}_{n}(v)}} a_{\mathfrak{u}, \mathfrak{v}, w} T_{d(\mathfrak{u})}^{*} m_{v} T_{d(\mathfrak{v})} T_{w} \quad \bmod B_{n}^{f+1} .
$$


This completes the proof of the claim.

We continue to use the notation established in the statement of Lemma 5.6.

If $t<k \leq p$, then by Lemma 5.6, there is a proper inclusion of $B_{n-1}(q, r)-$ modules $N^{\mu^{(t)}} \subseteq N^{\mu^{(k)}}$.

Corollary 5.8 Let $f$ be an integer, $0<f \leq[n / 2]$, and $\lambda$ be a partition of $n-2 f$ with $t$ removable nodes and $(p-t)$ addable nodes. Suppose that $\mu^{(1)} \triangleright \mu^{(2)} \triangleright \cdots \triangleright \mu^{(p)}$ is the ordering of $\{\mu: \mu \rightarrow \lambda\}$ by dominance order on partitions. Then

$$
(0)=N^{\mu^{(0)}} \subseteq N^{\mu^{(1)}} \subseteq \cdots \subseteq N^{\mu^{(p)}}=\operatorname{Res}\left(S^{\lambda}\right)
$$

is a filtration of $\operatorname{Res}\left(S^{\lambda}\right)$ by $B_{n-1}(q, r)$-modules, wherein each quotient $N^{\mu^{(k)}} / N^{\mu^{(k-1)}}$, for $1 \leq k \leq p$, is isomorphic to the cell module $S^{\mu^{(k)}}$ via

$$
y_{\mu^{(k)}}^{\lambda} T_{d(\mathfrak{t})} T_{w}+N^{\mu^{(k-1)}} \mapsto m_{\mu^{(k)}} T_{d(\mathfrak{t})} T_{w}+\check{B}_{n-1}^{\mu^{(k)}}
$$

for $(\mathfrak{t}, w) \in \operatorname{Std}_{n-1}\left(\mu^{(k)}\right)$.

Proof It has been shown in Lemma 5.1 that the map (5.30) is an isomorphism $N^{\mu^{(k)}} / N^{\mu^{(k-1)}} \cong S^{\mu^{(k)}}$, for $1 \leq k \leq t$.

For each $k$ with $t<k \leq p$, let $\mathrm{S}_{k}=\mu^{(p)}\left(\mathfrak{s}_{k}\right)$, where $\mathfrak{s}_{k}$ is the tableau defined by (5.19). If $\mathfrak{v} \in \operatorname{Std}_{n-1}\left(\mu^{(k)}\right)$ and $b \in B_{n-1}(q, r)$, then using Lemmas 3.6 and 5.3, there exist $a_{j, \mathfrak{t}, w} \in R$, for $(\mathfrak{t}, w) \in \mathcal{I}_{n-1}\left(\mu^{(j)}\right)$, and $t<j \leq k$, such that

$$
m_{\mathrm{S}_{k} \mathfrak{v}} b=\sum_{\substack{t<j \leq k \\
(\mathfrak{t}, w) \in \mathcal{I}_{n-1}\left(\mu^{(j)}\right)}} a_{j, \mathfrak{t}, w} m_{\mathrm{S}_{j} \mathfrak{t}} T_{w}+\sum_{\substack { \mu \triangleright \mu^{(t+1)} \\
\begin{subarray}{c}{\mathrm{~S} \in \mathcal{T}_{0}\left(\mu, \mu \\
(\mathfrak{u}, v) \in \mathcal{I}_{n-1}(\mu)\right.{ \mu \triangleright \mu ^ { ( t + 1 ) } \\
\begin{subarray} { c } { \mathrm { S } \in \mathcal { T } _ { 0 } ( \mu , \mu \\
( \mathfrak { u } , v ) \in \mathcal { I } _ { n - 1 } ( \mu ) } }\end{subarray}} a_{\mathrm{S}, \mathfrak{u}, v} m_{\mathrm{Su}_{\mathfrak{u}}} T_{v}+b^{\prime}
$$

where $\mu$ runs over partitions of $n-2 f+1$ and

$$
b^{\prime} \in E_{1} E_{3} \cdots E_{2 f-3} B_{n-1}(q, r) \cap B_{n-1}^{f} .
$$

Multiplying both sides of the expression (5.31) by $E_{2 f-1} T_{w_{p}}^{-1}$ and using Lemma 5.3, we obtain

$$
\begin{aligned}
q^{\ell\left(d\left(\mathfrak{s}_{k}\right)\right)} y_{\mu^{(k)}}^{\lambda} T_{d(\mathfrak{v})} b= & E_{2 f-1} T_{w_{k}}^{-1} m_{\mu^{(k)}} b+\check{B}_{n}^{\lambda} \\
= & \sum_{\substack{t<j \leq k \\
(\mathfrak{t}, w) \in \mathcal{I}_{n-1}\left(\mu^{(j)}\right)}} a_{j, \mathfrak{t}, w} q^{\ell\left(d\left(\mathfrak{s}_{j}\right)\right)} y_{\mu(j)}^{\lambda} T_{d(\mathfrak{t})} T_{w} \\
& +E_{2 f-1} T_{w_{p}}^{-1} b^{\prime}+\check{B}_{n}^{\lambda}
\end{aligned}
$$


where $E_{2 f-1} T_{w_{p}}^{-1} b^{\prime}+\check{B}_{n}^{\lambda} \in N^{\mu^{(t)}}$ by Claim 5.7. Thus

$$
q^{\ell\left(d\left(\mathfrak{s}_{k}\right)\right)} y_{\mu^{(k)}}^{\lambda} T_{d(\mathfrak{v})} b \equiv \sum_{\substack{t<j \leq k \\(\mathfrak{t}, w) \in \mathcal{I}_{n-1}\left(\mu^{(j)}\right)}} a_{j, \mathfrak{t}, w} q^{\ell\left(d\left(\mathfrak{s}_{j}\right)\right)} y_{\mu}^{\lambda}\left({ }^{(j)} T_{d(\mathfrak{t})} T_{w} \quad \bmod N^{\mu^{(t)}}\right.
$$

and

$$
\begin{aligned}
q^{\ell\left(d\left(\mathfrak{s}_{k}\right)\right)} y_{\mu^{(k)}}^{\lambda} T_{d(\mathfrak{v})} b \equiv & \sum_{\substack{(\mathfrak{t}, w) \in \mathcal{I}_{n-1}\left(\mu^{(k)}\right)\\
}} a_{k, \mathfrak{t}, w} q^{\ell\left(d\left(\mathfrak{s}_{k}\right)\right)} y_{\mu^{(k)}}^{\lambda} T_{d(\mathfrak{t})} T_{w} \\
& +\sum_{\substack{t<j<k \\
(\mathfrak{t}, w) \in \mathcal{I}_{n-1}\left(\mu^{(j)}\right)}} a_{j, \mathfrak{t}, w} q^{\ell\left(d\left(\mathfrak{s}_{j}\right)\right)} y_{\mu^{(j)}}^{\lambda} T_{d(\mathfrak{t})} T_{w} \bmod N^{\mu^{(t)}} .
\end{aligned}
$$

From (3.16) and (5.31), the $\left\{a_{k, \mathfrak{t}, w} \in R:(\mathfrak{t}, w) \in \mathcal{I}_{n-1}\left(\mu^{(k)}\right)\right\}$ appearing in (5.32) satisfy $a_{k, \mathfrak{t}, w}=a_{\mathfrak{t}, w}$, where

$$
m_{\mu^{(k)}} T_{d(\mathfrak{v})} b \equiv \sum_{(\mathfrak{t}, w) \in \mathcal{I}_{n-1}\left(\mu^{(k)}\right)} a_{\mathfrak{t}, w} m_{\mu^{(k)}} T_{d(\mathfrak{t})} T_{w} \quad \bmod \check{B}_{n-1}^{\mu^{(k)}},
$$

thus demonstrating that (5.30) determines a $B_{n-1}(q, r)$-module isomorphism whenever $t<k \leq p$.

It remains to observe that $N^{\mu^{(p)}}=\operatorname{Res}\left(S^{\lambda}\right)$. To this end,

$$
\operatorname{dim}_{R}\left(N^{\mu^{(p)}}\right)=\sum_{i=1}^{p} \operatorname{dim}_{R}\left(N^{\mu^{(i)}} / N^{\mu^{(i-1)}}\right)=\sum_{\mu \rightarrow \lambda} \operatorname{dim}_{R}\left(S^{\mu}\right)=\operatorname{dim}_{R}\left(S^{\lambda}\right)
$$

where the last equality follows, for instance, from the semisimple branching law given in Theorem 2.3 of [12].

The statement below follows from Corollary 5.8.

Theorem 5.9 Let $f$ be an integer, $0 \leq f \leq[n / 2]$, and $\lambda$ be a partition of $n-2 f$. Suppose that for each partition $\mu$ with $\mu \rightarrow \lambda$ there exists an index set $\mathfrak{T}_{n-1}(\mu)$ together with

$$
\left\{b_{\mathfrak{u}} \in B_{n-1}(q, r): \mathfrak{u} \in \mathfrak{T}_{n-1}(\mu)\right\}
$$

such that

$$
\left\{m_{\mathfrak{u}}=m_{\mu} b_{\mathfrak{u}}+\check{B}_{n-1}^{\mu}: \mathfrak{u} \in \mathfrak{T}_{n-1}(\mu)\right\}
$$

freely generates $S^{\mu}$ as an $R$-module. Then

$$
\left\{y_{\mu}^{\lambda} b_{\mathfrak{u}}: \mathfrak{u} \in \mathfrak{T}_{n-1}(\mu) \text { for } \mu \rightarrow \lambda\right\}
$$


is a free $R$-basis for $S^{\lambda}$. Moreover, if $\check{N}^{\mu}$ denotes the $B_{n-1}(q, r)$-submodule of $S^{\lambda}$ generated by

$$
\left\{y_{\nu}^{\lambda} b_{\mathfrak{t}}: \mathfrak{t} \in \mathfrak{T}_{n-1}(v) \text { for } v \rightarrow \lambda \text { and } v \triangleright \mu\right\},
$$

then

$$
y_{\mu}^{\lambda} b_{\mathfrak{u}}+\check{N}^{\mu} \mapsto m_{\mu} b_{\mathfrak{u}}+\check{B}_{n-1}^{\mu} \quad \text { for } \mathfrak{u} \in \mathfrak{T}_{n-1}(\mu) \text { with } \mu \rightarrow \lambda
$$

determines an isomorphism $N^{\mu} / \check{N}^{\mu} \cong S^{\mu}$ of $B_{n-1}(q, r)$-modules.

\section{New bases for the B-M-W algebras}

If $f$ is an integer, $0 \leq f \leq[n / 2]$, and $\lambda$ is a partition of $n-2 f$ then, appropriating the definition given in [6], we define a path of shape $\lambda$ in the Bratteli diagram associated with $B_{n}(q, r)$ to be a sequence of partitions

$$
\mathfrak{t}=\left(\lambda^{(0)}, \lambda^{(1)}, \ldots, \lambda^{(n)}\right)
$$

where $\lambda^{(0)}=\varnothing$ is the empty partition, $\lambda^{(n)}=\lambda$, and $\lambda^{(i-1)} \rightarrow \lambda^{(i)}$, whenever $1 \leq i \leq$ $n$. Let $\mathfrak{T}_{n}(\lambda)$ denote the set of paths of shape $\lambda$ in the Bratteli diagram of $B_{n}(q, r)$. If $\mathfrak{t}=\left(\lambda^{(0)}, \lambda^{(1)}, \ldots, \lambda^{(n)}\right)$ is in $\mathfrak{T}_{n}(\lambda)$, and $i$ is an integer, $0 \leq i \leq n$, define

$$
\left.\mathfrak{t}\right|_{i}=\left(\lambda^{(0)}, \lambda^{(1)}, \ldots, \lambda^{(i)}\right)
$$

The set $\mathfrak{T}_{n}(\lambda)$ is equipped with a dominance order $\unrhd$ defined as follows: given paths

$$
\mathfrak{t}=\left(\lambda^{(0)}, \lambda^{(1)}, \ldots, \lambda^{(n)}\right) \quad \text { and } \quad \mathfrak{u}=\left(\mu^{(0)}, \mu^{(1)}, \ldots, \mu^{(n)}\right)
$$

in $\mathfrak{T}_{n}(\lambda)$, write $\mathfrak{t} \unrhd \mathfrak{u}$ if $\lambda^{(k)} \unrhd \mu^{(k)}$ for $k=1,2, \ldots, n$. As usual, we write $\mathfrak{t} \triangleright \mathfrak{u}$ to mean that $\mathfrak{t} \unrhd \mathfrak{u}$ and $\mathfrak{t} \neq \mathfrak{u}$. There is a unique path in $\mathfrak{T}_{n}(\lambda)$ which is maximal with respect to the order $\unrhd$. Denote by $\mathfrak{t}^{\lambda}$ the maximal element in $\mathfrak{T}_{n}(\lambda)$.

Example 6.1 Let $n=10, f=2$ and $\lambda=(3,2,1)$. Then

$$
\mathfrak{t}^{\lambda}=(\varnothing, \square, \varnothing, \square, \varnothing, \square, \square, \square, \square, \square, \square, \square, \square, \square, \square, \square, \square)
$$

is the maximal element in $\mathfrak{T}_{n}(\lambda)$ with respect to the order $\unrhd$.

Let $\lambda$ be a partition of $n-2 f$, for $0 \leq f \leq[n / 2]$. Theorem 5.9 will now be applied iteratively to give the $B_{n}(q, r)$-module $S^{\lambda}$ a generic basis indexed by the set $\mathfrak{T}_{n}(\lambda)$.

Assume that for each partition $\mu$ with $\mu \rightarrow \lambda$, we have defined a set

$$
\left\{m_{\mathfrak{u}}=m_{\mu} b_{\mathfrak{u}}+\check{B}_{n-1}^{\mu}: \mathfrak{u} \in \mathfrak{T}_{n-1}(\mu)\right\}
$$


which freely generates $S^{\mu}$ as an $R$-module. To define $\left\{b_{\mathfrak{t}}: \mathfrak{t} \in \mathfrak{T}_{n}(\lambda)\right\}$, we refer to the definition of $y_{\mu}^{\lambda}$ given in (5.4) and (5.21), and write

$$
m_{\mathfrak{t}}=y_{\mu}^{\lambda} b_{\mathfrak{u}} \quad \text { whenever } \mathfrak{u} \in \mathfrak{T}_{n-1}(\mu) \text { and }\left.\mathfrak{t}\right|_{n-1}=\mathfrak{u} .
$$

By Theorem 3.1 there exist $a_{w}$, for $w \in \mathfrak{S}_{n}$, depending only on $b_{\mathfrak{u}}$, such that the term $y_{\mu}^{\lambda} b_{\mathfrak{u}}$ on the right hand side of the expression (6.2) can be expressed in terms of the basis (3.5) as

$$
m_{\mathfrak{t}}=y_{\mu}^{\lambda} b_{\mathfrak{u}}=\sum_{w \in \mathfrak{S}_{n}} a_{w} m_{\lambda} T_{w}+\check{B}_{n}^{\lambda} .
$$

Thus, given $\mathfrak{t} \in \mathfrak{T}_{n}(\lambda)$ and $\mathfrak{u} \in \mathfrak{T}_{n-1}(\mu)$ with $\left.\mathfrak{t}\right|_{n-1}=\mathfrak{u}$, define

$$
b_{\mathfrak{t}}=\sum_{w \in \mathfrak{S}_{n}} a_{w} T_{w}
$$

where the elements $a_{w} \in R$, for $w \in \mathfrak{S}_{n}$, are determined uniquely by the basis (3.5) and the expression (6.3).

From Theorem 5.9 it follows that set

$$
\left\{m_{\mathfrak{t}}=m_{\lambda} b_{\mathfrak{t}}+\check{B}_{n}^{\lambda}: \mathfrak{t} \in \mathfrak{T}_{n}(\lambda)\right\}
$$

constructed by the above procedure is a basis for $S^{\lambda}$ over $R$ and that, for $1 \leq i \leq n$, the basis (6.5) admits natural filtrations by $B_{i}(q, r)$-modules, which is analogous to the property of the Murphy basis for $\mathscr{H}_{n}\left(q^{2}\right)$ given in Lemma 2.3.

With little further ado, the above construction allows us to write the following.

Theorem 6.1 The algebra $B_{n}(q, r)$ is freely generated as an $R$ module by the collection

$$
\mathcal{M}=\left\{m_{\mathfrak{s t}}=b_{\mathfrak{s}}^{*} m_{\lambda} b_{\mathfrak{t}}: \mathfrak{s}, \mathfrak{t} \in \mathfrak{T}_{n}(\lambda), \lambda \vdash n-2 f, \text { and } 0 \leq f \leq[n / 2]\right\} .
$$

Moreover the following statements hold:

1. The algebra anti-involution $*$ satisfies $*: m_{\mathfrak{s t}} \mapsto m_{\mathfrak{t} \mathfrak{s}}$, for all $m_{\mathfrak{s t}} \in \mathcal{M}$;

2. Suppose that $b \in B_{n}(q, r)$ and let $f$ be an integer $0 \leq f \leq[n / 2]$. If $\lambda$ is a partition of $n-2 f$ and $\mathfrak{t} \in \mathfrak{T}_{n}(\lambda)$, then there exist $a_{\mathfrak{v}} \in R$, for $\mathfrak{v} \in \mathfrak{T}_{n}(\lambda)$, such that, for all $\mathfrak{s} \in \mathfrak{T}_{n}(\lambda)$,

$$
m_{\mathfrak{s t}} b \equiv \sum_{\mathfrak{v} \in \mathfrak{T}_{n}(\lambda)} a_{\mathfrak{v}} m_{\mathfrak{s v}} \quad \bmod \check{B}_{n}^{\lambda}
$$

If $\lambda$ is a partition of $n-2 f$, then as a consequence of the theorem, $\check{B}_{n}^{\lambda}$ is the free $R$-module generated by $\left\{m_{\mathfrak{s t}}: \mathfrak{s}, \mathfrak{t} \in \mathfrak{T}_{n}(\mu)\right.$ and $\left.\mu \triangleright \lambda\right\}$.

Example 6.2 We explicitly compute a basis of the form displayed in (6.5) for the $B_{4}(q, r)$-modules $S^{\lambda}$ and $S^{\lambda^{\prime}}$ where $\lambda=(2)$ and $\lambda^{\prime}=(1,1)$. Our iterative construction the basis for $S^{\lambda}$ entails explicit computation of $b_{\mathfrak{u}}$, for all $\mathfrak{u} \in \mathfrak{T}_{i}\left(\lambda^{(i)}\right)$ for which

$$
\left(\varnothing, \ldots, \lambda^{(i-1)}, \lambda^{(i)}, \ldots, \lambda\right) \in \mathfrak{T}_{4}(\lambda),
$$

with similar requirements for computing the basis for $S^{\lambda^{\prime}}$. 
(a) The algebra $B_{2}(q, r)$ has three one dimensional cell modules; if $\mu$ is one of the partitions $\varnothing,(2)$ or $(1,1)$, associate to the path in $\mathfrak{T}_{2}(\mu)$ an element of $S^{\mu}$ as

$$
\begin{aligned}
(\varnothing, \square, \varnothing) & \mapsto E_{1} ; \\
(\varnothing, \square, \square) & \mapsto\left(1+q T_{1}\right)+\check{B}_{2}^{(2)} \\
(\varnothing, \square, \square) & \mapsto 1+B_{2}^{(1,1)},
\end{aligned}
$$

to obtain a cellular basis for $B_{2}(q, r)$ which is compatible with the ordering of partitions $\varnothing \triangleright(2) \triangleright(1,1)$.

(b) The algebra $B_{3}(q, r)$ has four cell modules, one corresponding to each of the partitions, (1) $\triangleright(3) \triangleright(2,1) \triangleright\left(1^{3}\right)$.

(i) If $\mu=(1)$ then $\breve{B}_{3}^{\mu}=0$ and $m_{\mu}=E_{1}$; since $v \rightarrow \mu$ precisely if $v$ is one of $\varnothing \triangleright(2) \triangleright(1,1)$, using part $(a)$ above, we associate to each path in $\mathfrak{T}_{3}(\mu)$ an element of $S^{\mu}$ as

$$
\begin{aligned}
(\varnothing, \square, \varnothing, \square) & \mapsto \quad m_{\mathfrak{t}^{\mu}}=E_{1} ; \\
(\varnothing, \square, \square, \square) & \mapsto m_{\mathfrak{t}^{\mu}} T_{2}^{-1} T_{1}^{-1}\left(1+q T_{1}\right) ; \\
(\varnothing, \square, \square, \square) & \mapsto m_{\mathfrak{t}^{\mu}} T_{2}^{-1} T_{1}^{-1}=m_{\mathfrak{t}^{\mu}} T_{2} T_{1} .
\end{aligned}
$$

The transition matrix from the basis $\left\{m_{\mathfrak{t}}=m_{\lambda} b_{\mathfrak{t}}+\check{B}_{3}^{\mu}: \mathfrak{t} \in \mathfrak{T}_{3}(\mu)\right\}$ for $S^{\mu}$ given in (6.5) and ordered by dominance as above, to the ordered basis

$$
\left\{\mathbf{v}_{i}=m_{\mu} T_{v_{i}}: v_{1}=1, v_{2}=s_{2}, v_{3}=s_{2} s_{1}\right\}
$$

for $S^{\mu}$ given in (3.5) is:

$$
\left[\begin{array}{ccc}
1 & 1-q^{2} & 0 \\
0 & q & 0 \\
0 & q^{2} & 1
\end{array}\right]
$$

The elements $\left\{b_{\mathfrak{t}}: \mathfrak{t} \in \mathfrak{T}_{3}(\mu)\right\}$ of (6.5) are made explicit by the above transition matrix.

(ii) If $\mu=(3)$, then $S^{\mu}$ is one-dimensional and

$$
\left(\varnothing, \square, \square, \square, \square(\square) \mapsto m_{\mathfrak{t}^{\mu}}=\left(1+q T_{1}\right)\left(1+q T_{2}+q^{2} T_{2} T_{1}\right)+\check{B}_{3}^{(3)} .\right.
$$

(iii) If $\mu=(2,1)$, then $m_{\mu}=\left(1+q T_{1}\right)$ and a basis for $S^{\mu}$ is obtained by associating to each path in $\mathfrak{T}_{3}(\mu)$ an element as

$$
\begin{aligned}
(\varnothing, \square, \square, \square) & \mapsto m_{\mathfrak{t}^{\mu}}=\left(1+q T_{1}\right)+\check{B}_{3}^{(2,1)} ; \\
(\varnothing, \square, \square, \square) & \mapsto m_{\mathfrak{t}^{\mu}} T_{2} .
\end{aligned}
$$


(iv) Finally, if $\mu=(1,1,1)$, then $S^{\mu}$ is the right $B_{3}(q, r)$-module generated by $1+\check{B}_{3}^{(1,1,1)}$.

(c) Let $n=4$ and $\lambda=(2)$. Then $m_{\lambda}=E_{1}\left(1+q T_{3}\right)$ and $\mu \rightarrow \lambda$ if $\mu$ is one of the partitions $\mu^{(1)}=(1) \triangleright \mu^{(2)}=(3) \triangleright \mu^{(3)}=(2,1)$. Thus, based on $(b)$ above, we associate to each path $\mathfrak{t} \in \mathfrak{T}_{4}(\lambda)$ a basis element of the cell module $S^{\lambda}$ as follows:

$$
\begin{aligned}
& (\varnothing, \square, \varnothing, \square, \square) \mapsto y_{\mu^{(1)}}^{\lambda}=m_{\mathfrak{t}^{\lambda}}=E_{1}\left(1+q T_{3}\right)+\check{B}_{4}^{\lambda} ; \\
& (\varnothing, \square, \square, \square, \square) \mapsto y_{\mu^{(1)}}^{\lambda} T_{2}^{-1} T_{1}^{-1}\left(1+q T_{1}\right) \text {; } \\
& \varnothing, \square, \square, \square, \square(\square) \mapsto y_{\mu^{(1)}}^{\lambda} T_{2}^{-1} T_{1}^{-1}=m_{\mathfrak{t}^{\lambda}} T_{2} T_{1} ; \\
& (\varnothing, \square, \square, \square, \square \square, \square \square) \mapsto y_{\mu^{(2)}}^{\lambda}=E_{1}\left(T_{2} T_{1} T_{3} T_{2}\right)^{-1} m_{\mu^{(2)}}+\check{B}_{4}^{\lambda} \\
& =E_{1}\left(1+q T_{3}\right)\left(T_{2} T_{1} T_{3} T_{2}\right)^{-1} \\
& \times\left(1+q T_{2}+q^{2} T_{2} T_{1}\right)+\check{B}_{4}^{\lambda} \\
& =m_{\mathfrak{t}^{\lambda}}\left(T_{2} T_{1} T_{3} T_{2}\right)^{-1}\left(1+q T_{2}+q^{2} T_{2} T_{1}\right) \text {; } \\
& \left(\varnothing, \square, \square, \square, \square, \square(\square) \mapsto y_{\mu^{(3)}}^{\lambda}=E_{1}\left(T_{2} T_{1} T_{3} T_{2}\right)^{-1} m_{\mu^{(3)}}+\check{B}_{4}^{\lambda}\right. \\
& =m_{\mathfrak{t}^{\lambda}}\left(T_{2} T_{1} T_{3} T_{2}\right)^{-1}=m_{\mathfrak{t}^{\lambda}} T_{2} T_{3} T_{1} T_{2} ; \\
& (\varnothing, \square, \square, \square, \square, \square) \mapsto y_{\mu^{(3)}}^{\lambda} T_{2}=m_{\mathfrak{t}^{\lambda}}\left(T_{2} T_{1} T_{3} T_{2}\right)^{-1} T_{2} \\
& =m_{\mathfrak{t}^{\lambda}} T_{2}^{-1} T_{3}^{-1} T_{1}^{-1} .
\end{aligned}
$$

Expanding the terms on the right hand side above using results from Sect. 3 of [4], we obtain the transition matrix from the basis $\left\{m_{\mathfrak{t}}=m_{\lambda} b_{\mathfrak{t}}+\check{B}_{4}^{\lambda}: \mathfrak{t} \in \mathfrak{T}_{4}(\lambda)\right\}$ for $S^{\lambda}$ given in (6.5) and ordered by dominance as above, to basis

$$
\left\{\mathbf{v}_{i, j}=m_{\lambda} T_{v_{i, j}}+\check{B}_{4}^{\lambda}: v_{i, j}=s_{2} s_{3} \cdots s_{j-1} s_{1} s_{2} \cdots s_{i-1}\right\}
$$

for $S^{\lambda}$ given in (3.5), ordered lexicographically, as:

$$
\left[\begin{array}{cccccc}
1 & 1-q^{2} & 0 & 1-q^{2} & 0 & 0 \\
0 & q & 0 & q\left(1-q^{2}\right) & 0 & 0 \\
0 & 0 & 0 & q^{2} & 0 & 0 \\
0 & q^{2} & 1 q^{2}\left(1-q^{2}\right) & 0 & \frac{1-q^{2}}{q} \\
0 & 0 & 0 & q^{3} & 0 & 1 \\
0 & 0 & 0 & q^{4} & 1 \frac{q^{2}-1}{q}
\end{array}\right]
$$

It may be observed that the elements $\left\{b_{\mathfrak{t}}: \mathfrak{t} \in \mathfrak{T}_{4}(\lambda)\right\}$, given by the above matrix, are consistent with (6.6) above and reflect the existence of an embedding $S^{\mu^{(1)}} \hookrightarrow S^{\lambda}$ of $B_{3}(q, r)$-modules, as $N^{\mu^{(1)}} / \check{N}^{\mu^{(1)}} \cong S^{\mu^{(1)}}$, where $\check{N}^{\mu^{(1)}}=0$.

(d) Now consider the partition $\lambda^{\prime}=(1,1)$; here $m_{\lambda^{\prime}}=E_{1}$ and $\mu \rightarrow \lambda^{\prime}$ if $\mu$ is one of the partitions $\mu^{(1)}=(1) \triangleright \mu^{(2)}=(2,1) \triangleright \mu^{(3)}=(1,1,1)$; thus, based on 
Example 5.3 and the calculations $(b)$ above, we associate to each path $\mathfrak{t} \in \mathfrak{T}_{4}\left(\lambda^{\prime}\right)$ a basis element in the cell module $S^{\lambda^{\prime}}$ as follows:

$$
\begin{aligned}
& (\varnothing, \square, \varnothing, \square, \square) \mapsto y_{\mu^{(1)}}^{\lambda^{\prime}}=m_{\mathfrak{t}^{\lambda^{\prime}}}=E_{1}+\check{B}_{4}^{\lambda^{\prime}} ; \\
& (\varnothing, \square, \square, \square, \square) \mapsto y_{\mu^{(1)}}^{\lambda^{\prime}} T_{2}^{-1} T_{1}^{-1}\left(1+q T_{1}\right) \text {; } \\
& \left(\varnothing, \square, \square, \square, \square \mapsto y_{\mu^{(1)}}^{\lambda^{\prime}} T_{2}^{-1} T_{1}^{-1}=m_{\mathfrak{t}^{\lambda}} T_{2} T_{1} ;\right. \\
& (\varnothing, \square, \square, \square, \square, \square) \mapsto y_{\mu^{(2)}}^{\lambda^{\prime}}=E_{1}\left(T_{2} T_{1} T_{3} T_{2}\right)^{-1} T_{2} m_{\mu^{(1)}}+\check{B}_{4}^{\lambda^{\prime}} \\
& =m_{\mathfrak{t}^{\prime}} T_{2}^{-1} T_{3}^{-1} T_{1}^{-1}\left(1+q T_{1}\right) \\
& (\varnothing, \square, \square, \square, \square) \mapsto y_{\mu^{(2)}}^{\lambda^{\prime}} T_{2}=m_{\mathfrak{t}^{\lambda^{\prime}}} T_{2}^{-1} T_{3}^{-1} T_{1}^{-1}\left(1+q T_{1}\right) T_{2} \text {; } \\
& (\varnothing, \square, \square, \square, \square) \mapsto y_{\mu^{(3)}}^{\lambda^{\prime}}=E_{1}\left(T_{2} T_{1} T_{3} T_{2}\right)^{-1} m_{\mu^{(2)}}+\check{B}_{4}^{\lambda^{\prime}} \\
& =m_{\mathfrak{t}^{\lambda^{\prime}}}\left(T_{2} T_{1} T_{3} T_{2}\right)^{-1}=m_{\mathfrak{t}^{\lambda^{\prime}}} T_{2} T_{3} T_{1} T_{2} .
\end{aligned}
$$

The transition matrix from the basis $\left\{m_{\mathfrak{t}}=m_{\lambda^{\prime}} b_{\mathfrak{t}}+\check{B}_{4}^{\lambda^{\prime}}: \mathfrak{t} \in \mathfrak{T}_{4}\left(\lambda^{\prime}\right)\right\}$ for $S^{\lambda^{\prime}}$ given in (6.5) and ordered by dominance, to the basis

$$
\left\{\mathbf{v}_{i, j}=m_{\lambda^{\prime}} T_{v_{i, j}}+\check{B}_{4}^{\lambda^{\prime}}: v_{i, j}=s_{2} s_{3} \cdots s_{j-1} s_{1} s_{2} \cdots s_{i-1}\right\}
$$

for $S^{\lambda^{\prime}}$ given in (3.5) and ordered lexicographically, is:

$$
\left[\begin{array}{cccccc}
1 & 1-q^{2} & 0 & q\left(q^{2}-1\right) & 1-q^{2} & 0 \\
0 & q^{2} & 0 & 1-q^{2} & \frac{q^{2}-1}{q} & 0 \\
0 & 0 & 0 & q & -1 & 0 \\
0 & q^{3} & 1 q\left(1-q^{2}\right) & \frac{1-q^{2}}{q r} & 0 \\
0 & 0 & 0 & q^{2} & 0 & 0 \\
0 & 0 & 0 & 0 & q^{2} & 1
\end{array}\right]
$$

The elements $\left\{b_{\mathfrak{t}}: \mathfrak{t} \in \mathfrak{T}_{n}\left(\lambda^{\prime}\right)\right\}$ are made explicit by the above transition matrix.

Example 6.3 Let $n=5$ and $\lambda=(2,1)$. Then $\mu \rightarrow \lambda$ if $\mu$ is one of the partitions

$$
\mu^{(1)}=(2) \triangleright \mu^{(2)}=(1,1) \triangleright \mu^{(3)}=(3,1) \triangleright \mu^{(4)}=(2,2) \triangleright \mu^{(5)}=(2,1,1) .
$$

By considering a suitable basis for $N^{\mu^{(2)}} / N^{\mu^{(1)}}$, we make explicit the elements $b_{\mathfrak{t}}$, for $\mathfrak{t} \in \mathfrak{T}_{n}(\lambda)$, defined by (6.3) and

$$
\left\{y_{\mu^{(2)}}^{\lambda} b_{\mathfrak{u}}=m_{\mathfrak{t}^{\lambda}} b_{\mathfrak{t}}: \mathfrak{t} \in \mathfrak{T}_{n}(\lambda) \text { and }\left.\mathfrak{t}\right|_{n-1}=\mathfrak{u} \in \mathfrak{T}_{n-1}\left(\mu^{(2)}\right)\right\} .
$$




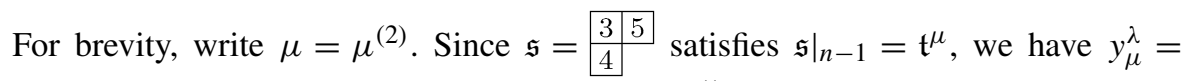
$m_{\mathfrak{t}^{\lambda}} T_{d(\mathfrak{s})}=m_{\mathfrak{t}^{\lambda}} T_{4}$, where $m_{\mathfrak{t}^{\lambda}}=E_{1}\left(1+q T_{3}\right)+\check{B}_{n}^{\lambda}$. The transition matrix from the basis

$$
\left\{y_{\mu}^{\lambda} b_{\mathfrak{u}}+\check{N}^{\mu}=m_{\mathfrak{t}^{\lambda}} b_{\mathfrak{t}}+\check{N}^{\mu}: \mathfrak{t} \in \mathfrak{T}_{n}(\lambda) \text { and }\left.\mathfrak{t}\right|_{n-1}=\mathfrak{u} \in \mathfrak{T}_{n-1}(\mu)\right\}
$$

which is ordered by dominance, to the basis

$$
\left\{\mathbf{v}_{i, j}=m_{\mathfrak{t}^{\lambda}} T_{d(\mathfrak{s})} T_{v_{i, j}}+\check{N}^{\mu}: v_{i, j}=s_{2} s_{3} \cdots s_{j-1} s_{1} s_{2} \cdots s_{i-1}\right\}
$$

which we order lexicographically, is given by (6.7) above. Observe that though $N^{\mu} / \check{N}^{\mu} \cong S^{\mu^{(2)}}$ as $B_{n-1}(q, r)$-modules, the construction does not give an embed$\operatorname{ding} S^{\mu^{(2)}} \hookrightarrow S^{\lambda}$ of $B_{n-1}(q, r)$-modules.

\section{Jucys-Murphy operators}

Define the operators $L_{i} \in B_{n}(q, r)$, for $i=1,2, \ldots, n$, by $L_{1}=1$ and $L_{i}=$ $T_{i-1} L_{i-1} T_{i-1}$ when $i=2, \ldots, n$. Let $\mathcal{L}=\mathcal{L}_{n}$ denote the subalgebra of $B_{n}(q, r)$ generated by $L_{1}, \ldots, L_{n}$. The next statement, which is the analogue to Proposition 2.4, is easily obtained from the braid relation $T_{i} T_{i+1} T_{i}=T_{i+1} T_{i} T_{i+1}$.

Proposition 7.1 Let $i$ and $k$ be integers, $1 \leq i<n$ and $1 \leq k \leq n$. Then the following statements hold.

1. $T_{i}$ and $L_{k}$ commute if $i \neq k-1, k$.

2. $L_{i}$ and $L_{k}$ commute.

3. $T_{i}$ commutes with $L_{i} L_{i+1}$.

4. $L_{2} \cdots L_{n}$ belongs to the centre of $B_{n}(q, r)$.

Remark 7.1 (i) The elements $L_{i}$ are a special case of certain operators defined in Corollary 1.6 of [6] in a context of semisimple path algebras.

(ii) The elements $L_{i}$ bear an analogy with the Jucys-Murphy operators $D_{i}$ defined in Sect. 2.2; we therefore refer to the $L_{i}$ as "Jucys-Murphy operators" in $B_{n}(q, r)$.

For integers $j, k$, with $1 \leq j, k \leq n$, define the elements $L_{k}^{(j)}$ by $L_{1}^{(j)}=1$ and

$$
L_{k}^{(j)}=T_{j+k-2} L_{k-1}^{(j)} T_{j+k-2}, \quad \text { for } k \geq 2 .
$$

In particular $L_{k}^{(1)}$, for $k=1, \ldots, n$, are the usual Jucys-Murphy operators in $B_{n}(q, r)$.

The next proposition is a step on the way to showing that the set $\left\{m_{\mathfrak{t}}=m_{\lambda} b_{\mathfrak{t}}+\check{B}_{n}^{\lambda}\right.$ : $\left.\mathfrak{t} \in \mathfrak{T}_{n}(\lambda)\right\}$ defined in (6.5) above is a basis of generalised eigenvectors for the action of Jucys-Murphy operators on the cell module $S^{\lambda}$. 
Proposition 7.2 Let $i, k$ be integers with $1 \leq i \leq n$ and $1<k \leq n$. Then

$$
E_{i} L_{k}^{(i)}= \begin{cases}r^{-2} E_{i} & \text { if } k=2 \\ E_{i} & \text { if } k=3 \\ E_{i} L_{k-2}^{(i+2)} & \text { if } k \geq 4\end{cases}
$$

Proof If $k=2$, then $E_{i} L_{k}^{(i)}=E_{i} T_{i}^{2}=r^{-2} E_{i}$. For $k=3$, we use the relations $E_{i} E_{i+1}=E_{i} T_{i+1} T_{i}=T_{i+1} T_{i} E_{i+1}$ and $E_{i} E_{i+1} E_{i}=E_{i}$ to obtain

$$
E_{i} L_{3}^{(i)}=E_{i} T_{i+1} T_{i} T_{i} T_{i+1}=E_{i} E_{i+1} T_{i} T_{i+1}=E_{i} E_{i+1} E_{i}=E_{i} .
$$

If $k \geq 4$, then using (7.1),

$$
\begin{aligned}
E_{i} L_{k}^{(i)} & =E_{i} T_{i+k-2} T_{i+k-3} \cdots T_{i+2} L_{3}^{(i)} T_{i+2} \cdots T_{i+k-3} T_{i+k-2} \\
& =T_{i+k-2} T_{i+k-3} \cdots T_{i+2} E_{i} L_{3}^{(i)} T_{i+2} \cdots T_{i+k-3} T_{i+k-2} \\
& =E_{i} T_{i+k-2} T_{i+k-3} \cdots T_{i+2} T_{i+2} \cdots T_{i+k-3} T_{i+k-2}=E_{i} L_{k-2}^{(i+2)}
\end{aligned}
$$

Corollary 7.3 Let $f, k$ be integers, $0<f \leq[n / 2]$ and $1 \leq k \leq n$. Then

$$
E_{1} E_{3} \cdots E_{2 f-1} L_{k}= \begin{cases}E_{1} E_{3} \cdots E_{2 f-1} & \text { if } k \text { is odd, } 1 \leq k \leq 2 f+1 \\ r^{-2} E_{1} E_{3} \cdots E_{2 f-1} & \text { if } k \text { is even, } 1<k \leq 2 f \\ E_{1} E_{3} \cdots E_{2 f-1} L_{k-2 f}^{(2 f+1)} & \text { if } 2 f+1<k \leq n\end{cases}
$$

Proof If $k$ is odd, $1<k \leq 2 f+1$, then by the proposition above,

$$
\begin{gathered}
E_{1} E_{3} \cdots E_{k} L_{k}=E_{1} E_{3} \cdots E_{k} L_{k}^{(1)}=E_{1} E_{3} \cdots E_{k} L_{k-2}^{(3)}=\cdots \\
\cdots=E_{1} E_{3} \cdots E_{k} L_{1}^{(k)}=E_{1} E_{3} \cdots E_{k}
\end{gathered}
$$

Since $E_{k+2} E_{k+4} \cdots E_{2 f-1}$ commutes with $L_{k}$, the first statement has been proved. If $k$ is even, $1<k \leq 2 f$, then use the relation $E_{i} T_{i}=r^{-1} T_{i}$ and (7.2) so that

$$
\begin{aligned}
E_{1} E_{3} \cdots E_{2 f-1} L_{k} & =E_{1} E_{3} \cdots E_{2 f-1} T_{k-1} L_{k-1} T_{k-1} \\
& =r^{-1} E_{1} E_{3} \cdots E_{2 f-1} L_{k-1} T_{k-1}=r^{-2} E_{1} E_{3} \cdots E_{2 f-1},
\end{aligned}
$$

as above. The final case where $2 f+1<k \leq n$ is similar to (7.2) above.

Let $f$ be an integer, $0 \leq f \leq[n / 2]$, and $\lambda$ be a partition of $n-2 f$. Suppose that $\mathfrak{t}=\left(\lambda^{(0)}, \lambda^{(1)}, \ldots, \lambda^{(n)}\right)$ is a path in $\mathfrak{T}_{n}(\lambda)$, and that $k$ is an integer, $1 \leq k \leq n$. Then generalise the definition (2.10) by writing

$$
P_{\mathfrak{t}}(k)= \begin{cases}q^{2(j-i)} & \text { if }\left[\lambda^{(k)}\right]=\left[\lambda^{(k-1)}\right] \cup\{(i, j)\} \\ q^{2(i-j)} r^{-2} & \text { if }\left[\lambda^{(k)}\right]=\left[\lambda^{(k-1)}\right] \backslash\{(i, j)\} .\end{cases}
$$


Since $q$ does not have finite multiplicative order in $R$, the next result which is similar in flavour to Lemma 5.20 of [6], follows immediately from Lemma 3.34 of [8].

Lemma 7.4 Let $f$ be an integer $0 \leq f<[n / 2]$ and $\lambda^{(n-1)}$ be a partition of $n-1-$ $2 f$. If $\mathfrak{s}=\left(\lambda^{(0)}, \lambda^{(1)}, \ldots, \lambda^{(n-1)}\right)$ is a path in $\mathfrak{T}_{n-1}\left(\lambda^{(n-1)}\right)$, then the terms $\left(P_{\mathfrak{t}}(n)\right.$ : $\left.\left.\mathfrak{t}\right|_{n-1}=\mathfrak{s}\right)$ are all distinct.

The next proposition is essentially a restatement of Theorem 2.5. Recall that if $f$ is an integer, $0 \leq f \leq[n / 2]$, and $\lambda$ is a partition of $n-2 f$, then $\mathfrak{t}^{\lambda}$ is the element in $\mathfrak{T}_{n}(\lambda)$ which is maximal under the dominance order.

Proposition 7.5 If $\lambda$ is a partition of $n$ and $k$ is an integer $1 \leq k \leq n$, then $m_{\mathfrak{t}^{\lambda}} L_{k}=$ $P_{\mathfrak{t}^{\lambda}}(k) m_{\mathfrak{t}^{\lambda}}$.

Proof By definition, $m_{\mathfrak{t}^{\lambda}}=m_{\lambda}+\check{B}_{n}^{\lambda}$ so, using the property (3.8),

$$
m_{\lambda} L_{k}+B_{n}^{1}=\vartheta_{0}\left(c_{\lambda} D_{k}\right)=P_{\mathfrak{t}^{\lambda}}(k) \vartheta_{0}\left(c_{\lambda}\right)
$$

where the last equality follows from Theorem 2.5. Now, given that $B_{n}^{1} \subseteq \check{B}_{n}^{\lambda}$ whenever $\lambda$ is a partition of $n$, the result follows.

Proposition 7.6 Let $f$ be an integer, $0<f \leq[n / 2]$, and $\lambda$ be a partition of $n-2 f$. Then $m_{\mathfrak{t}^{\lambda}} L_{k}=P_{\mathfrak{t}^{\lambda}}(k) m_{\mathfrak{t}^{\lambda}}$.

Proof If $k$ is an integer, $1 \leq k \leq 2 f+1$, the statement follows from Corollary 7.3; otherwise, using the corollary and property (3.8),

$$
\begin{aligned}
m_{\lambda} L_{k}+B_{n}^{f+1} & =x_{\lambda} E_{1} E_{3} \cdots E_{2 f-1} L_{k}+B_{n}^{f+1} \\
& =x_{\lambda} E_{1} E_{3} \cdots E_{2 f-1} L_{k-2 f}^{(2 f+1)}+B_{n}^{f+1} \\
& =\vartheta_{f}\left(c_{\lambda} D_{k-2 f}\right)=P_{\hat{\mathfrak{t}}^{\lambda}}(k-2 f) \vartheta_{f}\left(c_{\lambda}\right) \\
& =P_{\mathfrak{t}^{\lambda}}(k) m_{\lambda}+B_{n}^{f+1},
\end{aligned}
$$

whence the result follows, since $B_{n}^{f+1} \subseteq \check{B}_{n}^{\lambda}$ whenever $\lambda$ is a partition of $n-2 f$.

Proposition 7.7 Let $f$ be an integer, $0 \leq f \leq[n / 2]$ and $\lambda$ be a partition of $n-2 f$. Then there exists an invariant $\alpha \in R$ such that $\left(L_{2} \cdots L_{n}\right)$ acts on $S^{\lambda}$ as a multiple by $\alpha$ of the identity.

Proof Consider an element $\sum_{w \in \mathfrak{S}_{n}} a_{w} m_{\lambda} T_{w}+\check{B}_{n}^{\lambda}$, for $a_{w} \in R$. Since $\left(L_{2} \cdots L_{n}\right)$ is central in $B_{n}(q, r)$,

$$
\sum_{w \in \mathfrak{S}_{n}} a_{w} m_{\lambda} T_{w}\left(L_{2} \cdots L_{n}\right)=\sum_{w \in \mathfrak{S}_{n}} a_{w} m_{\lambda}\left(L_{2} \cdots L_{n}\right) T_{w},
$$

so $\alpha=\prod_{k=2}^{n} P_{\mathfrak{t}^{\lambda}}(k)$, by the previous proposition. 
For the proof of Theorem 7.8 we use the filtration of the $B_{n}(q, r)$ module $S^{\lambda}$ by $B_{n-1}(q, r)$-modules given in Theorem 5.9.

Theorem 7.8 Let $f$ be an integer, $0 \leq f \leq[n / 2]$, and $\lambda$ be a partition of $n-2 f$. If $\mathfrak{t} \in \mathfrak{T}_{n}(\lambda)$, then there exist $a_{\mathfrak{u}} \in R$, for $\mathfrak{u} \in \mathfrak{T}_{n}(\lambda)$, such that

$$
m_{\mathfrak{t}} L_{k}=P_{\mathfrak{t}}(k) m_{\mathfrak{t}}+\sum_{\substack{\mathfrak{u} \in \mathfrak{T}_{n}(\lambda) \\ \mathfrak{u} \triangleright \mathfrak{t}}} a_{\mathfrak{u}} m_{\mathfrak{u}} .
$$

Proof We proceed by induction. Let $\mathfrak{t}$ be in $\mathfrak{T}_{n}(\lambda)$ and suppose that $\mathfrak{s}=\left.\mathfrak{t}\right|_{n-1}$ is an element of $\mathfrak{T}_{n-1}(\mu)$. Then $m_{\mathfrak{t}}+\check{N}^{\mu} \mapsto m_{\mathfrak{s}}$ under the isomorphism $N^{\mu} / \check{N}^{\mu} \rightarrow S^{\mu}$ of $B_{n-1}(q, r)-$ modules given in Theorem 5.9. Hence, if $1 \leq k<n$, there exist $a_{\mathfrak{v}} \in R$, for $\mathfrak{v} \in \mathfrak{T}_{n-1}(\mu)$, such that

$$
m_{\mathfrak{t}} L_{k}+\check{N}^{\mu} \mapsto P_{\mathfrak{s}}(k) m_{\mathfrak{s}}+\sum_{\substack{\mathfrak{v} \in \mathfrak{T}_{n-1}(\mu) \\ \mathfrak{v} \triangleright \mathfrak{s}}} a_{\mathfrak{v}} m_{\mathfrak{v}}
$$

under the $B_{n-1}(q, r)$-module isomorphism $N^{\mu} / \check{N}^{\mu} \rightarrow S^{\mu}$. Thus the $a_{\mathfrak{v}} \in R$, for $\mathfrak{v} \in \mathfrak{T}_{n-1}(\mu)$, satisfy

$$
m_{\mathfrak{t}} L_{k} \equiv P_{\mathfrak{s}}(k) m_{\mathfrak{t}}+\sum_{\substack{\mathfrak{v} \in \mathfrak{T}_{n-1}(\mu) \\ \mathfrak{v} \triangleright \mathfrak{s}}} a_{\mathfrak{v}} y_{\mu}^{\lambda} b_{\mathfrak{v}} \quad \bmod \check{N}^{\mu}
$$

If $\mathfrak{v} \in \mathfrak{T}_{n-1}(\mu)$ and $\mathfrak{v} \triangleright \mathfrak{s}$, then, using the definition (6.3), $y_{\mu}^{\lambda} b_{\mathfrak{v}}=m_{\mathfrak{u}}$, where $\left.\mathfrak{u}\right|_{n-1}=$ $\mathfrak{v} \triangleright \mathfrak{s}=\left.\mathfrak{t}\right|_{n-1}$, and thus $\mathfrak{u} \triangleright \mathfrak{t}$. Since $P_{\mathfrak{t}}(k)=P_{\mathfrak{s}}(k)$ whenever $1 \leq k<n$, the above expression becomes

$$
m_{\mathfrak{t}} L_{k} \equiv P_{\mathfrak{t}}(k) m_{\mathfrak{t}}+\sum_{\substack{\mathfrak{u} \in \mathfrak{T}_{n}(\lambda) \\ \mathfrak{u} \triangleright \mathfrak{t}}} a_{\mathfrak{u}} m_{\mathfrak{u}} \bmod \check{N}^{\mu}
$$

where $a_{\mathfrak{u}}=a_{\mathfrak{v}}$ whenever $\left.\mathfrak{u}\right|_{n-1}=\mathfrak{v}$. Now, $\check{N}^{\mu}$ is the $B_{n-1}(q, r)$-module freely generated by

$$
\left\{m_{\mathfrak{u}}=y_{v}^{\lambda} b_{\mathfrak{v}}: \mathfrak{u} \in \mathfrak{T}_{n}(\lambda), v \rightarrow \lambda, \nu \triangleright \mu \text { and }\left.\mathfrak{u}\right|_{n-1}=\mathfrak{v} \in \mathfrak{T}_{n-1}(v)\right\}
$$

and so it follows that $\check{N}^{\mu}$ is contained in the $R$-submodule of $S^{\lambda}$ generated by $\left\{m_{\mathfrak{u}}\right.$ : $\mathfrak{u} \in \mathfrak{T}_{n}(\lambda)$ and $\left.\mathfrak{u} \triangleright \mathfrak{t}\right\}$. Thus (7.3) shows that the theorem holds true whenever $1 \leq k<$ $n$.

That $L_{n}$ acts triangularly on $S^{\lambda}$, can now be deduced using Proposition 7.7:

$$
m_{\mathfrak{t}} L_{n}=\prod_{k=1}^{n} P_{\mathfrak{t}}(k) m_{\mathfrak{t}}\left(L_{2} L_{3} \cdots L_{n-1}\right)^{-1} .
$$

Thus the generalised eigenvalue for $L_{n}$ acting on $m_{\mathfrak{t}}$ is $P_{\mathfrak{t}}(n)$. 


\section{Semisimplicity criteria for B-M-W algebras}

Let $\kappa$ be a field and take $\hat{q}, \hat{r},\left(\hat{q}-\hat{q}^{-1}\right)$ to be units in $\kappa$. In this section we consider the algebra $B_{n}(\hat{q}, \hat{r})=B_{n}(q, r) \otimes_{R} \kappa$. For $\mathfrak{t} \in \mathfrak{T}_{n}(\lambda)$ and $k=1, \ldots, n$, let $\hat{P}_{\mathfrak{t}}(k)$ denote the evaluation of the monomial $P_{\mathfrak{t}}(k)$ at $(\hat{q}, \hat{r})$,

$$
\hat{P}_{\mathfrak{t}}(k)= \begin{cases}\hat{q}^{2(j-i)} & \text { if }\left[\lambda^{(k)}\right]=\left[\lambda^{(k-1)}\right] \cup\{(i, j)\} \\ \hat{q}^{2(i-j)} \hat{r}^{-2} & \text { if }\left[\lambda^{(k)}\right]=\left[\lambda^{(k-1)}\right] \backslash\{(i, j)\},\end{cases}
$$

and define the ordered $n$-tuple $\hat{P}(\mathfrak{t})=\left(\hat{P}_{\mathfrak{t}}(1), \ldots, \hat{P}_{\mathfrak{t}}(n)\right)$. The next statement is the counterpart to Proposition 3.37 of [8].

Proposition 8.1 Let $f$ be an integer, $0 \leq f \leq[n / 2]$, and $\lambda$ be a partition of $n-2 f$.

(i) Let $\rho=\left(\rho_{1}, \ldots, \rho_{n}\right)$ be a sequence of elements of $\kappa$ such that there exists a path $\mathfrak{t} \in \mathfrak{T}_{n}(\lambda)$ with $\rho=\hat{P}(\mathfrak{t})$. Then there exists a one-dimensional $\mathcal{L}$-module $\mathcal{L}_{\rho}=\kappa x_{\rho}$ such that

$$
x_{\rho} L_{k}=\rho_{k} x_{\rho} \quad \text { for } k=1,2, \ldots, n .
$$

Moreover, every irreducible $\mathcal{L}$-module has this form.

(ii) Let $f$ be an integer, $0 \leq f \leq[n / 2]$, and suppose that $\lambda$ is a partition of $n-2 f$. Fix an ordering $\mathfrak{t}_{1}, \ldots, \mathfrak{t}_{k}=\mathfrak{t}^{\lambda}$ of $\mathfrak{T}_{n}(\lambda)$ so that $i>j$ whenever $\mathfrak{t}_{i} \triangleright \mathfrak{t}_{j}$. Then $S^{\lambda}$ has a $\mathcal{L}$-module composition series

$$
S^{\lambda}=S_{1}>S_{2}>\cdots>S_{k}>S_{k+1}=0
$$

such that $S_{i} / S_{i+1}=\mathcal{L}_{\rho^{i}}$, for each $i$, where $\rho^{i}=\hat{P}\left(\mathfrak{t}_{i}\right)$.

Proof As in [8], we prove (ii) from which item (i) will follow. Order the elements of $\mathfrak{T}_{n}(\lambda)$ as in item (ii), and for $i=1, \ldots, k$, let $S_{i}$ be the $\kappa$-module generated by $\left\{m_{\mathfrak{t}_{j}}: i \leq j \leq k\right\}$. By Theorem 7.8, each $S_{i}$ is an $\mathcal{L}$-module, and so $S^{\lambda}=S_{1}>\cdots>$ $S_{k}>0$ is an $\mathcal{L}$-module filtration of $S^{\lambda}$. Further, by Theorem 7.8 again, $S_{i} / S_{i+1}=$ $\kappa\left(m_{\mathfrak{t}_{i}}+S_{i+1}\right)$ is a one dimensional module isomorphic to $\mathcal{L}_{i}$.

Theorem 8.2 Suppose that for each pair of partitions $\lambda$ of $n-2 f$ and $\mu$ of $n-2 f^{\prime}$, for integers $f, f^{\prime}$ with $0 \leq f, f^{\prime} \leq[n / 2]$, and that for each pair of paths $\mathfrak{s} \in \mathfrak{T}_{n}(\lambda)$ and $\mathfrak{t} \in \mathfrak{T}_{n}(\mu)$, the conditions $\lambda \unrhd \mu$ and $\hat{P}(\mathfrak{s})=\hat{P}(\mathfrak{t})$ together imply that $\lambda=\mu$. Then $B_{n}(\hat{q}, \hat{r})$ is a semisimple algebra over $\kappa$.

Proof The hypotheses of the theorem imply that given a pair of partitions $\lambda$ and $\mu$ with $\lambda \triangleright \mu$, there are no $\mathcal{L}$-module composition factors in common between $S^{\lambda}$ and $S^{\mu}$. However, if $B_{n}(\hat{q}, \hat{r})$ is not semisimple, then using Theorem $4.4, D^{\mu}$ is a $B_{n}(\hat{q}, \hat{r})$-module composition factor of $S^{\lambda}$ for some pair of partitions $\lambda$ and $\mu$ for which, by Proposition 3.6 of [5], $\lambda \triangleright \mu$; in particular, by Proposition 8.1 , there must be $\mathcal{L}$-module composition factors in common between $S^{\lambda}$ and $S^{\mu}$, which as already noted, is an impossibility. 
From the next statement (Lemma 5.20 of [6]), it will follow that the Jucys-Murphy operators do in fact distinguish between cell modules of $B_{n}(q, r)$.

Lemma 8.3 Let $f$ be an integer $0 \leq f<[n / 2]$ and $\lambda^{(n-1)}$ be a partition of $n-1-$ $2 f$. If $\mathfrak{s}=\left(\lambda^{(0)}, \lambda^{(1)}, \ldots, \lambda^{(n-1)}\right)$ is a path in $\mathfrak{T}_{n-1}\left(\lambda^{(n-1)}\right)$, then the terms $\left(P_{\mathfrak{t}}(n)\right.$ : $\left.\left.\mathfrak{t}\right|_{n-1}=\mathfrak{s}\right)$ are all distinct .

For the case where $\kappa=\mathbb{C}(\hat{q}, \hat{r})$, a form of the following statement can be found in Corollary 5.6 of [12].

Corollary 8.4 If $\kappa$ is a field, then a $B-M-W$ algebra $B_{n}(\hat{q}, \hat{r})$ over $\kappa$ is semisimple for almost all (all but finitely many) choices of the parameters $\hat{q}$ and $\hat{r}$. If $B_{n}(\hat{q}, \hat{r})$ is not semisimple then necessarily $\hat{q}$ is a root of unity or $\hat{r}= \pm \hat{q}^{k}$ for some integer $k$.

Theorem 8.5 may be compared with Theorem 11.2 below. Theorem 8.5 gives a semisimplicity criterion for $B_{n}(q, r)$.

Theorem 8.5 Let $\lambda$ be a partition of $n-2 f$ and $\mu$ be a partition of $n-2 g$, where $0 \leq f<g \leq[n / 2]$. If $\operatorname{Hom}_{B_{n}(\hat{q}, \hat{r})}\left(S^{\lambda}, S^{\mu}\right) \neq 0$, then

$$
\hat{r}^{2(g-f)} \hat{q}^{2 \sum_{(i, j) \in[\lambda]}(j-i)}=\hat{q}^{2 \sum_{(i, j) \in[\mu]}(j-i)} .
$$

Proof Suppose that $\mathbf{u} \in S^{\lambda}, \mathbf{v} \in S^{\mu}$ are non-zero and that $\mathbf{u} \mapsto \mathbf{v}$ under some element in $\operatorname{Hom}_{B_{n}(\hat{q}, \hat{r})}\left(S^{\lambda}, S^{\mu}\right)$. Then, using Lemma 7.7, on the one hand $\mathbf{u}\left(L_{2} L_{3} \cdots L_{n}\right)=$ $\hat{r}^{-2 f} \hat{q}^{2 \sum_{(i, j) \in[\lambda]}(j-i)} \mathbf{u}$, while on the other $\mathbf{v} L_{2} L_{3} \cdots L_{n}=\hat{r}^{-2 g} \hat{q}^{2 \sum_{(i, j) \in[\mu]}(j-i)} \mathbf{v}$. Since $\mathbf{v}$ is the homomorphic image of $\mathbf{u}$, it follows that $\hat{r}^{-2 f} \hat{q}^{2 \sum_{(i, j) \in[\lambda]}(j-i)}=$ $\hat{r}^{-2 g} \hat{q}^{2 \sum_{(i, j) \in[\mu]}(j-i)}$; hence the result.

As the next example shows, Theorem 8.2 gives a sufficient but not the necessary condition for $B_{n}(\hat{q}, \hat{r})$ to be a semisimple algebra over $\kappa$; it can also be seen from the example that Theorem 8.5 gives a necessary but not sufficient condition for $\operatorname{Hom}_{B_{n}(\hat{q}, \hat{r})}\left(S^{\lambda}, S^{\mu}\right)$ to be non-zero.

Example 8.1 Let $n=3, \lambda=(1), \mu=(3), \kappa=\mathbb{Q}(\hat{q}, \hat{r})$, and suppose that $\hat{r}=-\hat{q}^{-3}$, where $\hat{q}$ is not a root of unity. Since $\hat{q}$ is not root of unity, the cell modules for $B_{3}(\hat{q}, \hat{r})$ corresponding to the partitions $(3),(2,1)$ and $(1,1,1)$ are absolutely irreducible (Theorem 3.43 of [8] together with Lemma 3.2 with $f=0$ ). On the other hand, if

$$
\mathfrak{s}=(\varnothing, \square, \square, \square) \in \mathfrak{T}_{n}(\lambda) \quad \text { and } \quad \mathfrak{t}=(\varnothing, \square, \square, \square, \square \square) \in \mathfrak{T}_{n}(\mu)
$$

then $\hat{P}(\mathfrak{s})=\left(1, \hat{q}^{2}, \hat{q}^{-2} \hat{r}^{-2}\right)=\left(1, \hat{q}^{2}, \hat{q}^{4}\right)$ and $\hat{P}(\mathfrak{t})=\left(1, \hat{q}^{2}, \hat{q}^{4}\right)$. Since $\hat{P}(\mathfrak{s})=\hat{P}(\mathfrak{t})$ whilst $\lambda \triangleright \mu$, the pair $\mathfrak{s}, \mathfrak{t}$ violates the hypotheses of Theorem 8.2. But we note by reference to the determinant of Gram matrix associated to $S^{\lambda}$ in Example 3.3 that $S^{\lambda}$ is absolutely irreducible and hence that $B_{3}(\hat{q}, \hat{r})$ remains semisimple over $\kappa$ (Theorems 4.3 and 4.4). 


\section{Brauer algebras}

The foregoing construction for the B-M-W algebras applies with minor modification to the Brauer algebras over an arbitrary field. We begin once more by considering Brauer algebras over a polynomial ring over $\mathbb{Z}$. Take $z$ to be an indeterminate over $\mathbb{Z}$; we write $R=\mathbb{Z}[z]$ and define the Brauer algebra $B_{n}(z)$ over $R$ as the associative unital $R$-algebra generated by the transpositions $s_{1}, s_{2}, \ldots, s_{n-1}$, together with elements $E_{1}, E_{2}, \ldots, E_{n-1}$, which satisfy the defining relations:

$$
\begin{array}{ll}
s_{i}^{2}=1 & \text { for } 1 \leq i<n ; \\
s_{i} s_{i+1} s_{i}=s_{i+1} s_{i} s_{i+1} & \text { for } 1 \leq i<n-1 ; \\
s_{i} s_{j}=s_{j} s_{i} & \text { for } 2 \leq|i-j| ; \\
E_{i}^{2}=z E_{i} & \text { for } 1 \leq i<n ; \\
s_{i} E_{j}=E_{j} s_{i} & \text { for } 2 \leq|i-j| ; \\
E_{i} E_{j}=E_{j} E_{i} & \text { for } 2 \leq|i-j| ; \\
E_{i} s_{i}=s_{i} E_{i}=E_{i} & \text { for } 1 \leq i<n ; \\
E_{i} s_{i \pm 1} s_{i}=s_{i \pm 1} s_{i} E_{i \pm 1}=E_{i} E_{i \pm 1} & \text { for } 1 \leq i, i \pm 1<n ; \\
E_{i} s_{i \pm 1} E_{i}=E_{i} E_{i \pm 1} E_{i}=E_{i} & \text { for } 1 \leq i, i \pm 1<n .
\end{array}
$$

Regard the group ring $R \mathfrak{S}_{n}$ as the subring of $B_{n}(z)$ generated by the transpositions $\left\{s_{i}=(i, i+1)\right.$ : for $\left.1 \leq i<n\right\}$. If $f$ is an integer, $0 \leq f \leq[n / 2]$, and $\lambda$ is a partition of $n-2 f$, define the elements

$$
x_{\lambda}=\sum_{w \in \mathfrak{S}_{\lambda}} w \quad \text { and } \quad m_{\lambda}=E_{1} E_{3} \cdots E_{2 f-1} x_{\lambda},
$$

where $\mathfrak{S}_{\lambda}$ is the row stabiliser in $\left\langle s_{2 f+1}, s_{2 f+2}, \ldots, s_{n-1}\right\rangle$ of the superstandard tableau $\mathfrak{t}^{\lambda} \in \operatorname{Std}_{n}(\lambda)$. Let $B_{n}^{\lambda}$ be the two sided ideal of $B_{n}(z)$ generated by $m_{\lambda}$ and write

$$
\check{B}_{n}^{\lambda}=\sum_{\mu \triangleright \lambda} B_{n}^{\mu}
$$

A cellular basis in terms of dangles has been given for the Brauer algebra in [5]. Replacing cellular bases for $\mathscr{H}_{n}\left(q^{2}\right)$ with cellular bases for $R \mathfrak{S}_{n}$, the process used to construct cellular bases the B-M-W algebras in [4] will produce also cellular bases for $B_{n}(z)$ as follows.

If $f$ is an integer, $0 \leq f \leq[n / 2]$, and $\lambda$ a partition of $n-2 f$, then $\mathcal{I}_{n}(\lambda)$ retains the meaning assigned in (3.3).

Theorem 9.1 The algebra $B_{n}(z)$ is freely generated as an $R-$ module by the collection

$$
\left\{\begin{array}{l|l}
(d(\mathfrak{s}) v)^{-1} m_{\lambda} d(\mathfrak{t}) u \mid \begin{array}{c}
(\mathfrak{s}, v),(\mathfrak{t}, u) \in \mathcal{I}_{n}(\lambda) \text { for } \lambda \text { a partition } \\
\text { of } n-2 f \text { and } 0 \leq f \leq[n / 2]
\end{array}
\end{array}\right\}
$$


Moreover, the following statements hold.

1. The R-linear map determined by

$$
(d(\mathfrak{s}) v)^{-1} m_{\lambda} d(\mathfrak{t}) u \mapsto(d(\mathfrak{t}) u)^{-1} m_{\lambda} d(\mathfrak{s}) v
$$

is an algebra anti-involution of $B_{n}(z)$.

2. Suppose that $b \in B_{n}(z)$ and let $f$ be an integer, $0 \leq f \leq[n / 2]$. If $\lambda$ is a partition of $n-2 f$ and $(\mathfrak{t}, u) \in \mathcal{I}_{n}(\lambda)$, then there exist $a_{(\mathfrak{u}, w)} \in R$, for $(\mathfrak{u}, w) \in \mathcal{I}_{n}(\lambda)$, such that for all $(\mathfrak{s}, v) \in \mathcal{I}_{n}(\lambda)$,

$$
(d(\mathfrak{s}) v)^{-1} m_{\lambda} d(\mathfrak{t}) u b \equiv \sum_{(\mathfrak{u}, w)} a_{(\mathfrak{u}, w)}(d(\mathfrak{s}) v)^{-1} m_{\lambda} d(\mathfrak{u}) w \quad \bmod \check{B}_{n}^{\lambda} .
$$

As a consequence of the above theorem, $\check{B}_{n}^{\lambda}$ is the $R$-module freely generated by

$$
\left\{(d(\mathfrak{s}) v)^{-1} m_{\mu} d(\mathfrak{t}) u:(\mathfrak{s}, v),(\mathfrak{t}, u) \in \mathcal{I}_{n}(\mu), \text { for } \mu \triangleright \lambda\right\} .
$$

If $f$ is an integer, $0 \leq f \leq[n / 2]$, and $\lambda$ is a partition of $n-2 f$, the cell module $S^{\lambda}$ is defined to be the $R$-module freely generated by

$$
\left\{m_{\lambda} d(\mathfrak{t}) u+\check{B}_{n}^{\lambda} \mid(\mathfrak{t}, u) \in \mathcal{I}_{n}(\lambda)\right\}
$$

with right $B_{n}(z)$ action

$$
\left(m_{\lambda} d(\mathfrak{t}) u\right) b+\check{B}_{n}^{\lambda}=\sum_{(\mathfrak{u}, w)} a_{(\mathfrak{u}, w)} m_{\lambda} d(\mathfrak{u}) w+\check{B}_{n}^{\lambda} \quad \text { for } b \in B_{n}(z),
$$

where the coefficients $a_{(\mathfrak{u}, w)} \in R$, for $(\mathfrak{u}, w)$ in $\mathcal{I}_{n}(\lambda)$, are determined by the expression (9.1).

The construction of cellular algebras [5] equips the $B_{n}(z)$-module $S^{\lambda}$ with a symmetric associative bilinear form (compare (3.6) above). Following is the counterpart to Example 3.3, stated for reference in Sect. 11.

Example 9.1 Let $n=3$ and $\lambda=(1)$ so that $\check{B}_{n}^{\lambda}=(0)$ and $m_{\lambda}=E_{1}$. We order the basis (9.2) for the module $S^{\lambda}$ as $\mathbf{v}_{1}=E_{1}, \mathbf{v}_{2}=E_{1} s_{2}$ and $\mathbf{v}_{3}=E_{1} s_{2} s_{1}$ and, with respect to this ordered basis, the Gram matrix $\left\langle\mathbf{v}_{i}, \mathbf{v}_{j}\right\rangle$ of the bilinear form on the $B_{n}(z)$-module $S^{\lambda}$ is

$$
\left[\begin{array}{lll}
z & 1 & 1 \\
1 & z & 1 \\
1 & 1 & z
\end{array}\right]
$$

The determinant of the Gram matrix given above is

$$
(z-1)^{2}(z+2) \text {. }
$$

By Theorem 2.3 of [12], the Bratteli diagram associated with $B_{n}(z)$ is identical to the Bratteli diagram for $B_{n}(q, r)$. Thus $\mu \rightarrow \lambda$ retains the meaning assigned in Sect. 5. 
Let $f$ be an integer, $0 \leq f \leq[n / 2]$, and $\lambda$ be a partition of $n-2 f$ with $t$ removable nodes and $(p-t)$ addable nodes. Suppose that $\mu^{(1)} \triangleright \mu^{(2)} \triangleright \cdots \triangleright \mu^{(p)}$ is the ordering of $\{\mu: \mu \rightarrow \lambda\}$ by dominance order on partitions. If $1 \leq k \leq t$, define

$$
y_{\mu^{(k)}}^{\lambda}=m_{\lambda} d(\mathfrak{s})+\check{B}_{n}^{\lambda} \quad \text { where }\left.\mathfrak{s}\right|_{n-1}=\mathfrak{t}^{\mu^{(k)}} \in \operatorname{Std}_{n-1}\left(\mu^{(k)}\right)
$$

and, if $t<k \leq p$ define $w_{k}$ by (5.20) and, by analogy with (5.21), write

$$
y_{\mu^{(k)}}^{\lambda}=E_{2 f-1} w_{k}^{-1} m_{\mu^{(k)}}+\check{B}_{n}^{\lambda} .
$$

Given the elements $y_{\mu}^{\lambda}$ in $S^{\lambda}$ for each partition $\mu \rightarrow \lambda$, define $N^{\mu}$ to be the $B_{n-1}(z)-$ submodule of $S^{\lambda}$ generated by

$$
\left\{y_{v}^{\lambda}: v \rightarrow \lambda \text { and } v \unrhd \mu\right\}
$$

and let $\check{N}^{\mu}$ be the $B_{n-1}(z)-$ submodule of $S^{\lambda}$ generated by

$$
\left\{y_{v}^{\lambda}: \nu \rightarrow \lambda \text { and } v \triangleright \mu\right\} .
$$

Theorem 5.9 and the construction given for the B-M-W algebras in Sect. 6 have analogues in the context of $B_{n}(z)$. Thus the cell module (9.2) has a basis over $R$,

$$
\left\{m_{\mathfrak{t}}=m_{\lambda} b_{\mathfrak{t}}+\check{B}_{n}^{\lambda}: \mathfrak{t} \in \mathfrak{T}_{n}(\lambda)\right\}
$$

indexed by the paths $\mathfrak{T}_{n}(\lambda)$ of shape $\lambda$ in the Bratteli diagram associated with $B_{n}(z)$, and defined in the same manner as the basis (6.5).

\section{Jucys-Murphy operators for the Brauer algebras}

Define the operators $L_{i}$, for $i=1, \ldots, n$, in $B_{n}(z)$ by $L_{1}=0$ and

$$
L_{i}=s_{i-1}-E_{i-1}+s_{i-1} L_{i-1} s_{i-1} \quad \text { for } 1<i \leq n .
$$

Remark 10.1 The elements $L_{i}$ as defined above bear an obvious analogy with the elements $\tilde{D}_{i}$ defined in Sect. 2.2; thus we refer to the elements $L_{i}$ as the "JucysMurphy operators" in $B_{n}(z)$.

In [10], M. Nazarov made use of operators $x_{i}$ with are related to the $L_{i}$ defined above by $x_{i}=\frac{z-1}{2}+L_{i}$. Since the difference of $L_{i}$ and the $x_{i}$ of [10] is a scalar multiple of the identity, we derive the next statement from results in Sect. 2 of [10].

Proposition 10.1 Let $i$ and $k$ be integers, $1 \leq i<n$ and $1 \leq k \leq n$.

1. $s_{i}$ and $L_{k}$ commute if $i \neq k-1, k$.

2. $L_{i}$ and $L_{k}$ commute.

3. $s_{i}$ commutes with $L_{i}+L_{i+1}$.

4. $L_{2}+L_{3}+\cdots+L_{n}$ belongs to the centre of $B_{n}(z)$. 
For integers $j, k$ with $1 \leq j, k \leq n$, we define the elements $L_{k}^{(j)}$ by $L_{1}^{(j)}=0$ and

$$
L_{k+1}^{(j)}=s_{j+k-1}-E_{j+k-1}+s_{j+k-1} L_{k}^{(j)} s_{j+k-1}, \quad \text { for } k \geq 1 .
$$

In particular, $L_{k}^{(1)}=L_{k}$, for $k=1, \ldots, n$, are the Jucys-Murphy elements for $B_{n}(z)$.

The objective now is to show that $m_{\mathfrak{t}^{\lambda}}$ is a common eigenvector for the action of the Jucys-Murphy elements $L_{k}$ on the cell module $S^{\lambda}$.

Proposition 10.2 Let $i, k$ be integers with $1 \leq i \leq n$ and $1<k \leq n$. Then

$$
E_{i} L_{k}^{(i)}= \begin{cases}(1-z) E_{i} & \text { if } k=2 \\ 0 & \text { if } k=3 \\ E_{i} L_{k-2}^{(i+2)} & \text { if } k \geq 4\end{cases}
$$

Proof If $k=2$ then $E_{i} L_{k}^{(i)}=E_{i}\left(s_{i}-E_{i}\right)=(1-z) E_{i}$. For $k=3$ we have

$$
\begin{aligned}
E_{i} L_{3}^{(i)} & =E_{i}\left(s_{i+1}-E_{i+1}+s_{i+1} s_{i} s_{i+1}-s_{i+1} E_{i} s_{i+1}\right) \\
& =E_{i}\left(s_{i+1}-E_{i+1}\right)+E_{i}\left(E_{i+1} s_{i+1}-s_{i+1}\right)=0 .
\end{aligned}
$$

If $k=4$ then,

$$
\begin{aligned}
E_{i} L_{4}^{(i)} & =E_{i}\left(s_{i+2}-E_{i+2}\right)+s_{i+2} E_{i} L_{3}^{(i)} s_{i+2} \\
& =E_{i}\left(s_{i+2}-E_{i+2}\right)=E_{i} L_{2}^{(i+2)},
\end{aligned}
$$

and when $k>4$,

$$
\begin{aligned}
E_{i} L_{k}^{(i)} & =E_{i}\left(s_{i+k-2}-E_{i+k-2}\right)+s_{i+k-2} E_{i} L_{k-1}^{(i)} s_{i+k-2} \\
& =E_{i}\left(s_{i+k-2}-E_{i+k-2}\right)+s_{i+k-2} E_{i} L_{k-3}^{(i+2)} s_{i+k-2} \\
& =E_{i}\left(s_{i+k-2}-E_{i+k-2}+s_{i+k-2} L_{k-3}^{(i+2)} s_{i+k-2}\right)=E_{i} L_{k-2}^{(i+2)}
\end{aligned}
$$

by induction.

Corollary 10.3 Let $f, k$ be integers, $0<f \leq[n / 2]$ and $1 \leq k \leq n$. Then

$$
E_{1} E_{3} \cdots E_{2 f-1} L_{k}= \begin{cases}0, & \text { if } k \text { is odd }, 1 \leq k \leq 2 f+1 \\ (1-z) E_{1} E_{3} \cdots E_{2 f-1}, & \text { if } k \text { is even, } 1<k \leq 2 f \\ E_{1} E_{3} \cdots E_{2 f-1} L_{k-2 f}^{(2 f+1)}, & \text { if } 2 f+1<k \leq n\end{cases}
$$

Proof If $k$ is odd, $1<k \leq 2 f+1$, then by Proposition 10.2,

$$
\begin{gathered}
E_{1} E_{3} \cdots E_{k} L_{k}=E_{1} E_{3} \cdots E_{k} L_{k}^{(1)}=E_{1} E_{3} \cdots E_{k} L_{k-2}^{(3)}=\cdots \\
\cdots=E_{1} E_{3} \cdots E_{k} L_{1}^{(k)}=0
\end{gathered}
$$


Since $E_{k+2} E_{k+3} \cdots E_{2 f-1}$ commutes with $L_{k}$, the first case follows. If $k$ is even and $1<k \leq 2 f$, then the relations $E_{i} s_{i}=E_{i}$ and $E_{i}^{2}=z E_{i}$, together with (10.1), show that

$$
\begin{aligned}
E_{1} E_{3} \cdots E_{2 f-1} L_{k} & =E_{1} E_{3} \cdots E_{2 f-1}\left(s_{k-1}-E_{k-1}+s_{k-1} L_{k-1} s_{k}\right) \\
& =(1-z) E_{1} E_{3} \cdots E_{2 f-1}+E_{1} E_{3} \cdots E_{2 f-1} L_{k-1} s_{k-1} \\
& =(1-z) E_{1} E_{3} \cdots E_{2 f-1} .
\end{aligned}
$$

The final case follows in a similar manner.

Let $f$ be an integer, $0 \leq f \leq[n / 2]$, and $\lambda$ be a partition of $n-2 f$. For each path $\mathfrak{t} \in \mathfrak{T}_{n}(\lambda)$, define the polynomial

$$
P_{\mathfrak{t}}(k)= \begin{cases}j-i & \text { if }\left[\lambda^{(k)}\right]=\left[\lambda^{(k-1)}\right] \cup\{(i, j)\} \\ i-j+1-z & \text { if }\left[\lambda^{(k)}\right]=\left[\lambda^{(k-1)}\right] \backslash\{(i, j)\} .\end{cases}
$$

The proof of the next statement is identical to the proof of Proposition 7.5 given above; for the proof of Proposition 10.5, we refer to the proof of Proposition 7.6.

Proposition 10.4 If $\lambda$ is a partition of $n$ and $k$ is an integer with $1 \leq k \leq n$, then $m_{\mathfrak{t}^{\lambda}} L_{k}=P_{\mathfrak{t}^{\lambda}}(k) m_{\mathfrak{t}^{\lambda}}$.

Proposition 10.5 Let $f$ be an integer, $0<f \leq[n / 2]$, and $\lambda$ be a partition of $n-2 f$. Then $m_{\mathfrak{t}^{\lambda}} L_{k}=P_{\mathfrak{t}^{\lambda}}(k) m_{\mathfrak{t}^{\lambda}}$.

Proposition 10.6 Let $f$ be an integer, $0 \leq f \leq[n / 2]$, and $\lambda$ be a partition of $n-2 f$. Then there exists an invariant $\alpha \in R$ such that $L_{2}+L_{3}+\cdots+L_{n}$ acts on $S^{\lambda}$ as a scalar multiple by $\alpha$ of the identity.

Proof As in the proof of Proposition 7.7, we obtain $\alpha=\sum_{k=2}^{n} P_{\mathfrak{t}^{\lambda}}(k)$.

Theorem 10.7 Let $f$ be an integer $0 \leq f \leq[n / 2]$ and $\lambda$ be a partition of $n-2 f$. If $\mathfrak{t} \in \mathfrak{T}_{n}(\lambda)$, then there exist $a_{\mathfrak{v}} \in R$, for $\mathfrak{v} \in \mathfrak{T}_{n}(\lambda)$ with $\mathfrak{v} \triangleright \mathfrak{t}$, such that

$$
m_{\mathfrak{t}} L_{k}=P_{\mathfrak{t}}(k) m_{\mathfrak{t}}+\sum_{\substack{\mathfrak{v} \in \mathfrak{T}_{n}(\lambda) \\ \mathfrak{v} \triangleright \mathfrak{t}}} a_{\mathfrak{v}} m_{\mathfrak{v}}
$$

Proof By repeating word for word the argument given in the proof of Theorem 7.8, we show that the statement holds true when $1 \leq k<n$.

That $L_{n}$ acts triangularly on $S^{\lambda}$, can then be observed using Proposition 10.6:

$$
m_{\mathfrak{t}} L_{n}=\sum_{k=1}^{n} P_{\mathfrak{t}}(k) m_{\mathfrak{t}}-m_{\mathfrak{t}}\left(L_{2}+L_{3}+\cdots+L_{n-1}\right) .
$$

Thus the generalised eigenvalue for $L_{n}$ acting on $m_{\mathfrak{t}}$ is $P_{\mathfrak{t}}(n)$. 


\section{Semisimplicity criteria for Brauer algebras}

Below are analogues for the Brauer algebras of the results of Sect. 8. Let $\kappa$ be a field and take $\hat{z} \in \kappa$. Then $z \mapsto \hat{z}$ determines a homomorphism $R \rightarrow \kappa$, giving $\kappa$ an $R-$ module structure. A Brauer algebra over $\kappa$ is a specialisation $B_{n}(\hat{z})=B_{n}(z) \otimes_{R} \kappa$. For $\mathfrak{t} \in \mathfrak{T}_{n}(\lambda)$ and $k=1, \ldots, n$, let $\hat{P}_{\mathfrak{t}}(k)$ denote the evaluation of the monomial $P_{\mathfrak{t}}(k)$ at $\hat{z}$,

$$
\hat{P}_{\mathfrak{t}}(k)= \begin{cases}j-i & \text { if }\left[\lambda^{(k)}\right]=\left[\lambda^{(k-1)}\right] \cup\{(i, j)\} \\ i-j+1-\hat{z} & \text { if }\left[\lambda^{(k)}\right]=\left[\lambda^{(k-1)}\right] \backslash\{(i, j)\},\end{cases}
$$

and as previously, define the ordered $n$-tuple $\hat{P}(\mathfrak{t})=\left(\hat{P}_{\mathfrak{t}}(1), \ldots, \hat{P}_{\mathfrak{t}}(n)\right)$. The operators $L_{i}$ provide conditions necessary for the existence of a homomorphic image of one cell module for $B_{n}(\hat{z})$ in another cell module for $B_{n}(\hat{z})$.

Theorem 11.1 Let $\kappa$ be a field. Suppose that for each pair of partitions $\lambda$ of $n-2 f$ and $\mu$ of $n-2 f^{\prime}$, for integers $f, f^{\prime}$ with $0 \leq f, f^{\prime} \leq[n / 2]$, and for each pair of partitions $\mathfrak{s} \in \mathfrak{T}_{n}(\lambda)$ and $\mathfrak{t} \in \mathfrak{T}_{n}(\mu)$, the conditions $\lambda \unrhd \mu$ and $\hat{P}(\mathfrak{s})=\hat{P}(\mathfrak{t})$ together imply that $\lambda=\mu$. Then $B_{n}(\hat{z})$ is a semisimple algebra over $\kappa$.

By an analogous statement to Lemma 8.3, the Jucys-Murphy elements do in fact distinguish between the cell modules of $B_{n}(z)$ in Theorem 11.1.

The results of this section can be used to derive the next statement which is Theorem 3.3 of [3]. As in Theorem 8.5, the statement may be generalised to the setting where $|\lambda|>|\mu|$.

Theorem 11.2 Let $\lambda$ be a partition of $n$ and $\mu$ be a partition of $n-2 f$, where $f>0$. If $\operatorname{Hom}_{B_{n}(\hat{z})}\left(S^{\lambda}, S^{\mu}\right) \neq 0$, then

$$
\sum_{(i, j) \in[\lambda]}(j-i)-\sum_{(i, j) \in[\mu]}(j-i)=f(1-\hat{z}) .
$$

Proof Suppose that $\mathbf{u} \in S^{\lambda}, \mathbf{v} \in S^{\mu}$ are non-zero and that $\mathbf{u} \mapsto \mathbf{v}$ under some element in $\operatorname{Hom}_{B_{n}(\hat{z})}\left(S^{\lambda}, S^{\mu}\right)$. Then, using Proposition 10.6,

$$
\sum_{i=1}^{n} \mathbf{u} L_{i}=\sum_{(i, j) \in[\lambda]}(j-i) \mathbf{u}
$$

while

$$
\sum_{i=1}^{n} \mathbf{v} L_{i}=f(1-\hat{z}) \mathbf{v}+\sum_{(i, j) \in[\mu]}(j-i) \mathbf{v}
$$

Since $\mathbf{v}$ is the homomorphic image of $\mathbf{u}$, it follows that

$$
\sum_{(i, j) \in[\lambda]}(j-i)=f(1-\hat{z})+\sum_{(i, j) \in[\mu]}(j-i) .
$$


Hence the result.

Theorem 11.1 gives a sufficient but not the necessary condition for $B_{n}(\hat{z})$ to be a semisimple algebra over $\kappa$. Necessary and sufficient conditions on the semisimplicity of $B_{n}(\hat{z})$ have been given by H. Rui in [11].

Example 11.1 Let $\kappa=\mathbb{Q}$ and $\hat{z}=4$. Take $n=3, \lambda=(1)$ and $\mu=(1,1,1)$. In characteristic zero the cell modules corresponding to the partitions $(3),(2,1)$ and $(1,1,1)$ are absolutely irreducible. But, taking

$$
\mathfrak{t}=(\varnothing, \square, \square, \square) \in \mathfrak{T}_{n}(\lambda) \quad \text { and } \quad \mathfrak{u}=(\varnothing, \square, \square, \square) \in \mathfrak{T}_{n}(\mu),
$$

then

$$
\hat{P}(\mathfrak{t})=(0,-1,2-\hat{z})=(0,-1,-2) \quad \text { and } \quad \hat{P}(\mathfrak{u})=(0,-1,-2) .
$$

Since $\hat{P}(\mathfrak{t})=\hat{P}(\mathfrak{u})$ whilst $\lambda \triangleright \mu$, the pair $\mathfrak{t}, \mathfrak{u}$ violates the hypotheses of Theorem 11.1. However, by reference to the determinant of Gram matrix associated to $S^{\lambda}$ in Example 9.1, it follows that $S^{\lambda}$ is absolutely irreducible and hence that $B_{3}(\hat{z})$ remains semisimple by appeal to appropriate analogues of Theorems 4.3 and 4.4.

\section{Conjectures}

Define a sequence of polynomials $\left(p_{i}(z) \mid i=1,2, \ldots,\right)$ by $p_{1}(z)=(z+2)(z-1)$ and

$$
p_{i}(z)= \begin{cases}(z+2 i)(z-i)(z+i-2) p_{i-1}(z) & \text { if } i \text { is odd } \\ (z+2 i)(z-i) p_{i-1}(z) & \text { if } i \text { is even }\end{cases}
$$

Conjecture 12.1 For $\kappa$ a field, $\hat{z} \in \kappa$ and an algebra over $\kappa$, with $n \geq 2$, the following statements hold:

(i) If $n=2 k+1$, then the bilinear form on the $B_{n}(\hat{z})$-module $S^{(1)}$ determined by (3.6) is non-degenerate if and only if $p_{k}(\hat{z}) \neq 0$.

(ii) If $n=2 k$, then the bilinear form on the $B_{n}(\hat{z})-$ module $S^{\varnothing}$ determined by (3.6) is non-degenerate if and only if $\hat{z} \neq 0$ and $p_{k}(\hat{z}) \neq 0$.

Conjecture 12.2 For $\kappa$ a field, $\hat{z} \in \kappa$ and an algebra over $\kappa$, with $n \geq 2$, the following statements hold:

(i) If $n=2 k+1$, then $B_{n}(\hat{z})$ is semisimple and only if $\kappa \mathfrak{S}_{n}$ is semisimple and $p_{2 k-1}(\hat{z}) \neq 0$.

(ii) If $n=2 k$, then $B_{n}(\hat{z})$ is semisimple and only if $\kappa \mathfrak{S}_{n}$ is semisimple, $\hat{z} \neq 0$ and $p_{2 k-2}(\hat{z}) \neq 0$. 


\section{References}

1. Birman, J., Wenzl, H.: Braids, link polynomials and a new algebra. Trans. Am. Math. Soc. 313(1), 249-273 (1989)

2. Dipper, R., James, G.: Representations of Hecke algebras of general linear groups. Proc. LMS 52(3), 20-52 (1986)

3. Doran, W., Wales, D., Hanlon, P.: On the semisimplicity of the Brauer centralizer algebras. J. Algebra 211, 647-685 (1999)

4. Enyang, J.: Cellular bases for the Brauer Birman-Murakami-Wenzl algebras. J. Algebra 281, 413449 (2004)

5. Graham, J., Lehrer, G.: Cellular algebras. Invent. Math. 123(1), 1-34 (1996)

6. Leduc, R., Ram, A.: A Ribbon Hopf algebra approach to the irreducible representations of centralizer algebras: the Brauer. Birman-Wenzl, and Type A Iwahori-Hecke algebras. Adv. Math. 125(1), 1-94 (1997)

7. Macdonald, I.: Symmetric Functions and Hall Polynomials, 2nd edn. Oxford Mathematical Monographs. Clarendon Press, Oxford (1995)

8. Mathas, A.: Iwahori-Hecke Algebras and Schur Algebras of the Symmetric Group. University Lecture Series, vol. 15, American Mathematical Society, Providence (1999)

9. Murphy, E.: The representations of Hecke algebras of type $A_{n}$. J. Algebra 173(1), 97-121 (1995)

10. Nazarov, M.: Young's orthogonal form for Brauer's centralizer algebra. J. Algebra 182(3), 664-693 (1996)

11. Rui, H.: A criterion on the semisimple Brauer algebras. J. Comb. Theory Ser. A 111, $78-88$ (2005)

12. Wenzl, H.: Quantum groups and subfactors of type B, C, and D. Commun. Math. Phys. 133(2), 383342 (1990)

13. Xi, C.C.: On the quasi-heredity of Birman-Wenzl algebras. Adv. Math. 154(2), 280-298 (2000) 